

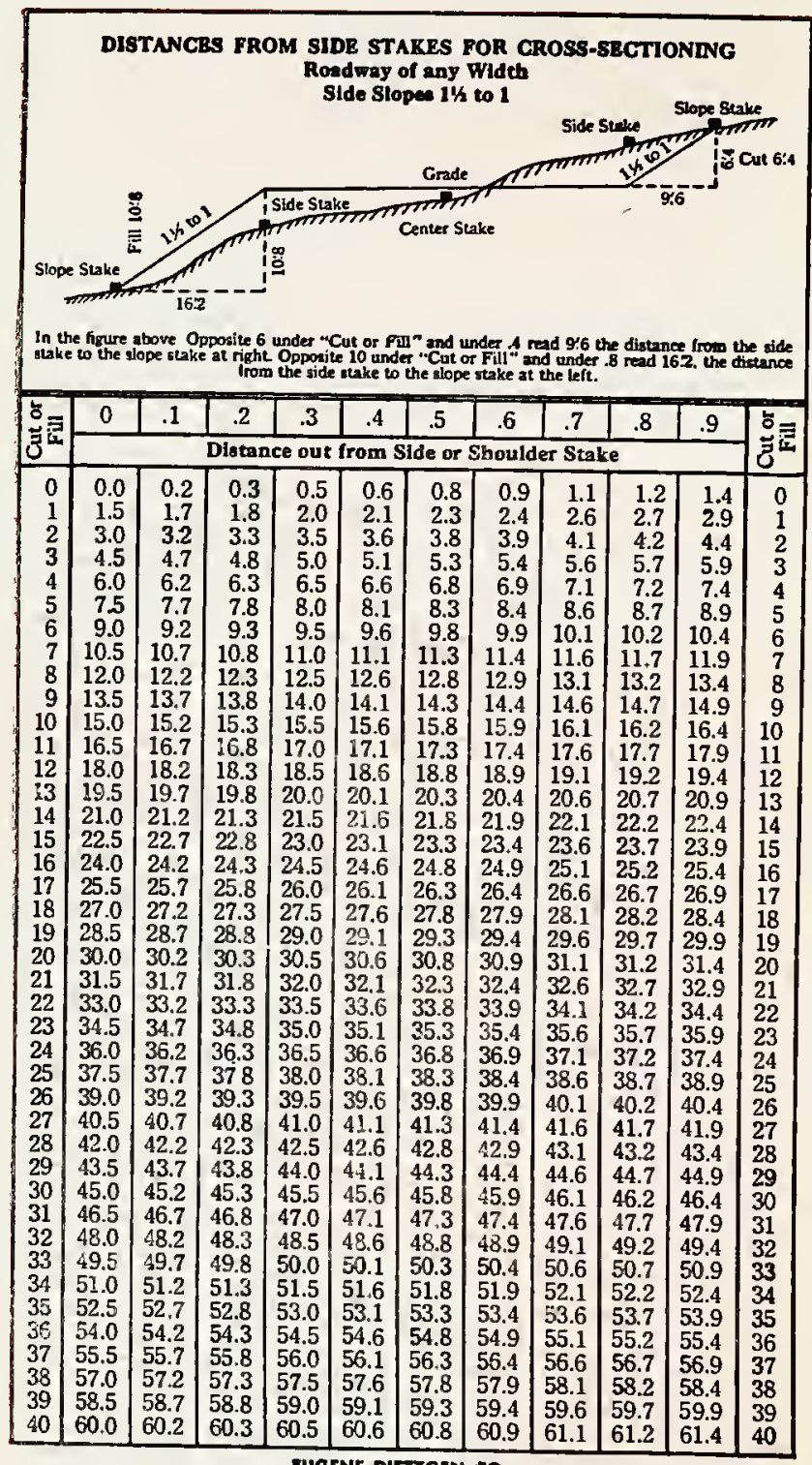

IUOENE DIETIGEN CO. 


\section{Property of}

Address

Phone

This Field Book contains special paper which is impregnated with resin to make it substantially stronger as weil as water resistant. Your field notes will come out sharp and clear even when the page is wet. 


\section{INDEX PAGE}

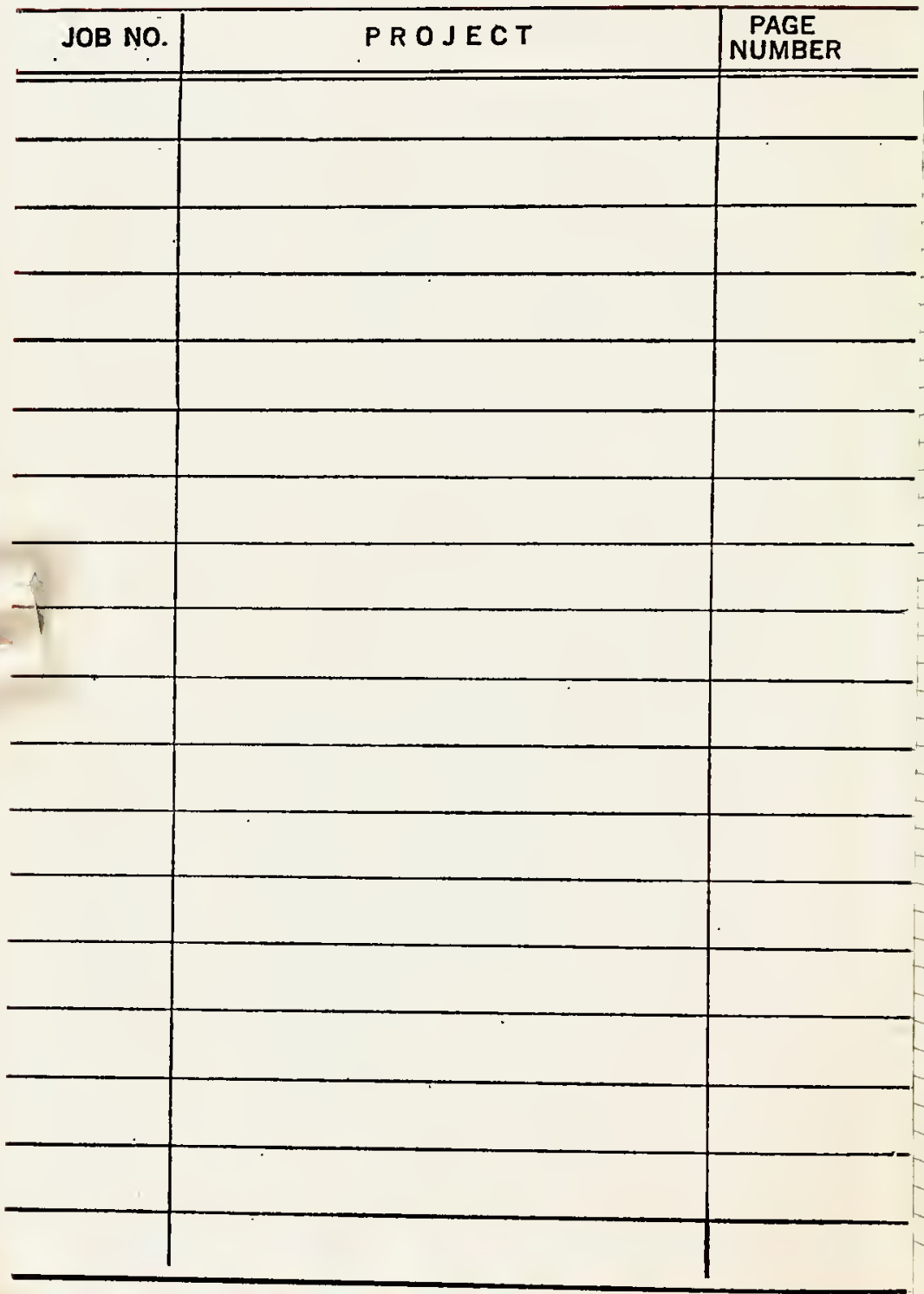




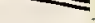
$1+n+1$
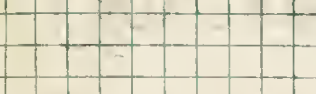


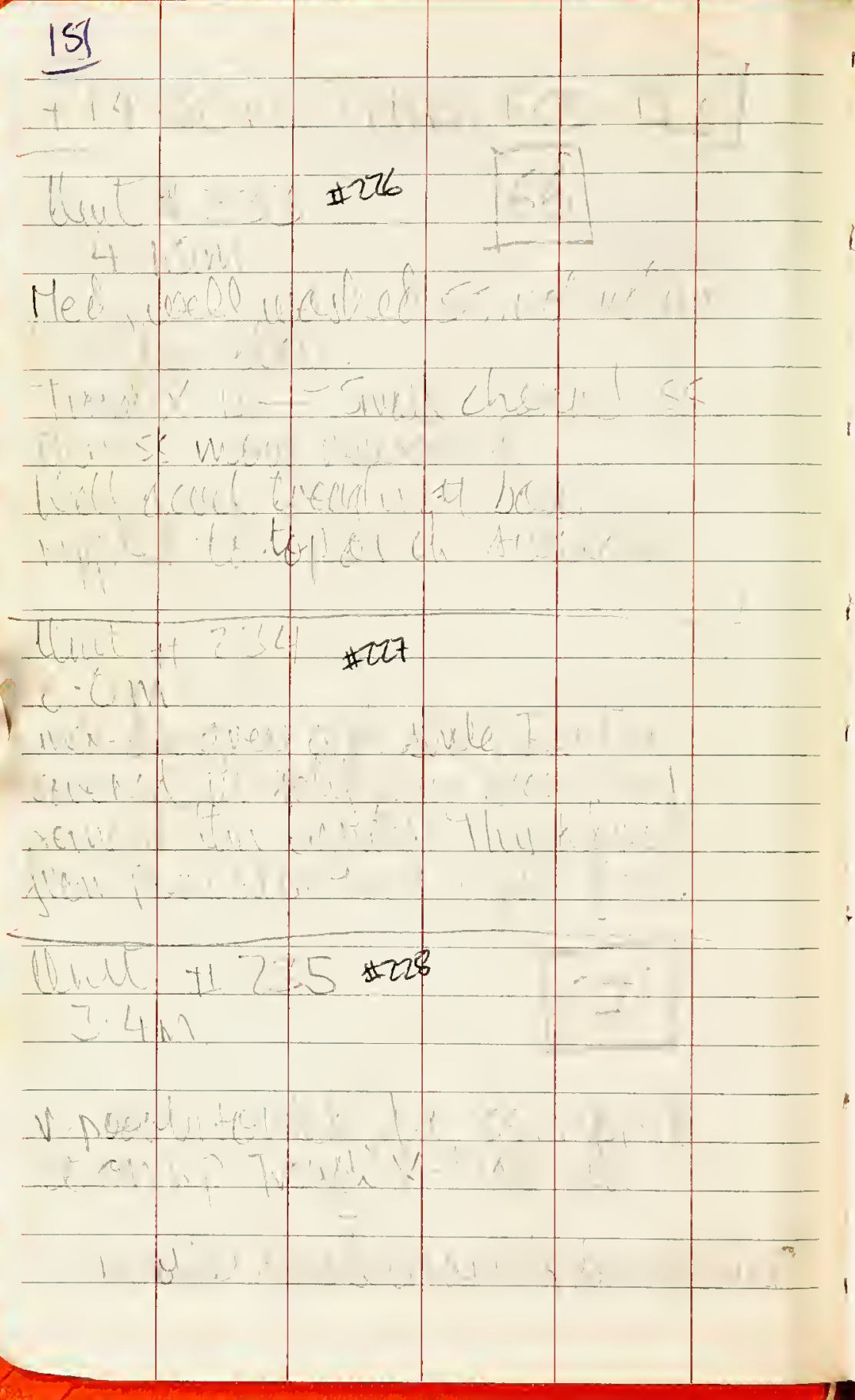


152

C.

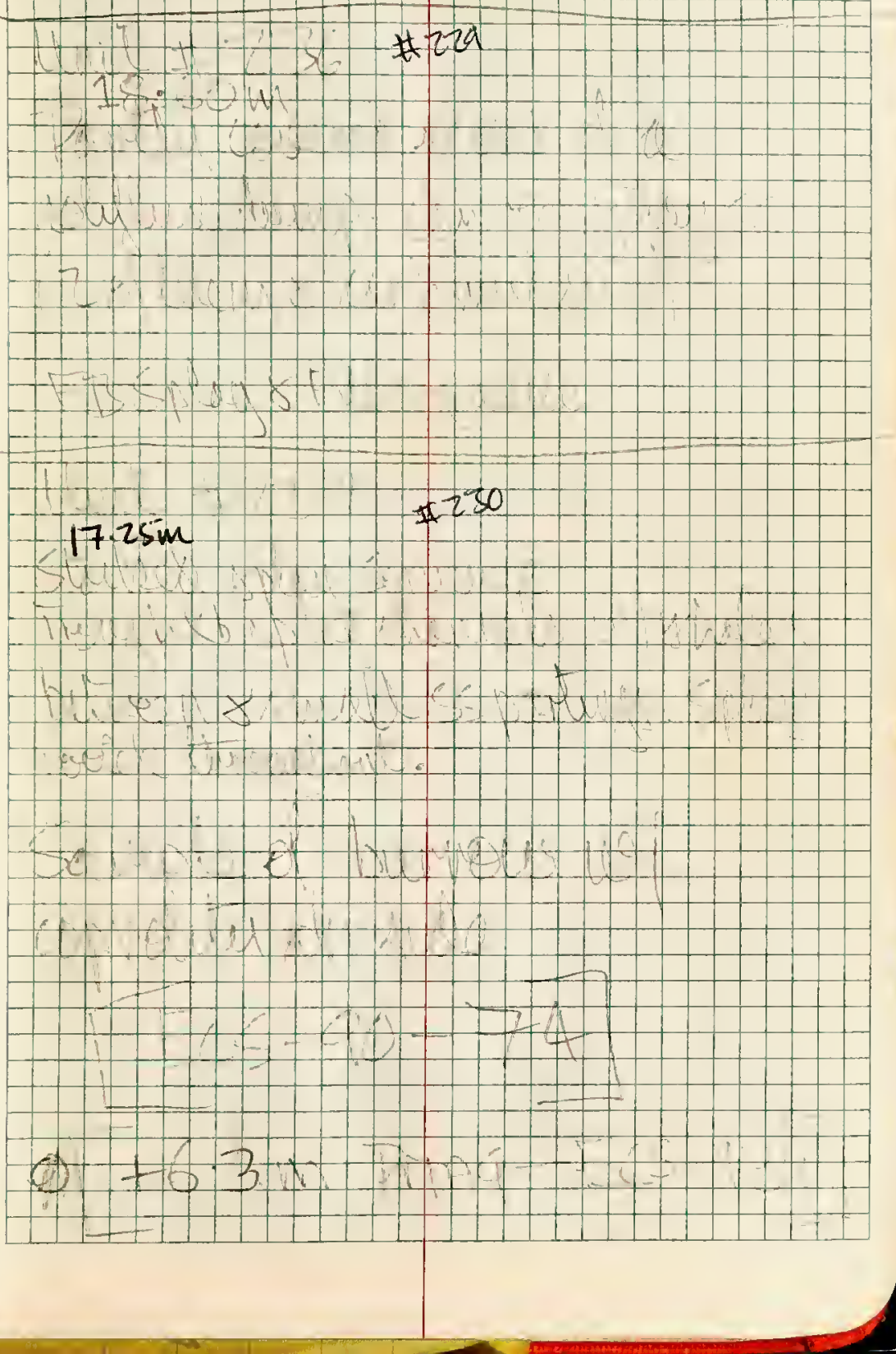


154

Ute ti 
$x+\cos 4$

Tilis

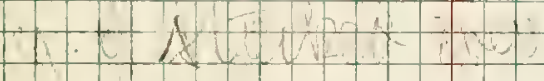

$-\frac{x}{x}+1$

-1ein

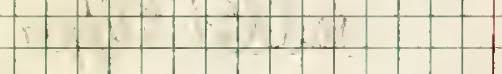

$y+$ 


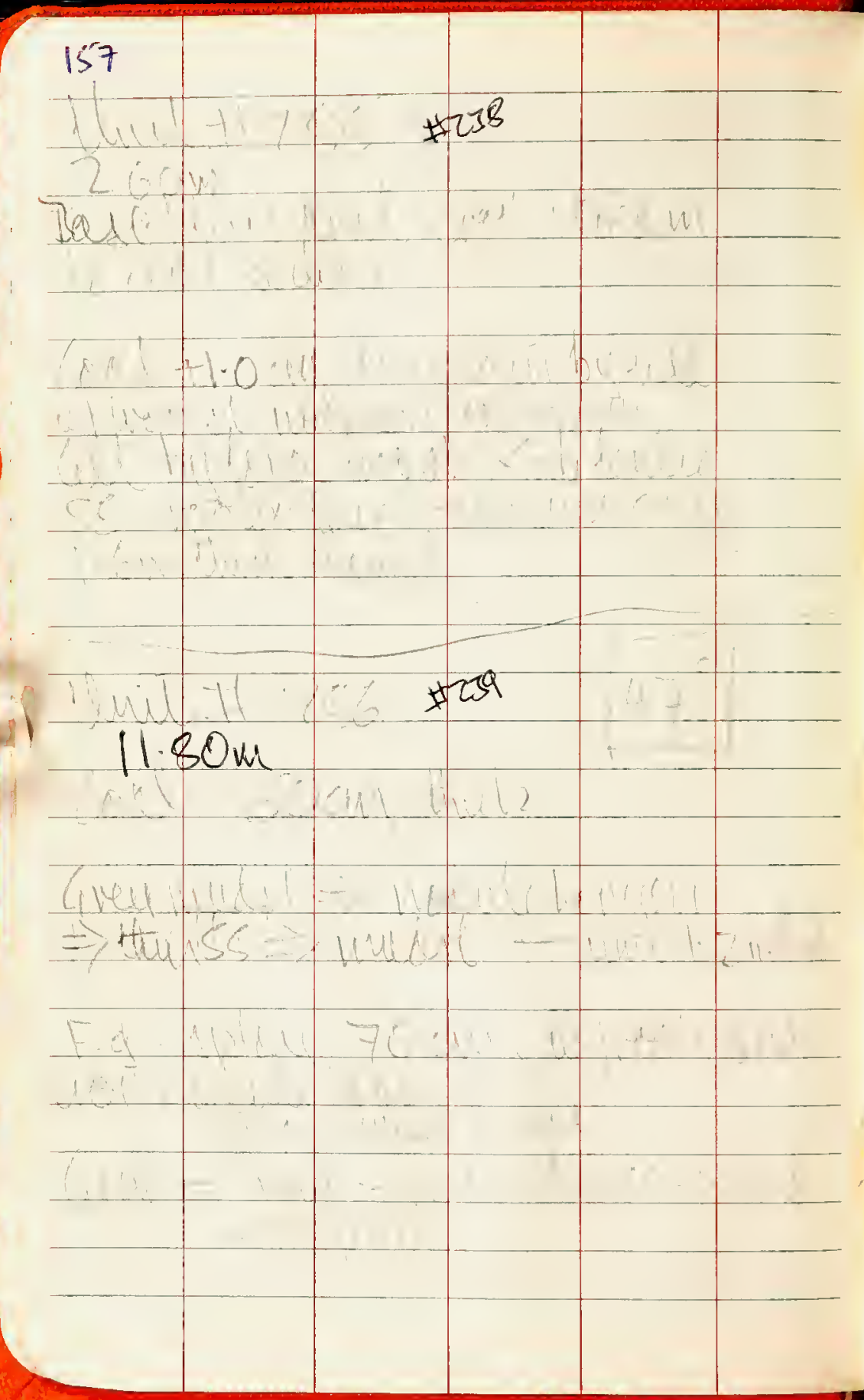



$+(1+3+4+1$

(1) (5)

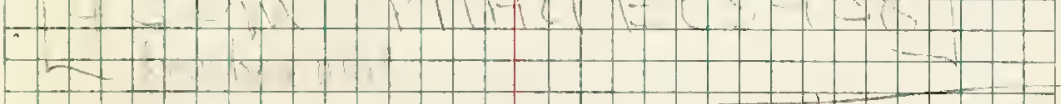
\begin{tabular}{|l|l|l|l|l|l|l|} 
& & & & & & \\
\hline
\end{tabular}

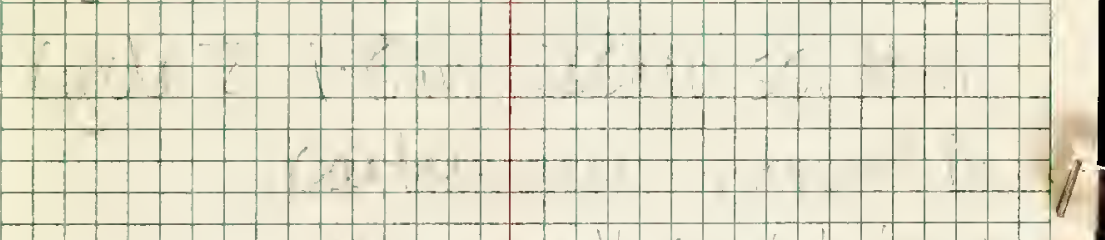

$+1$

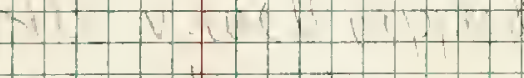

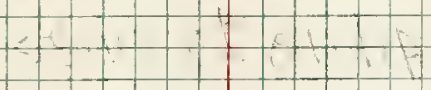
$+\div, \frac{1}{1}, \ldots$

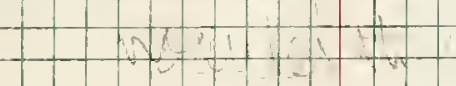

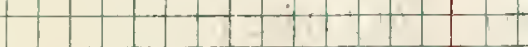




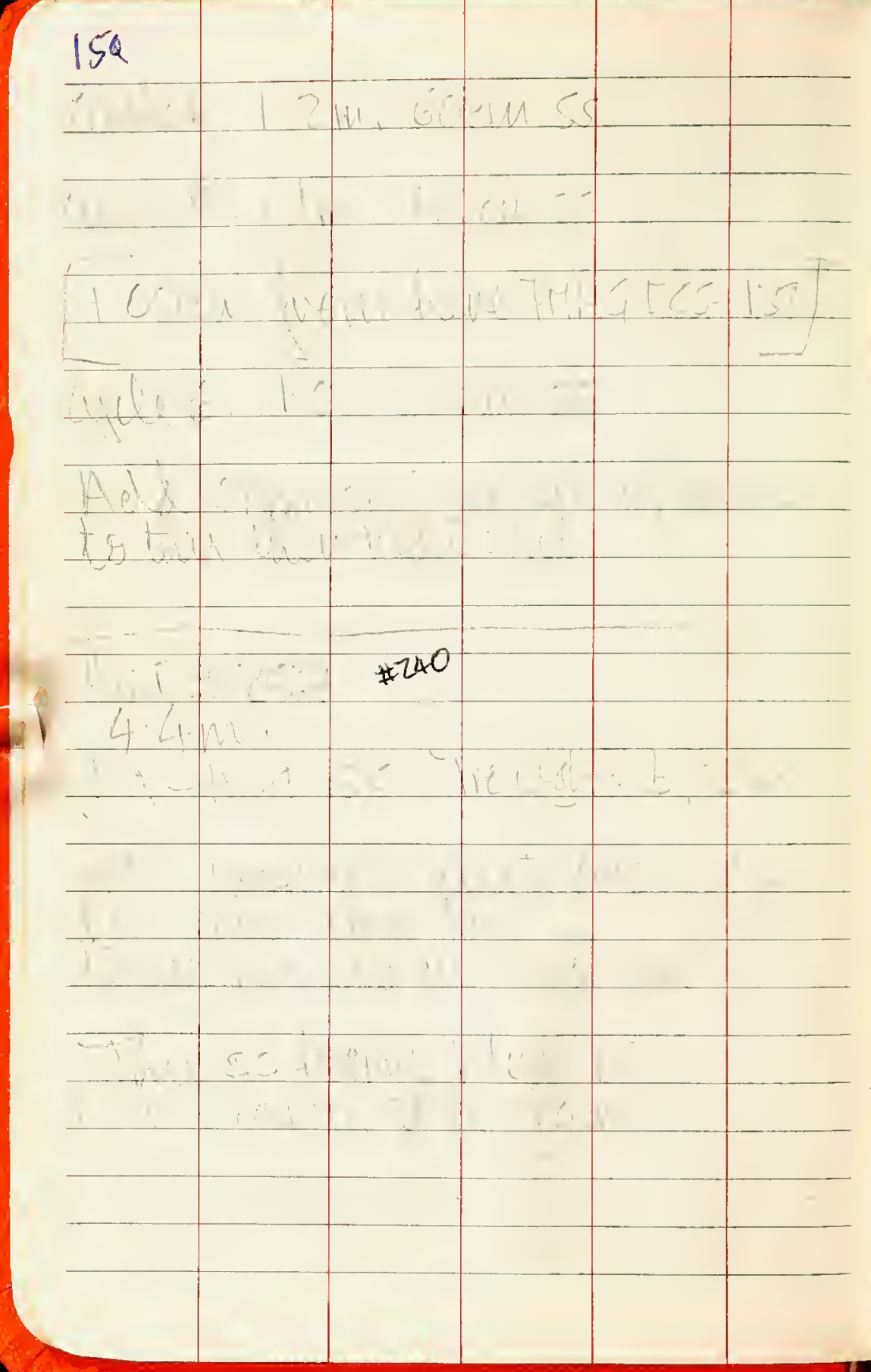


160

$$
20 \mathrm{~m}
$$

\#24) 


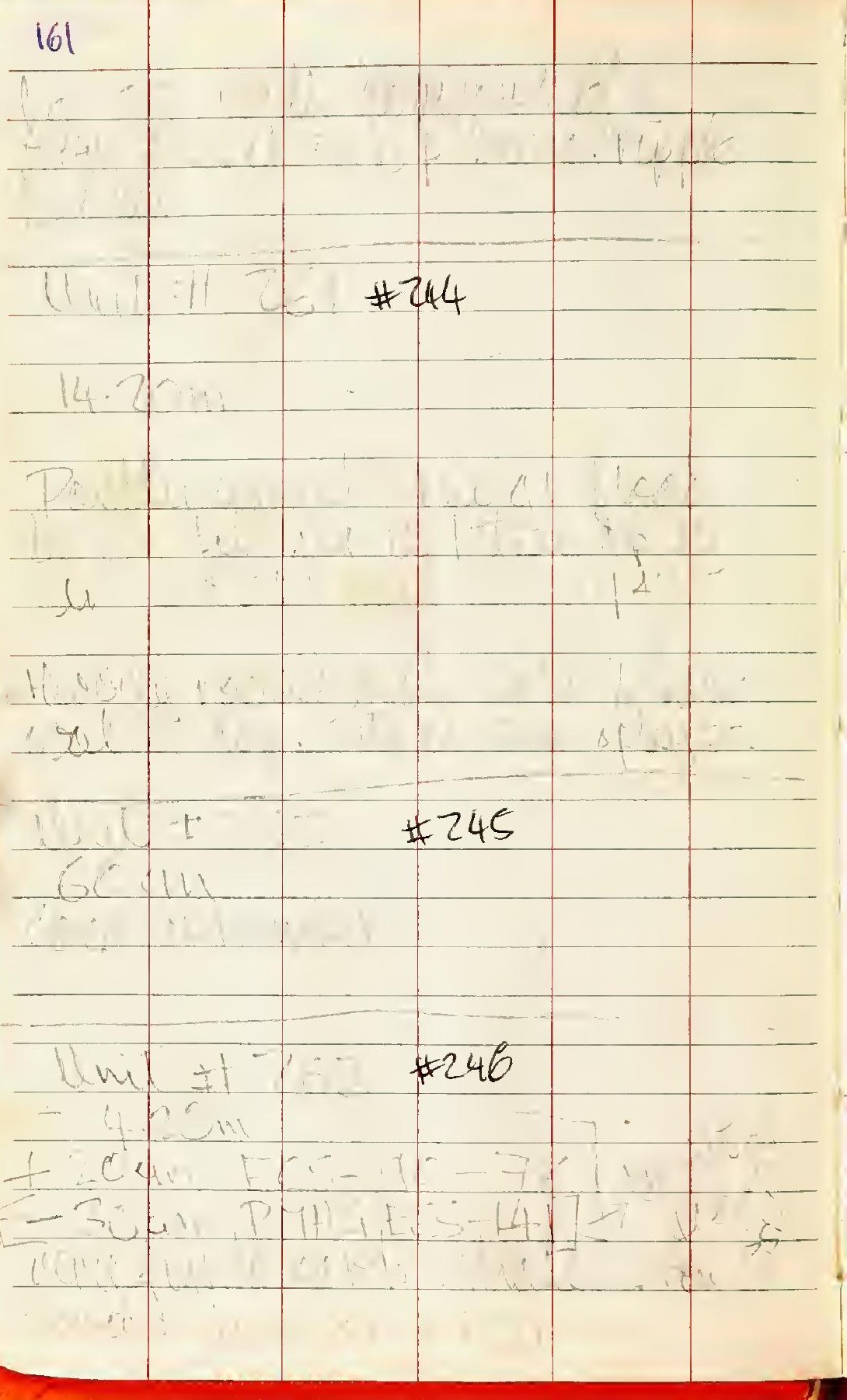


162 \#247

$$
\$ 246
$$

$\$ 249$ 


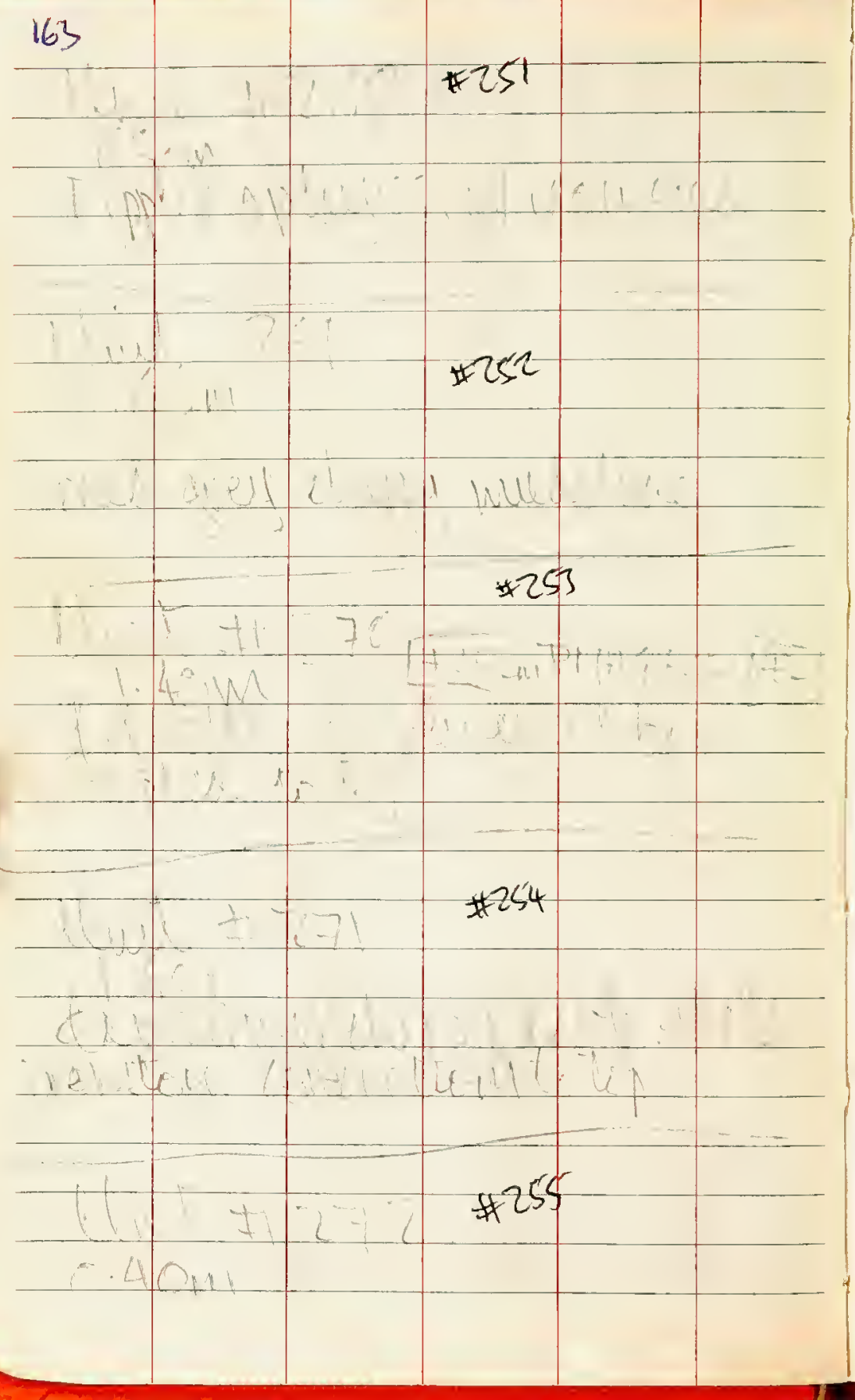


164 


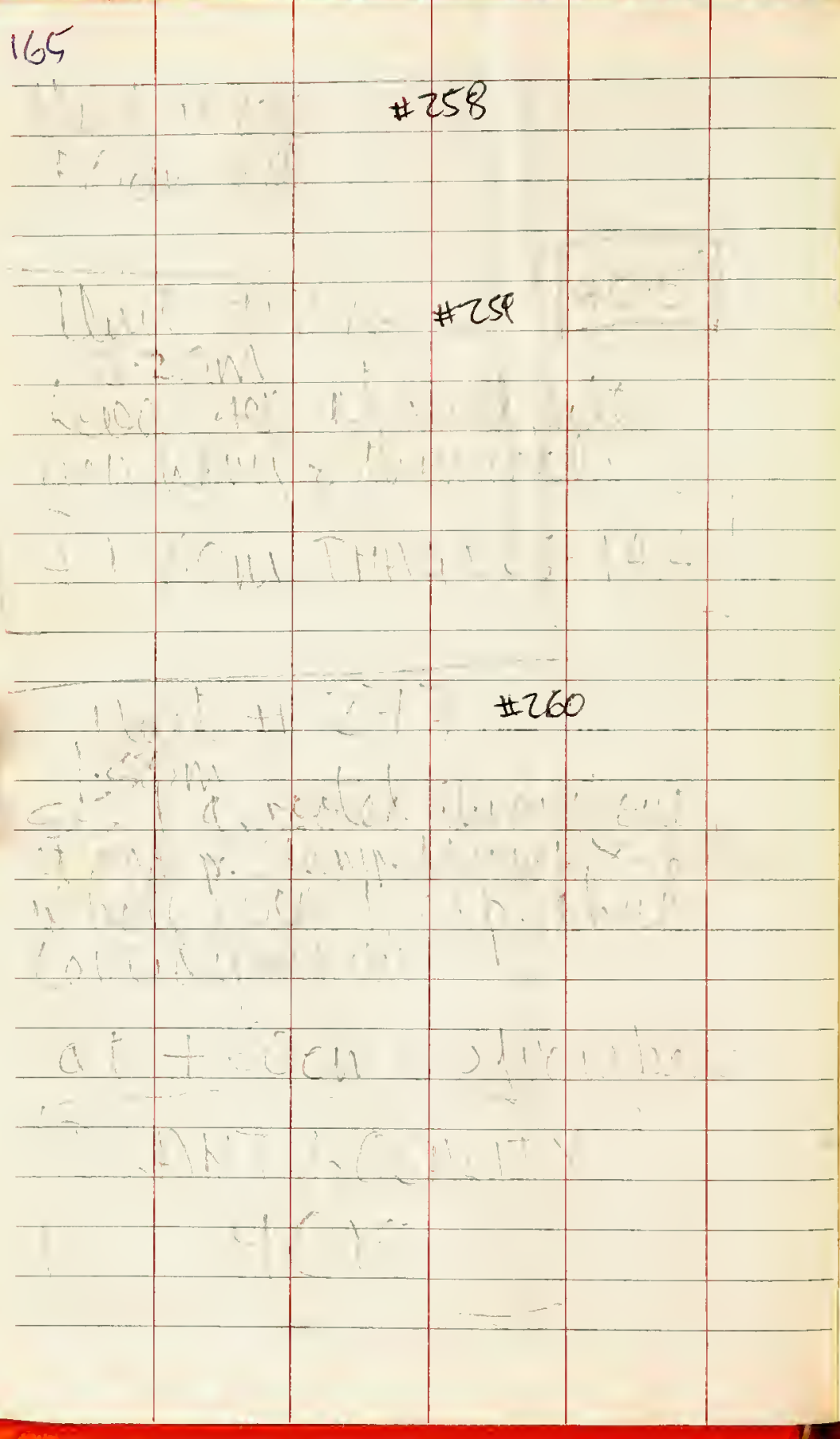




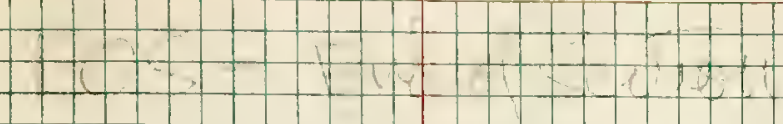

$\begin{array}{r}5 \\ \hline\end{array}$

$-$

$+\cdots+$

$++1$ 





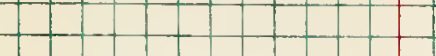

\section{$+-1$.}

$+1$

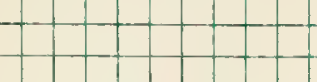

$+1$ 



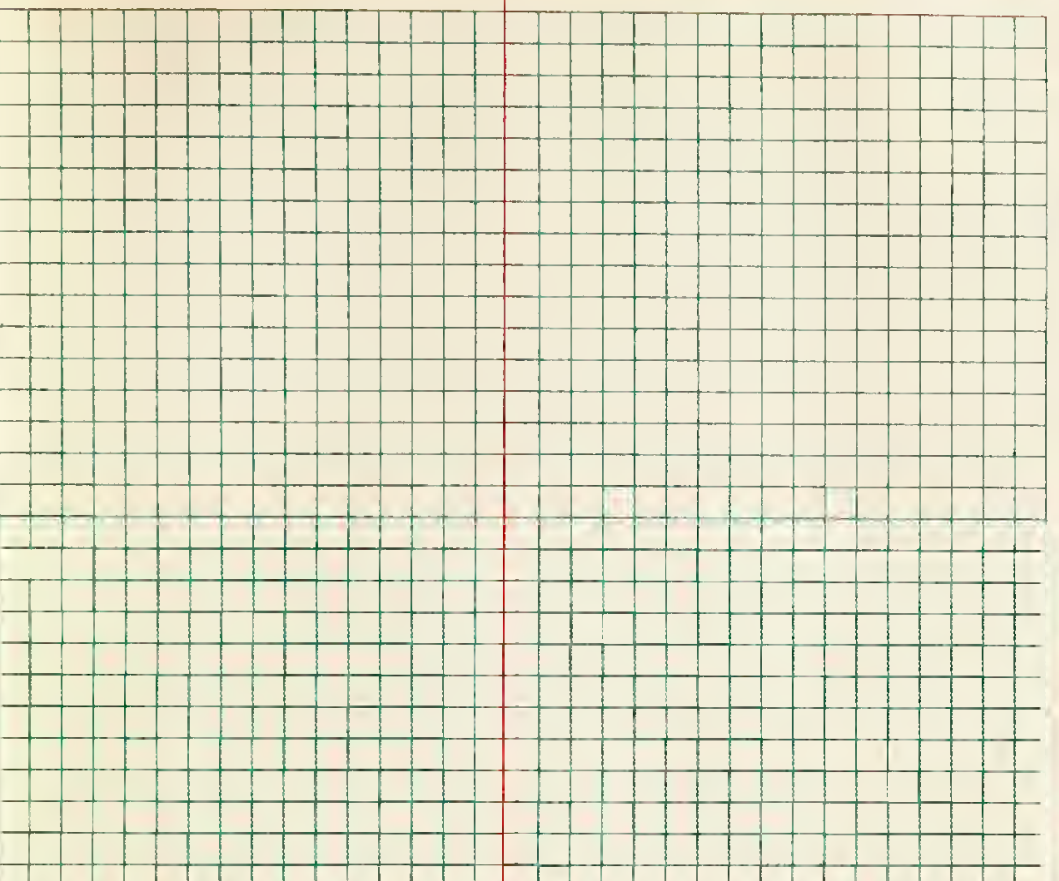

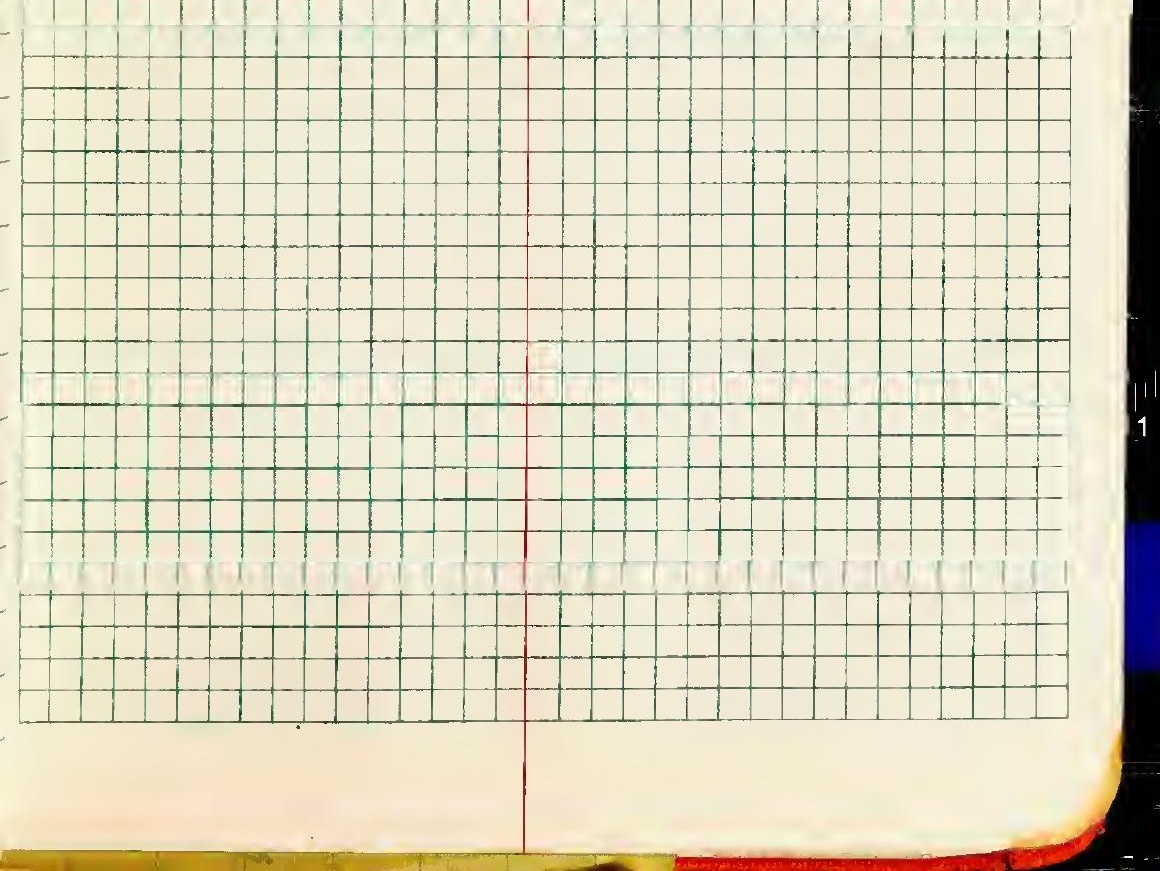




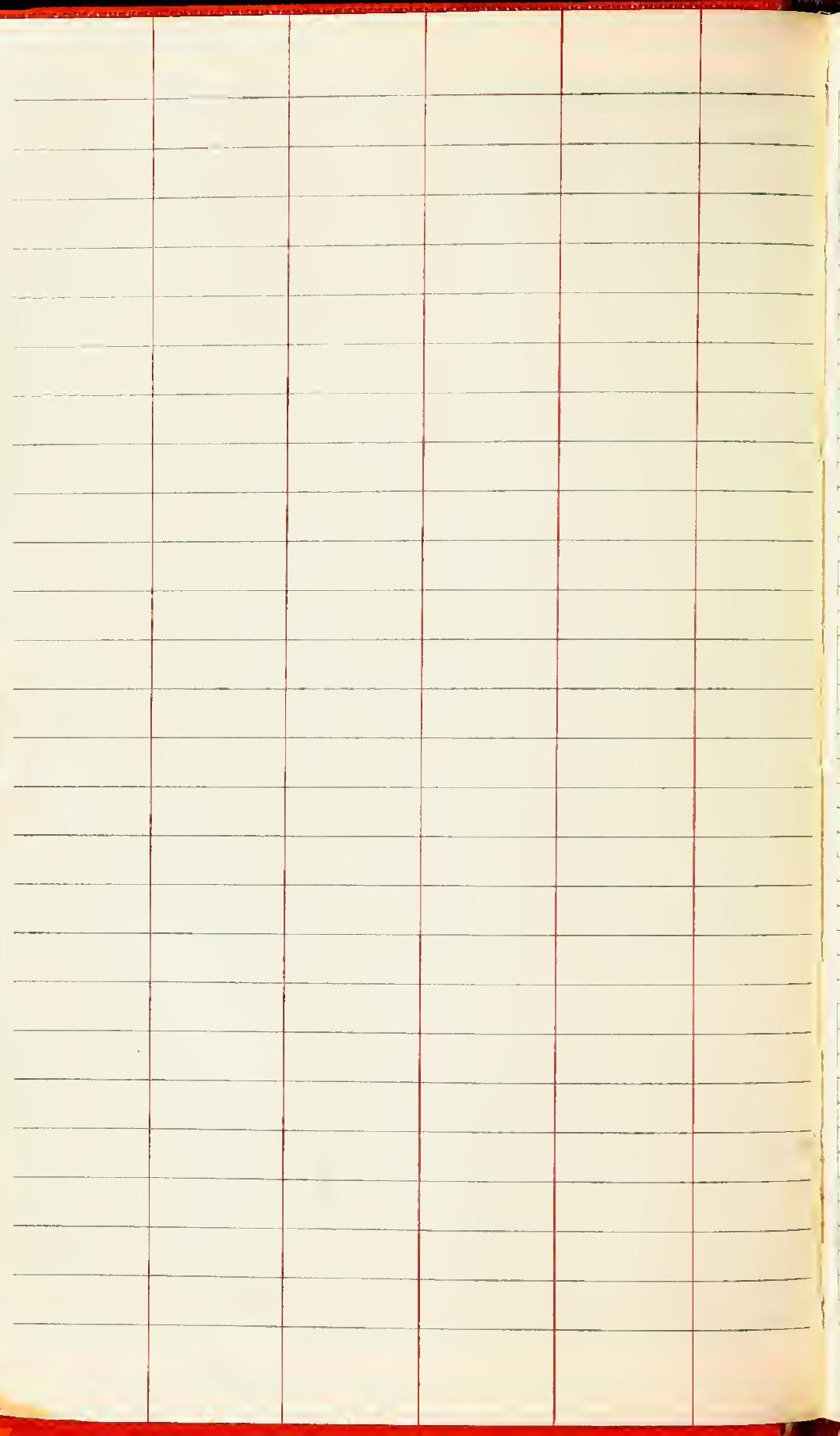





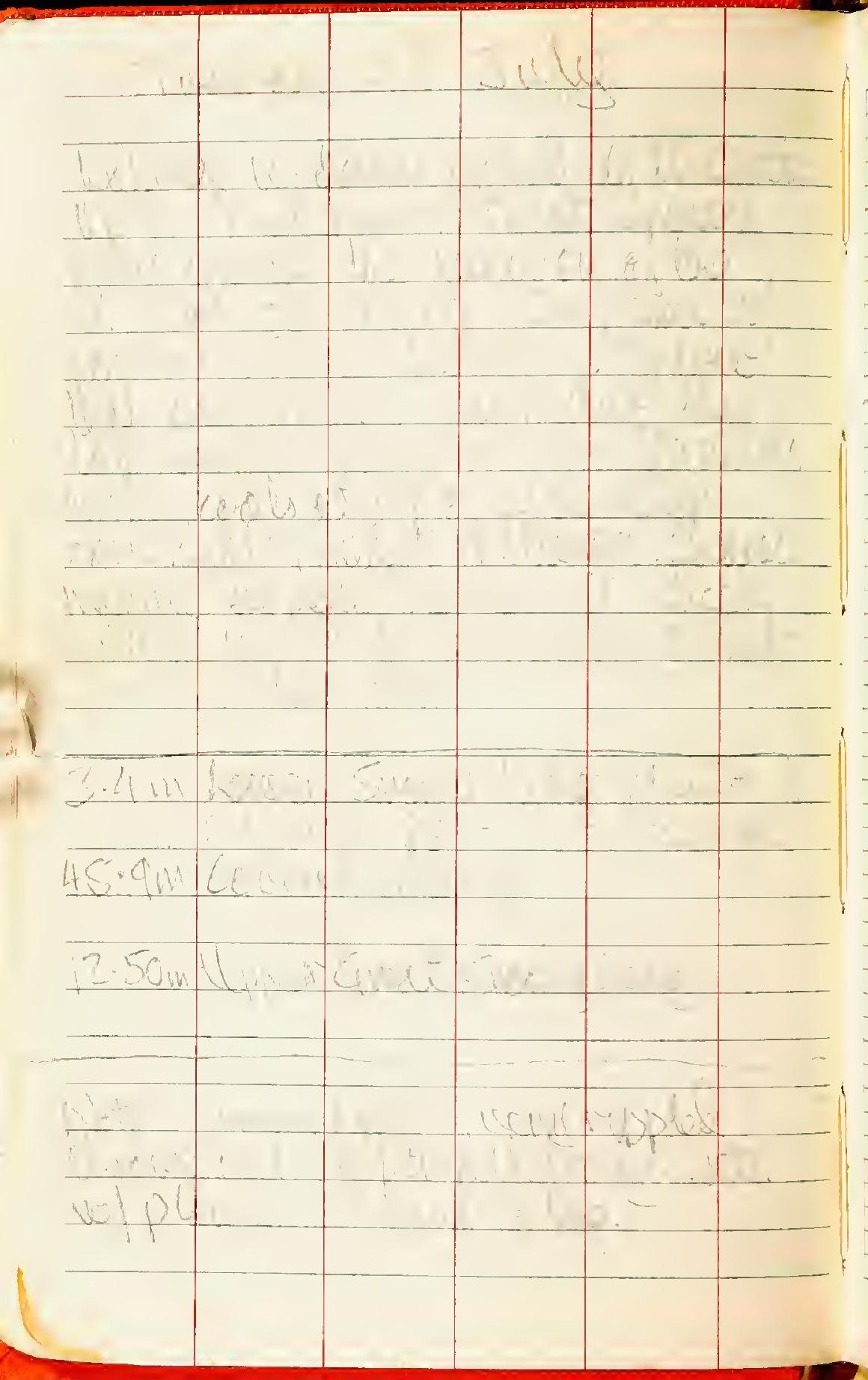




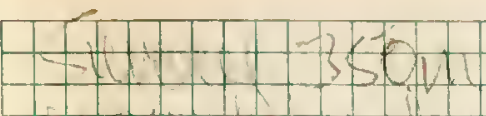

- +1

$D(1)+i+1)$

wat 64

1129.5

$\therefore \quad 48=m=\ln$

$7 \quad 454$ thit

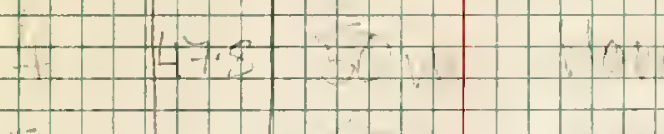

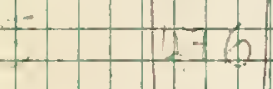

b $+x-1-x+1$

$74 \cdots x$

401401

(4) $\quad 40.1$

\begin{tabular}{|l|ll}
\hline 1 & fectititi
\end{tabular} $380 \cdot 112,7$ 





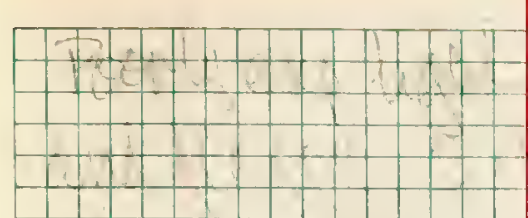
\begin{tabular}{l|l|l|l|l|l|l|l|l|l|} 
& & & & & & & & & \\
\end{tabular}

\begin{tabular}{ll|l|l|l|}
\hline 1 & & & & \\
\hline
\end{tabular}

$+$

(1) +

$+1$ $-1+1-1$.

$+\frac{1}{+1+1}+\frac{1}{+1}+$

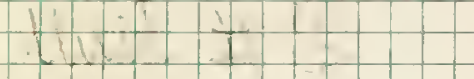

$+1+1$

\begin{tabular}{llll}
1 & 1 & \\
\hline & + & \\
\hline
\end{tabular}

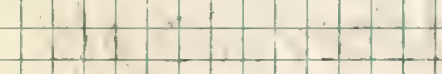

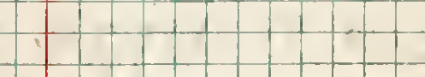

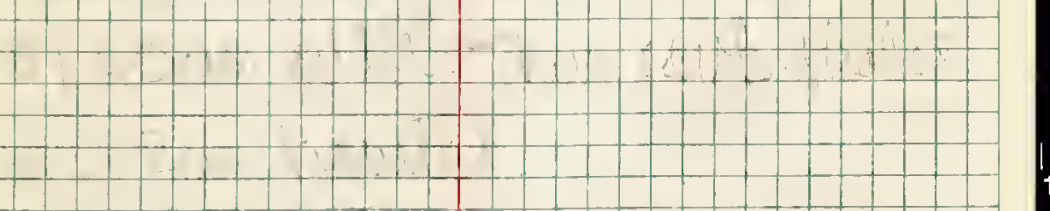




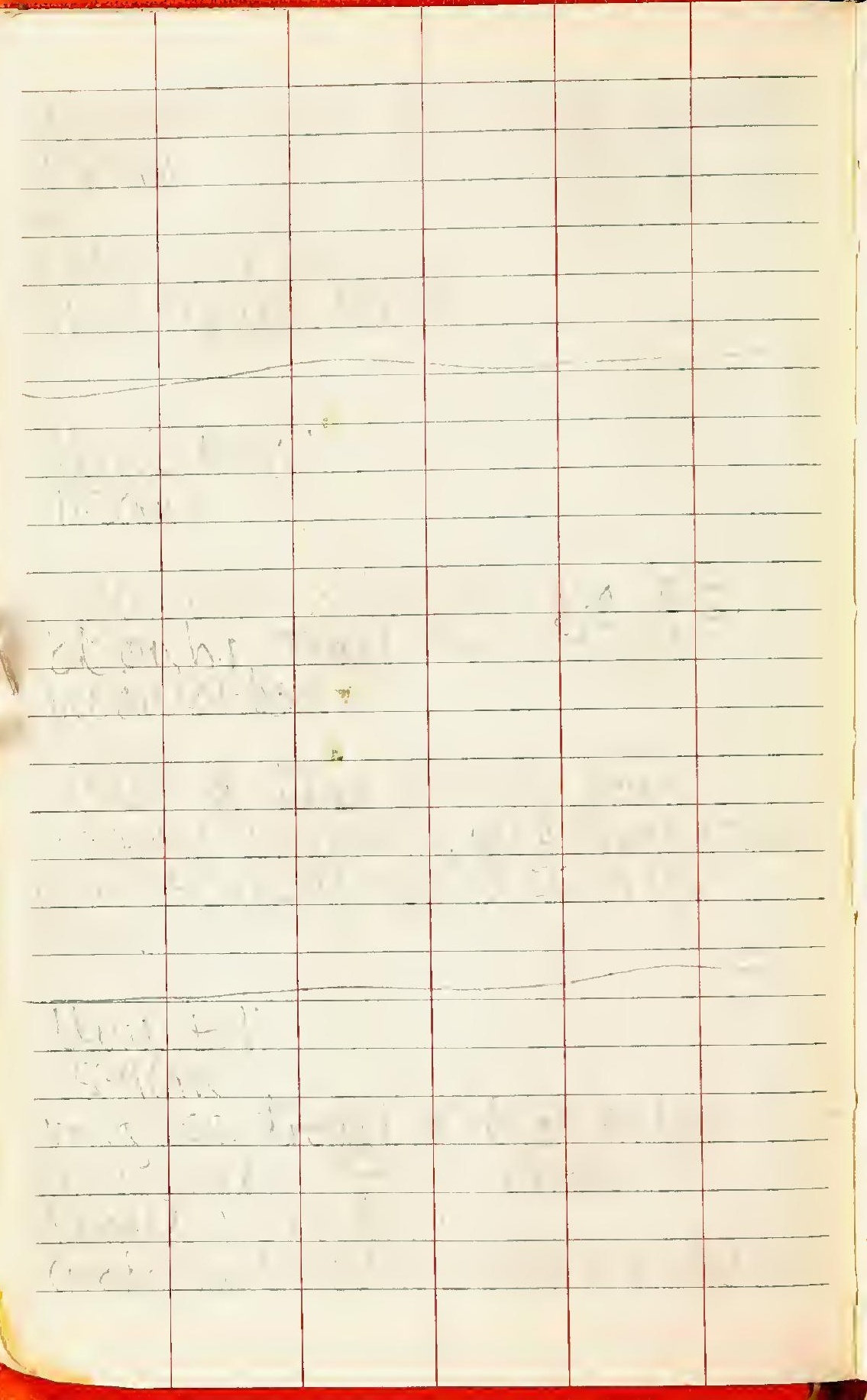






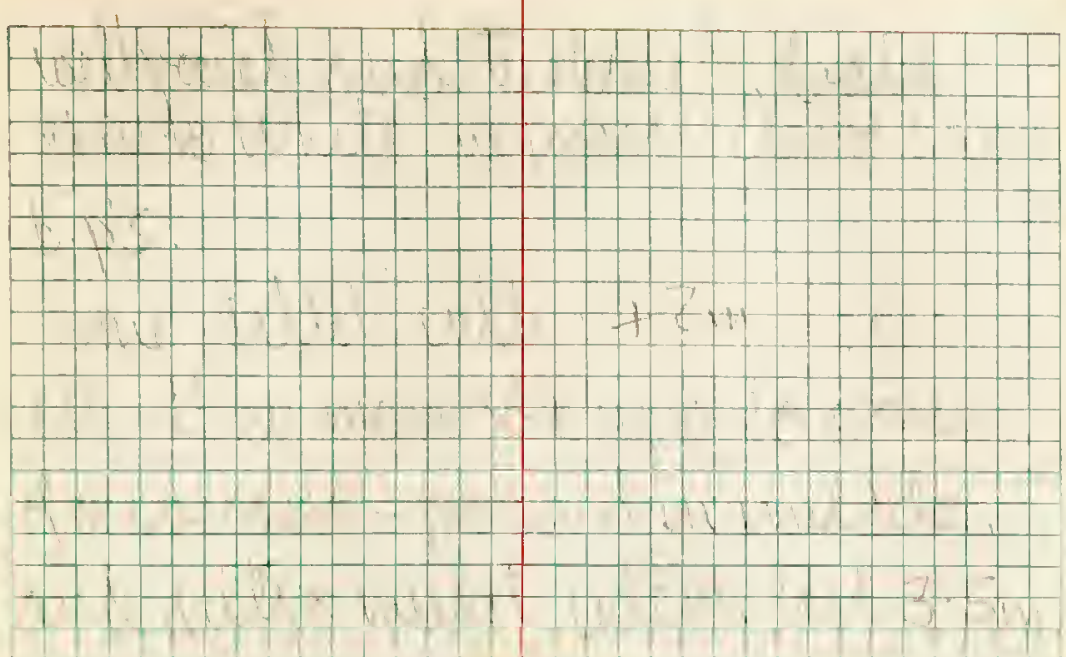

$+1+7+76$

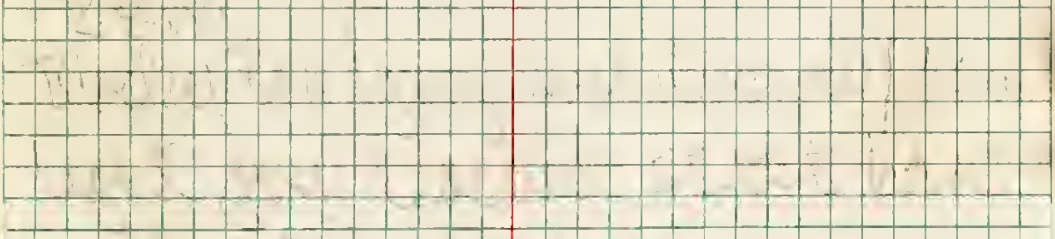

\begin{tabular}{l|l|l|l|l|l|l|l|l|l|l|l|l|l|l|l}
\hline \\
\hline
\end{tabular}

\begin{tabular}{l}
\hline \\
\hline
\end{tabular}

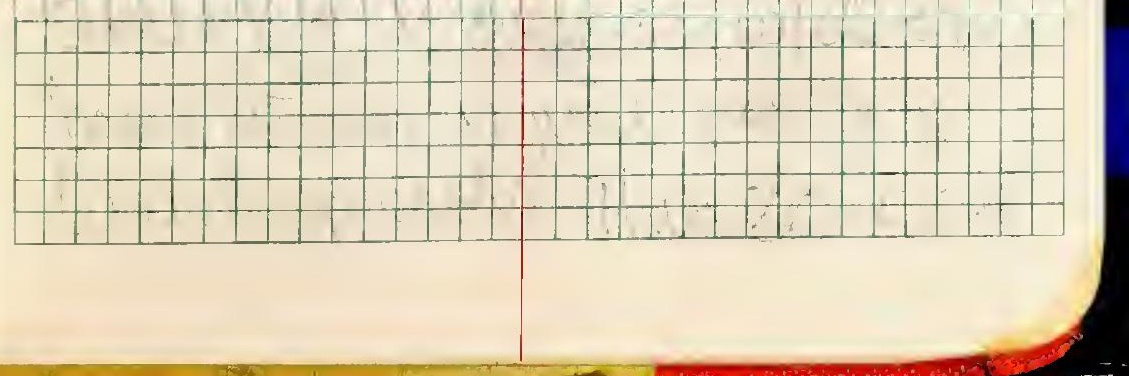




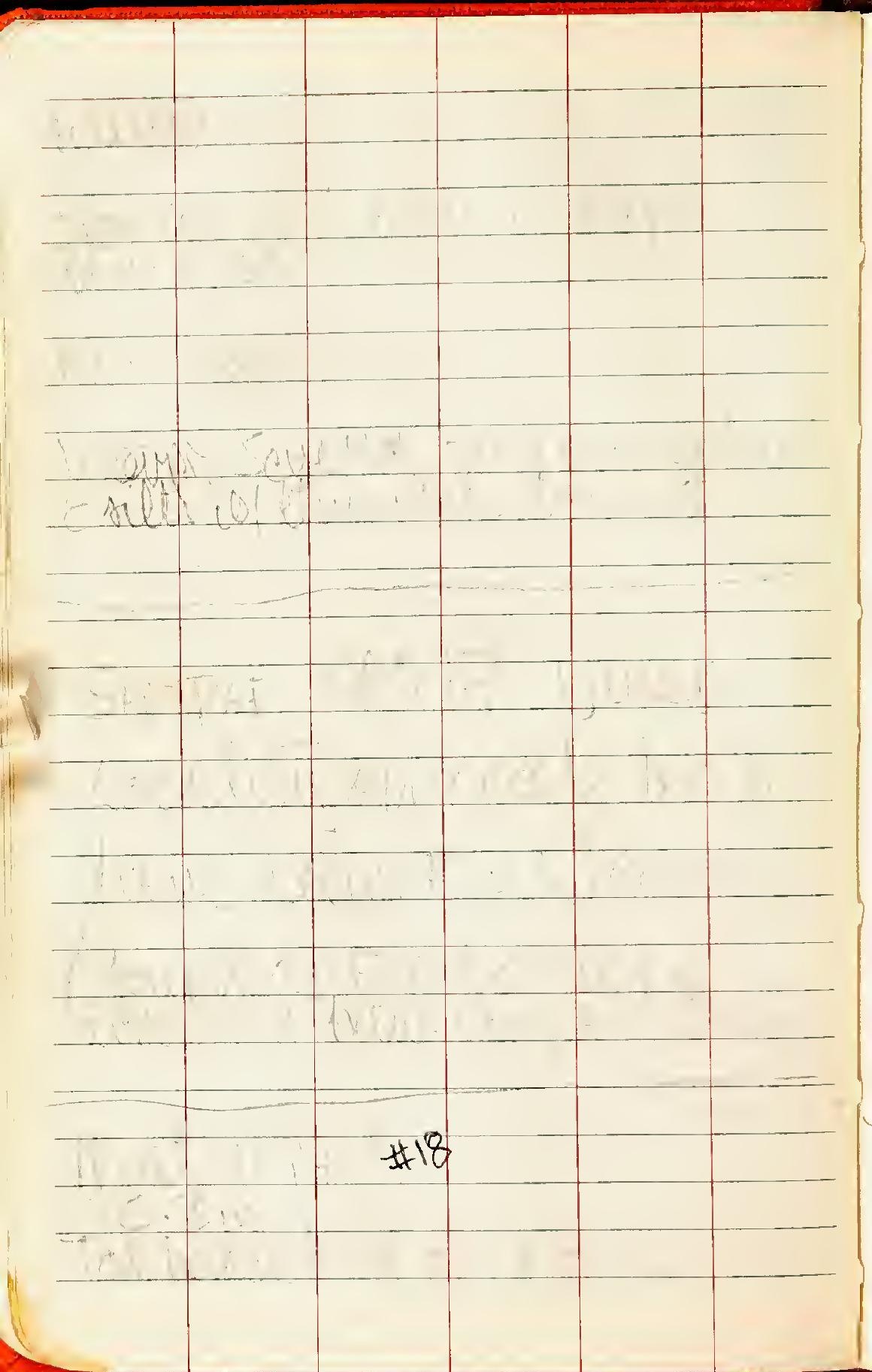




\section{$\frac{1+1}{4+\ldots+13}$ \\ L. 1. \\ in e.

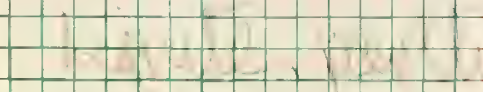

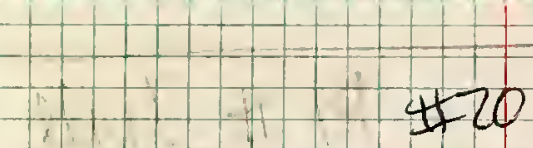

$1+1+1$

$+\frac{1+1}{1}=11$

1. L

$+2$

$+1.12$

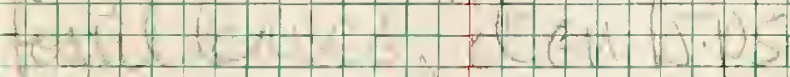

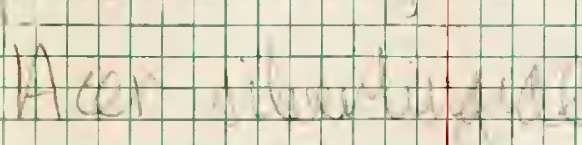

1.1.

$+1+1+f+1$.

$1+1$

$+11.19$

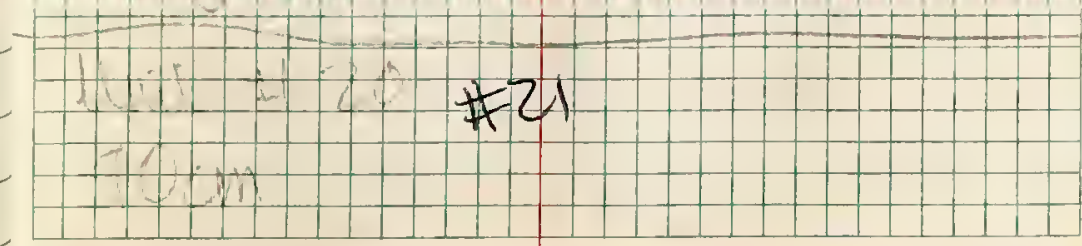




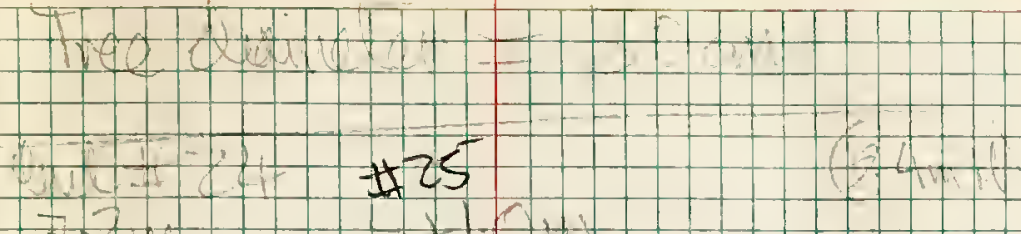
$=7 \cdot 7 \times 1$

$-1+1+0$

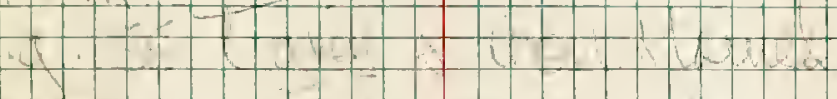

$+1$

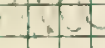

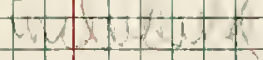

$\{+1:$ it

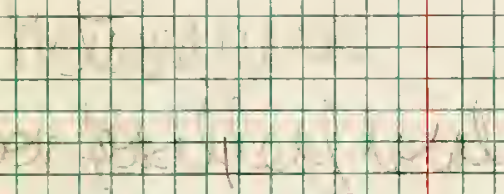

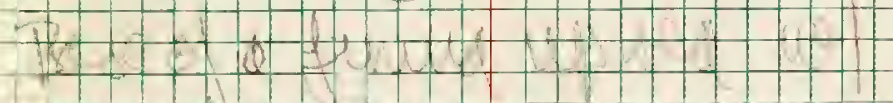

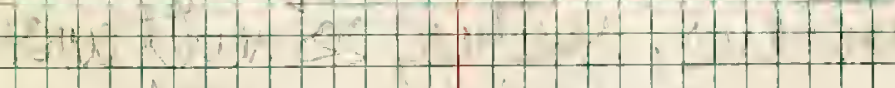

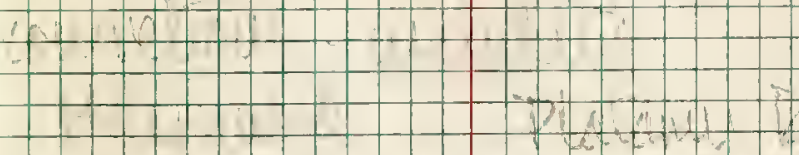

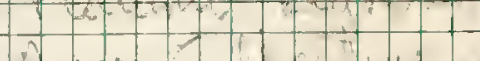

it det?

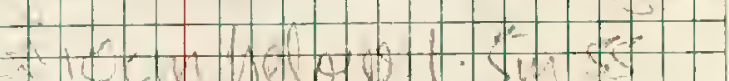

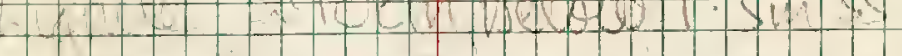

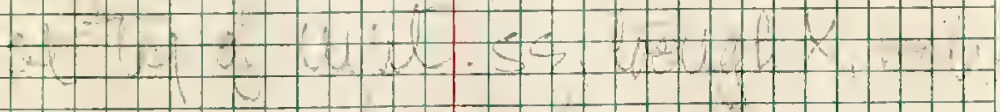

$-4+24$

$-1$ 


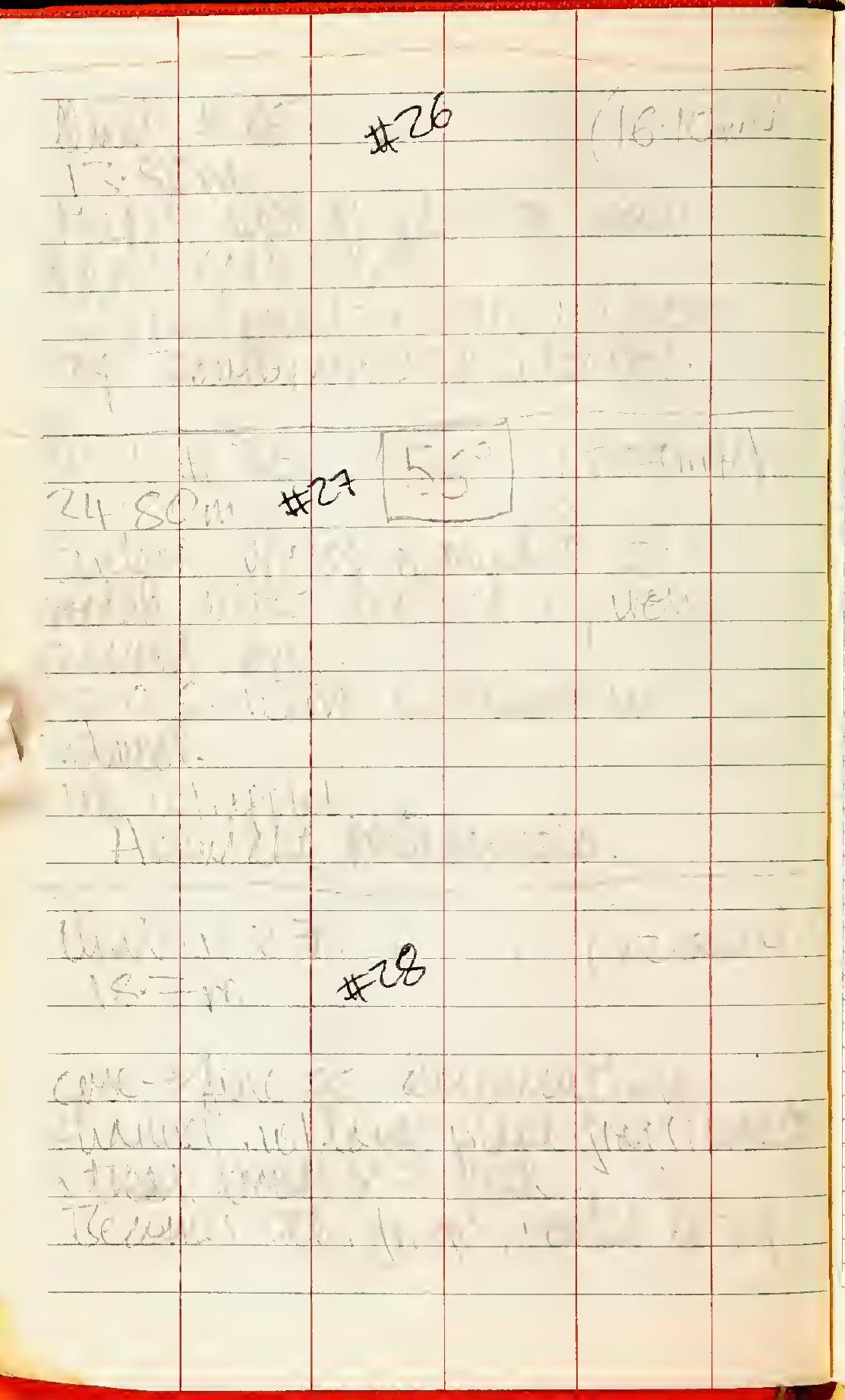






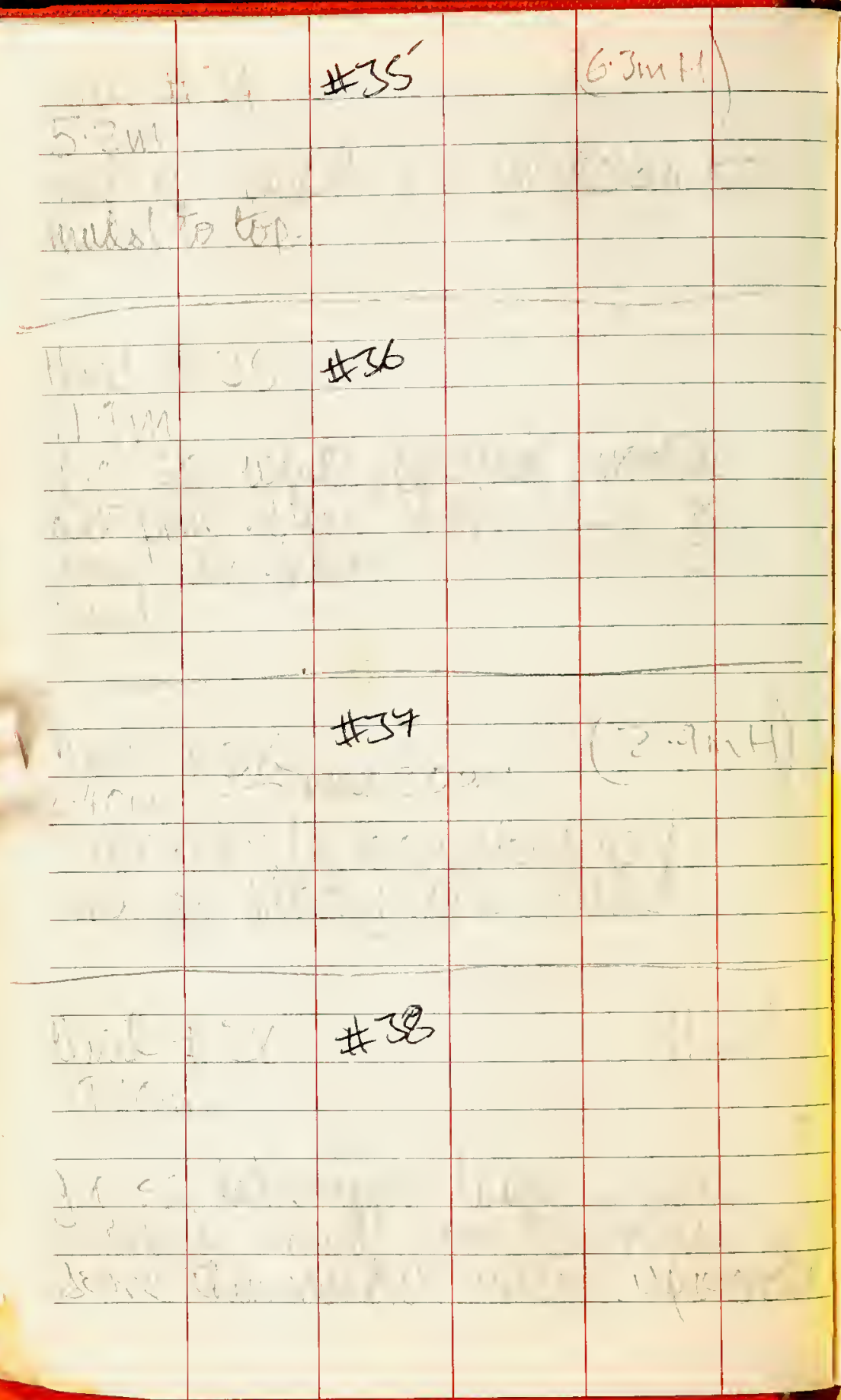




$$
\begin{aligned}
& \text { Unit. } 2 \rightarrow \begin{array}{l}
\text { Unit } \\
28 \text { (Include) } \\
=\frac{244 \mathrm{~m}}{225.55} \mathrm{~m}
\end{array} \\
& \text { Unit } 29 \rightarrow
\end{aligned}
$$





\section{$+1.1+1+2$}

$\frac{1+1}{439}$

$\therefore+1+1$

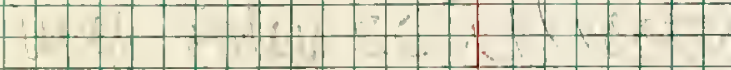

\begin{tabular}{l|l|l|l|l|l|l|l|l|}
\hline \\
\hline
\end{tabular}

Unt + \pm 40

$=1$

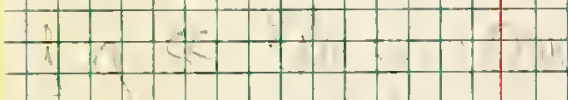

$+1+1 ;$

$+\frac{1}{+1}+1$

$\lim _{-1}$

$+1+1:$

II 


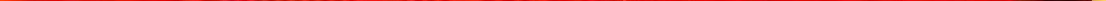


Unimititi-

(ind

setti s-1

$\lim _{1}$

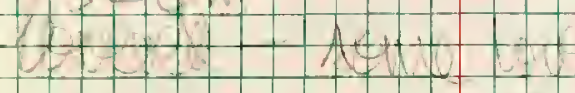

+1.
$f$

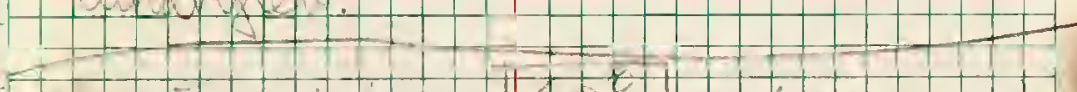

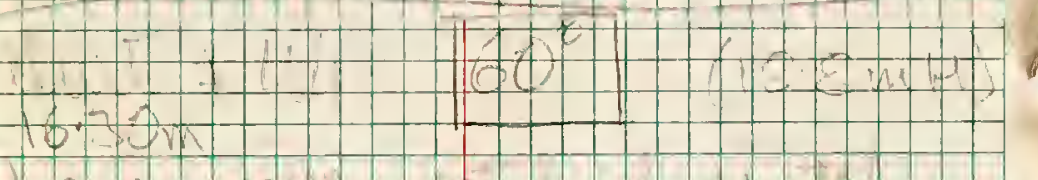

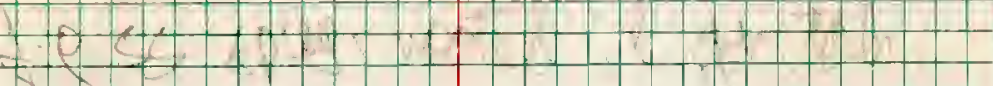
$+1.0101$

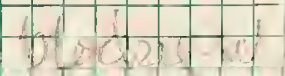

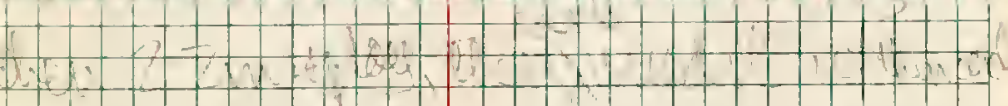

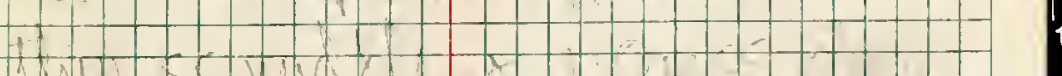



(II) $10+4$

in $\sin$

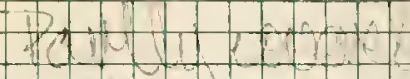

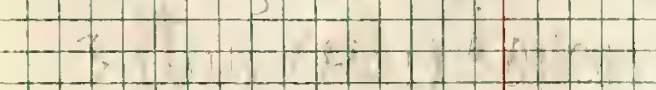

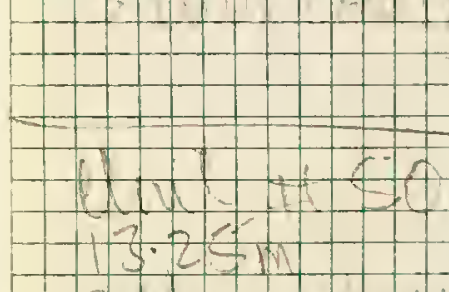

(-1.1. .

- $1: 2+-$

it:

Punji:

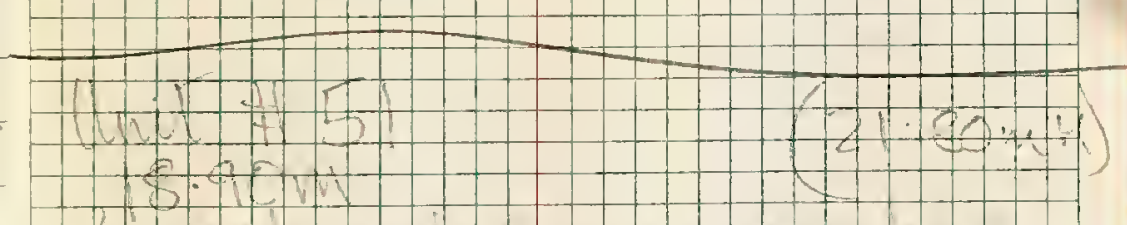

$y_{1}+1 \times-11$

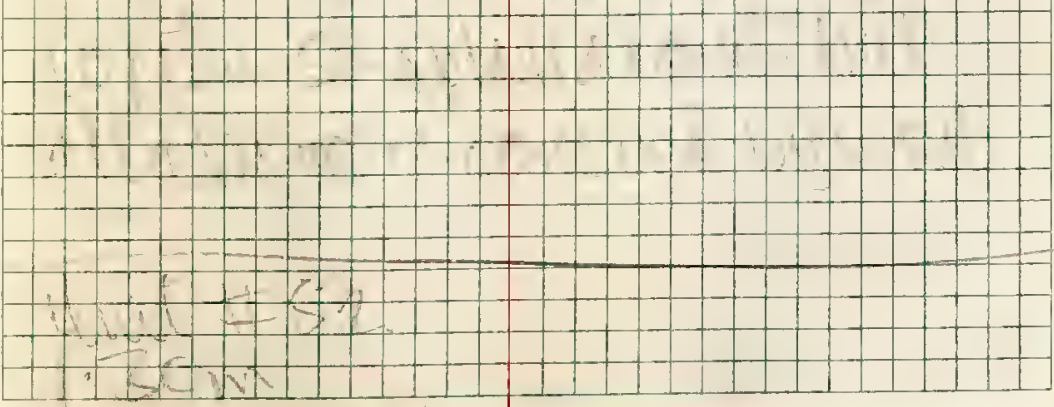



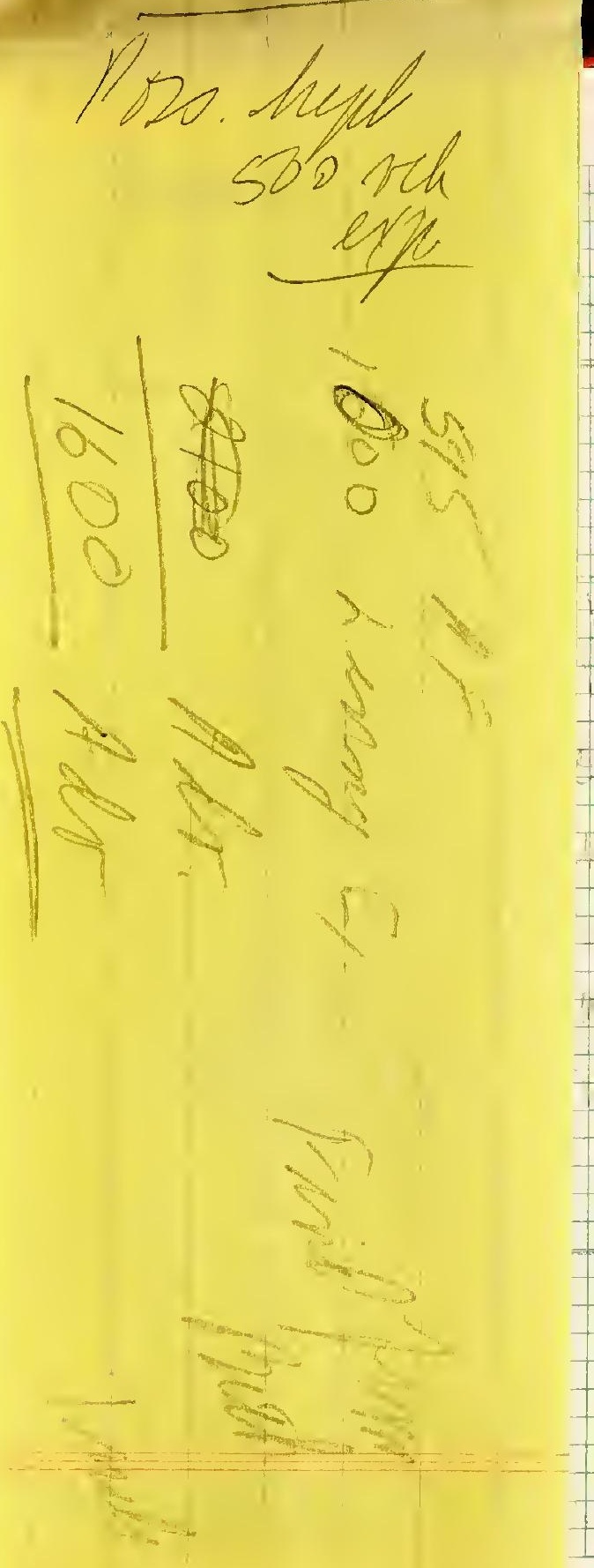


$$
\begin{aligned}
& 2200 \\
& 600 \\
& 700 \\
& 545 \\
& 600 \\
& 700 \\
& 500 \\
& 800
\end{aligned}
$$




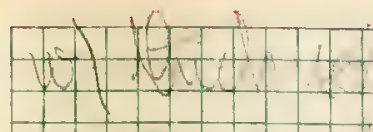

(1) $11+$

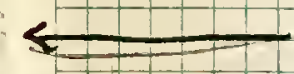

$+\frac{1+2}{b \cdot a c t}=$

$+f(1+\cdots)$

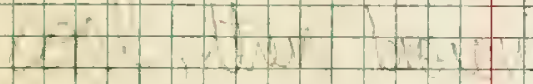

$\rightarrow(0$

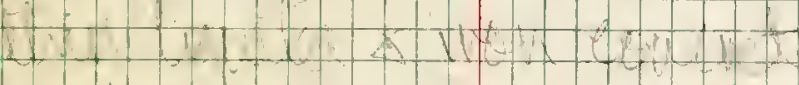

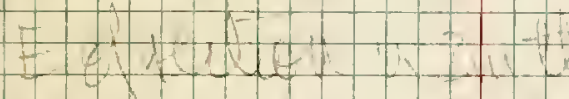

$+1+x+1:+1+1+1$

(4) $(x+1,+1)$

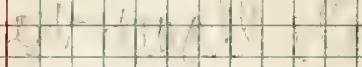

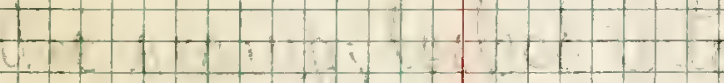

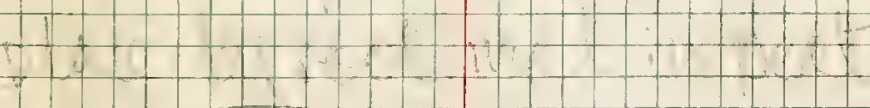

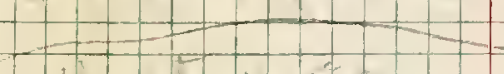

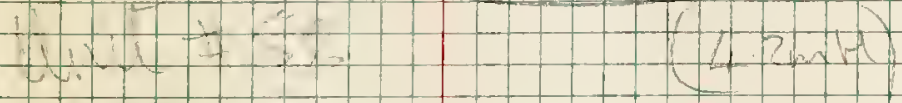

$+2+1+1$

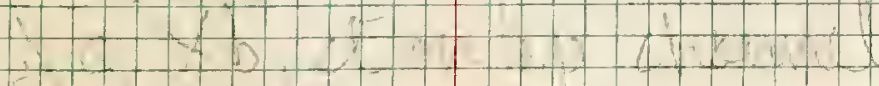

$(A+1)+2+2+3+1+1$ 


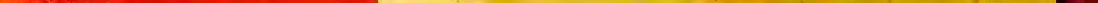




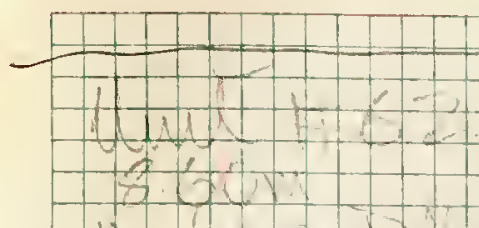

Hent il Mecit

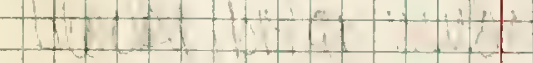

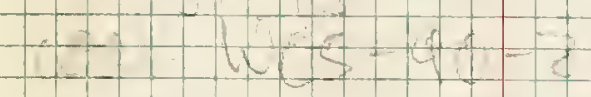

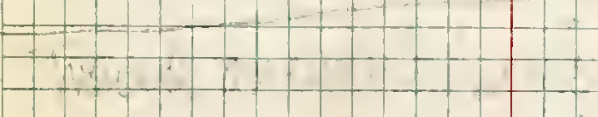

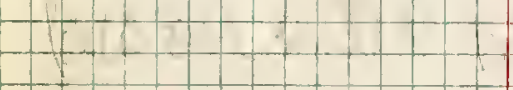

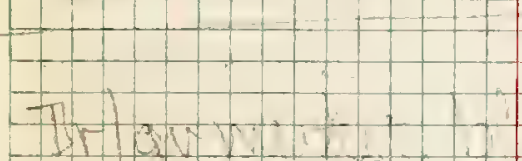

$-11+\operatorname{lot}+2=1$ i.

\section{$+10$}

$+1+\cdots+1 ;$

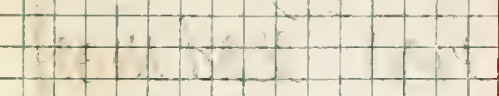

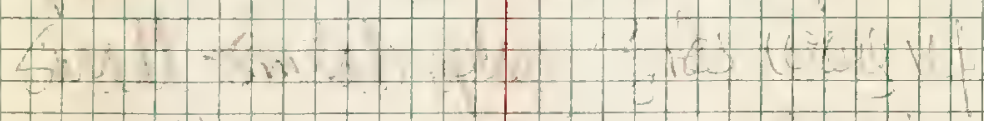

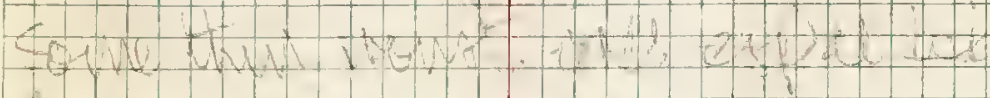

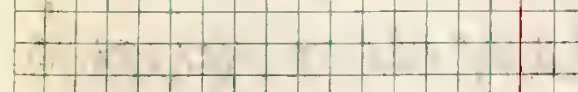

$-1+2+2-1+2$

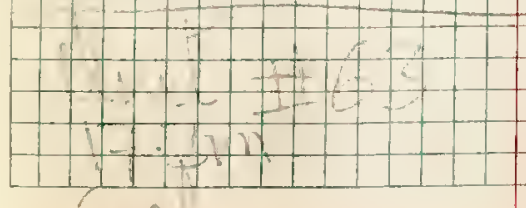
enf $x$ 

Linam

tinet

$-1, y+\ldots$

$-$

(1) D,

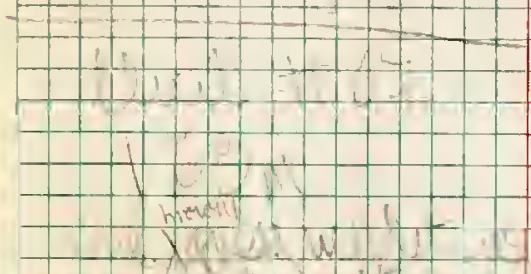

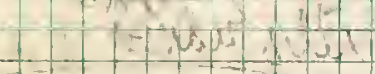

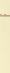

\begin{tabular}{|lll|l|l|l|l|}
\hline & & & & & & \\
\hline
\end{tabular}

1

$+1$

\section{T}

$p=3 x^{2}+1+1+1$

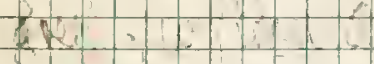
$+1+1$

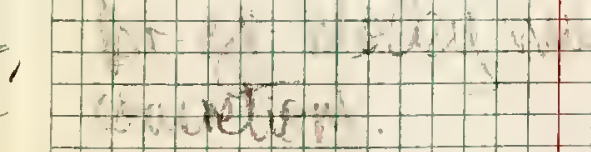

$$
\begin{aligned}
& \left(i+y^{-3}-1-72\right. \\
& \text { L. I I I I I }
\end{aligned}
$$

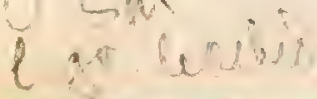




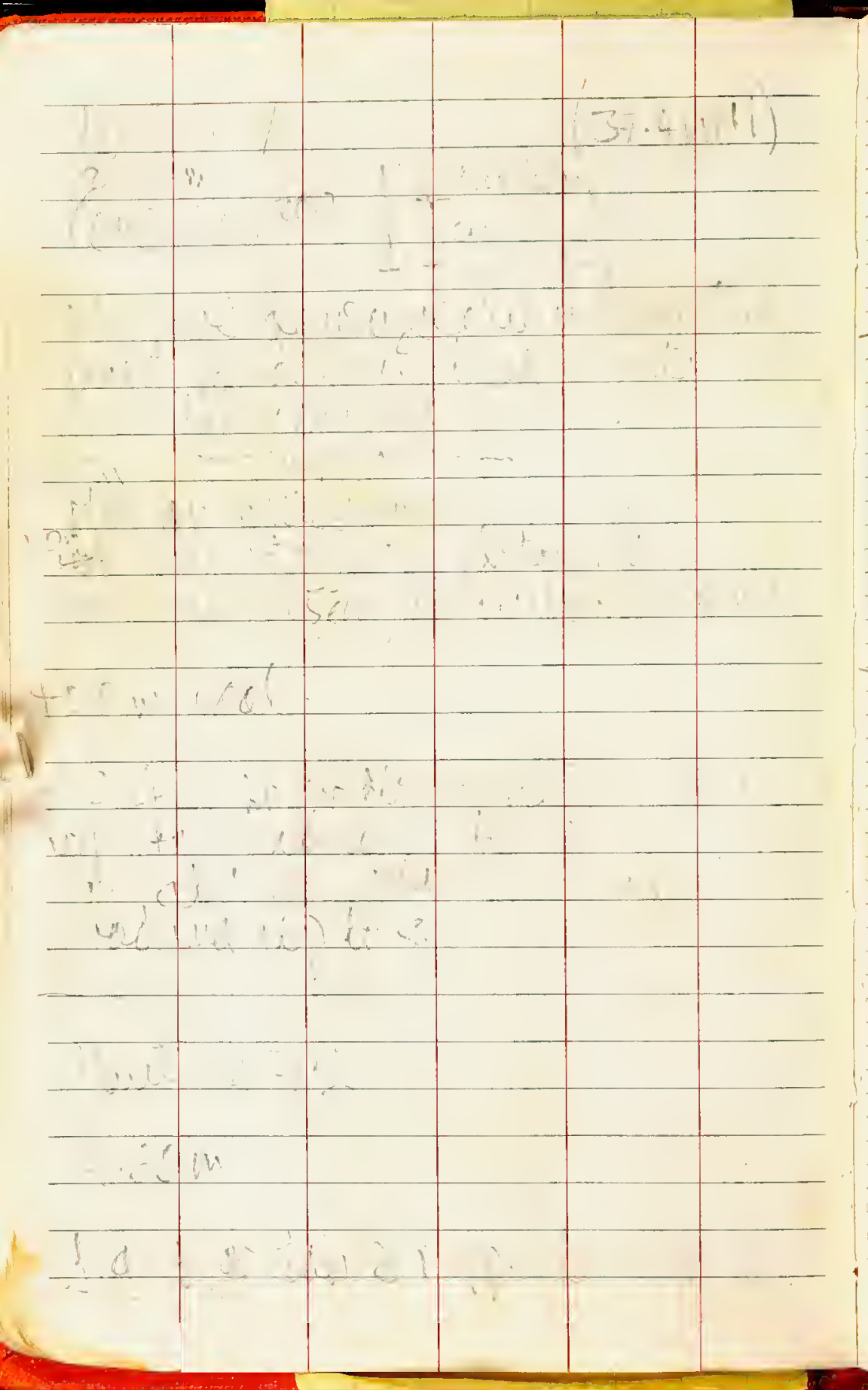


HWTH

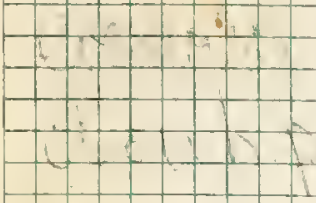

-

.$+1 .+1 .+2$

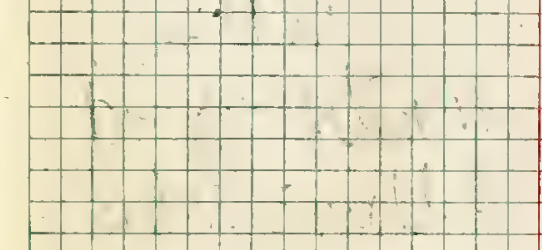

$+1$.

$+$

1

T.

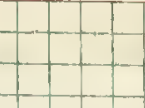

12 ?

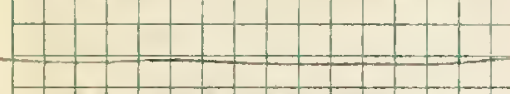

$4 \div+7+4$

$6 m^{2}=1+1$

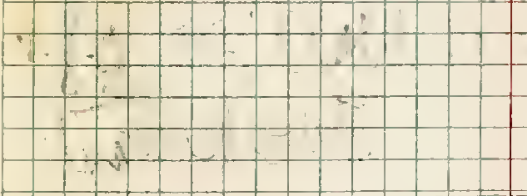

$+$.

3.7.7i:

Ditititis

(

alles. 


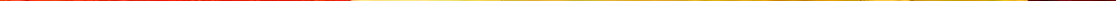



Total triclevess Friduy $=23.9 \mathrm{~m}$ 
Fossils from 9019

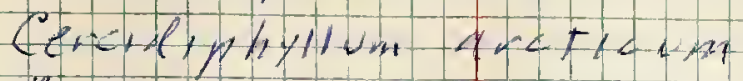

Acer

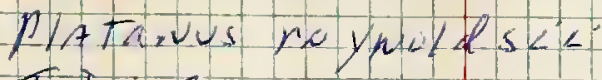

Tilia?

Myrata aff hrestafuritchij

noo somalia

seweral whens

Artula? 

III

$+4$

$+$

$\left\{\begin{array}{l|l|l|l|l|l|l|l|} & & & & & & & \\ \hline\end{array}\right.$

:-

\begin{tabular}{l|l|l|l|l}
1 & & $?$ & \\
\hline & & &
\end{tabular}

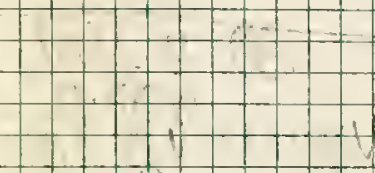

$+1+1+1)$

- Nive

$\frac{1+\ldots+1}{1+1}=$

1.

$+7-1+$

I +4 a

1. $+1 .+40$

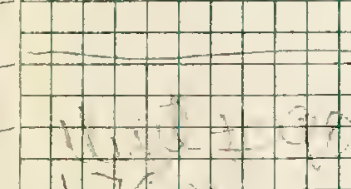

1. 7511

-

$7+\ldots$

$-2+x+1+1$ 


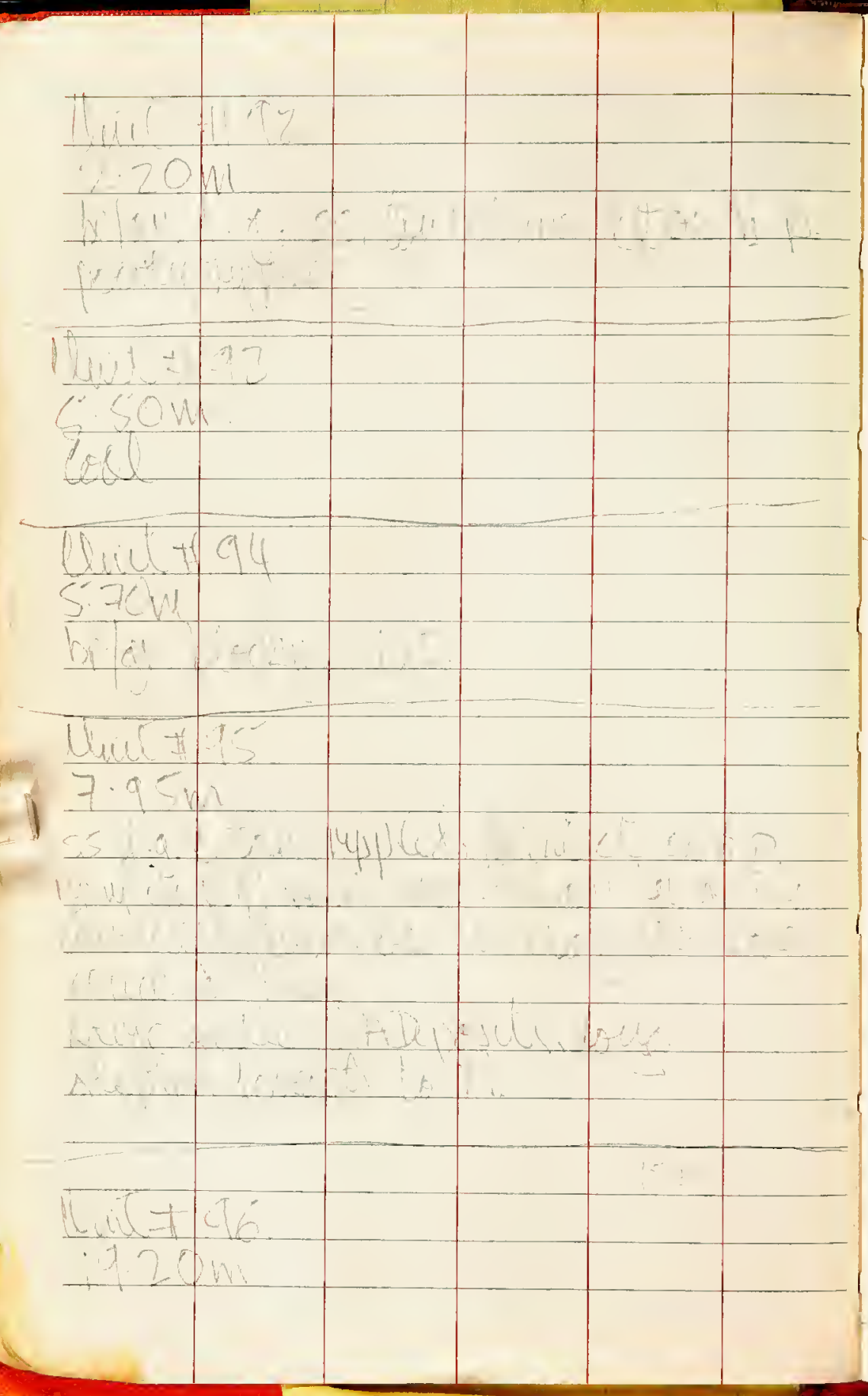






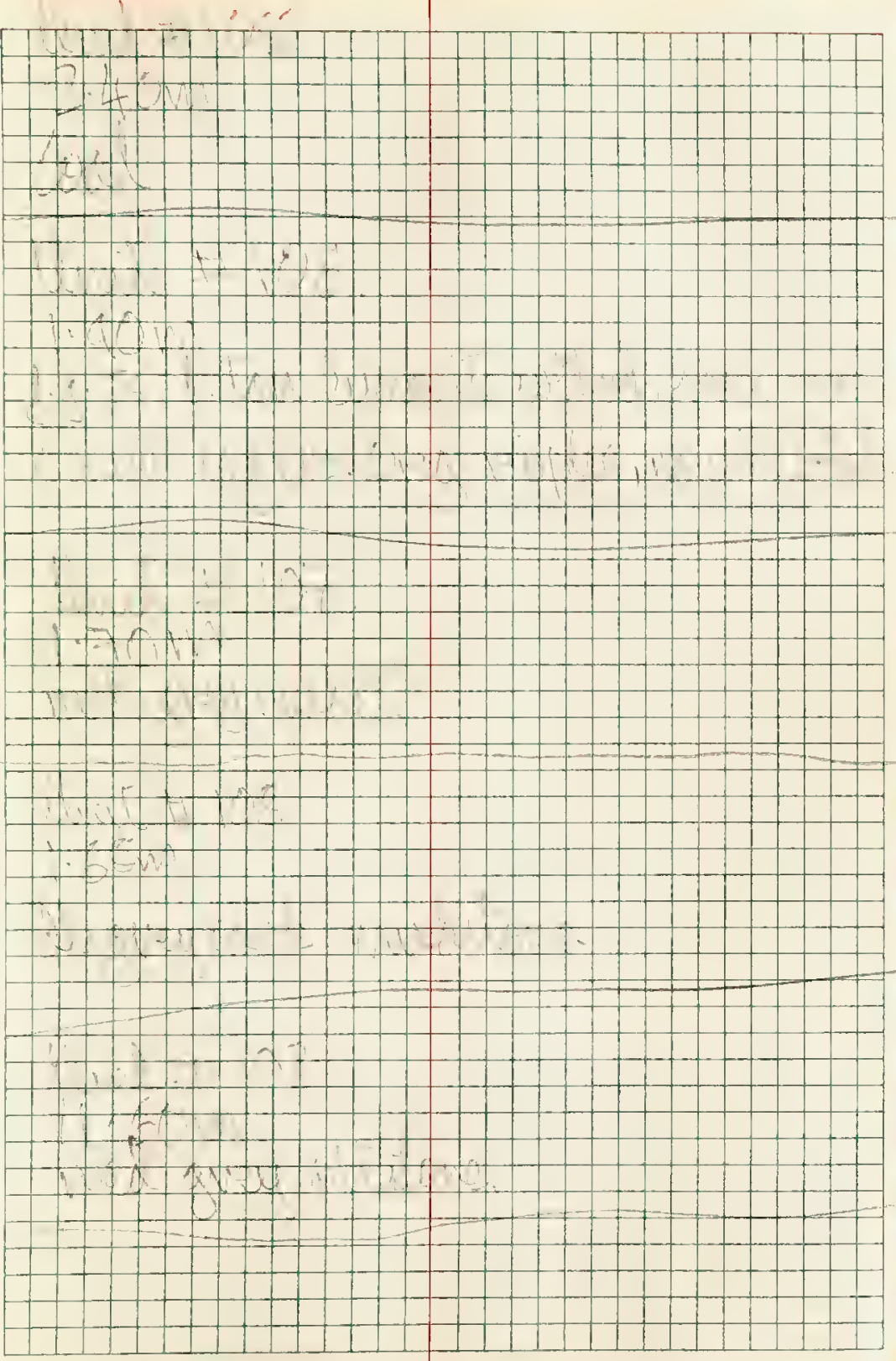




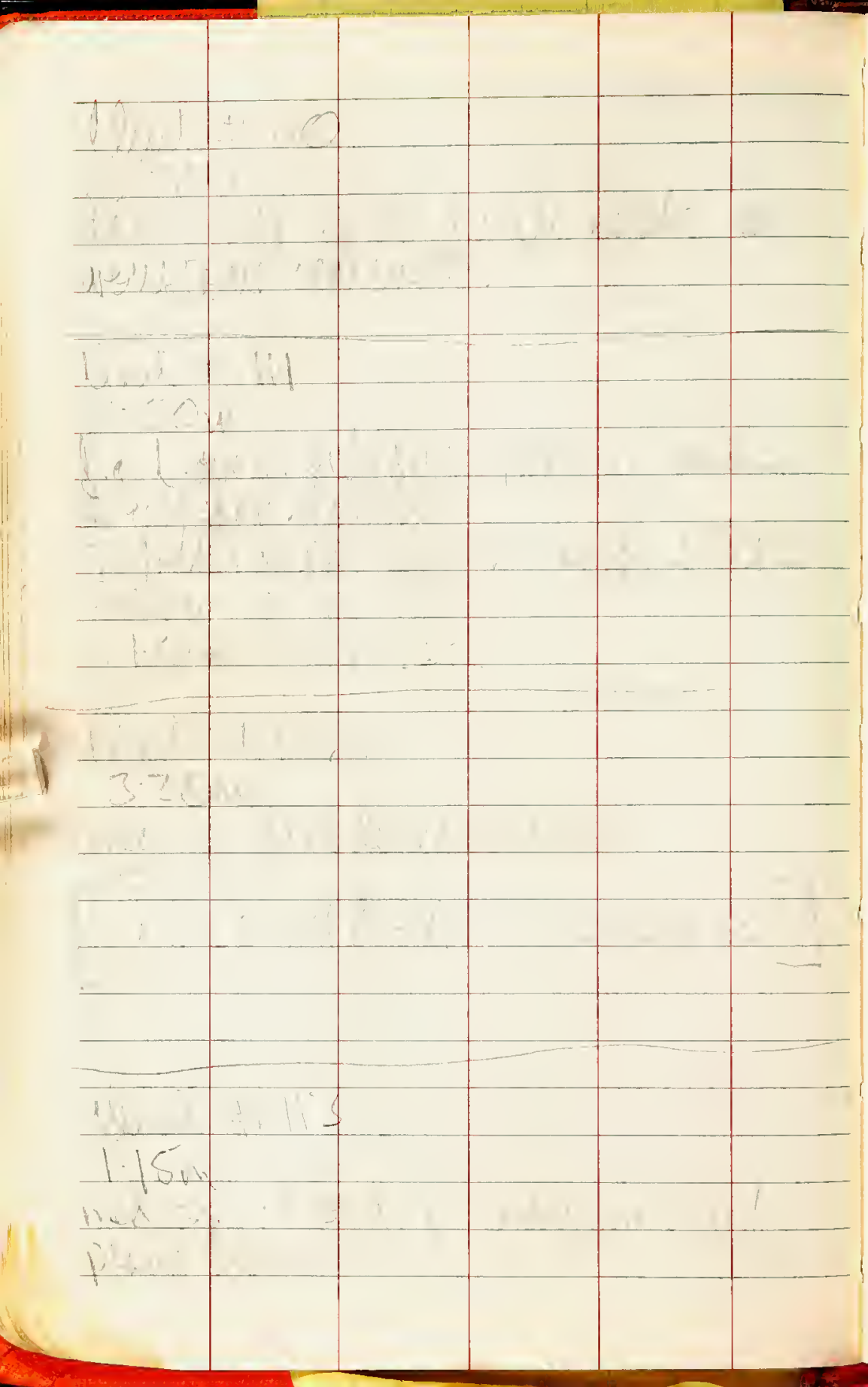


\begin{tabular}{|l|l|l|l|l|l|l|l|l|l|l|l|l|l|l|l|}
\hline & & & \\
\hline
\end{tabular} 


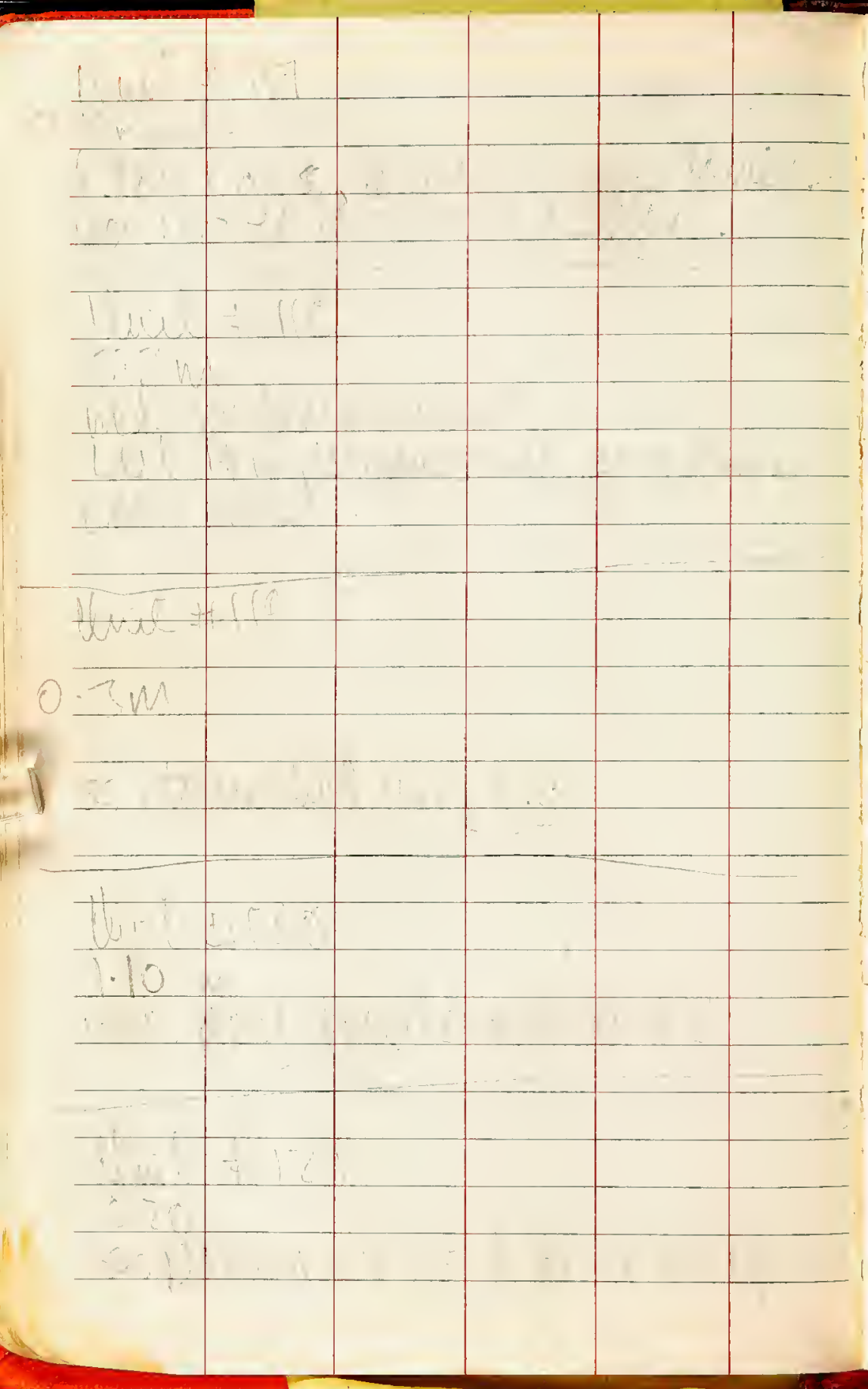


D. -12

$\sqrt{1}$

(1)

$+5+2+1+2$

$\frac{1}{1}$

$+1.1+1$

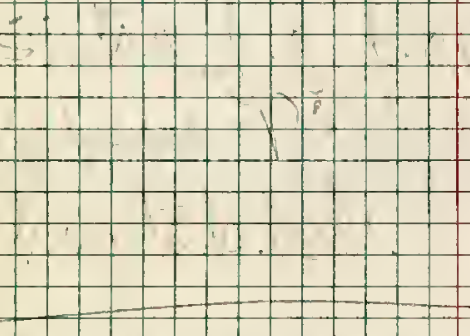

-......

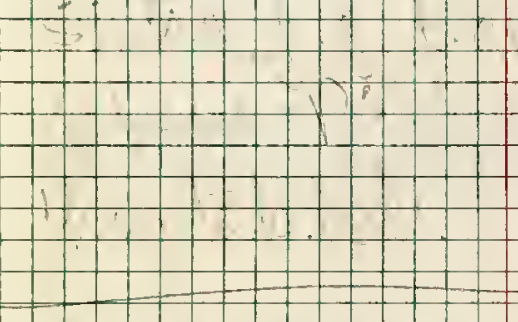

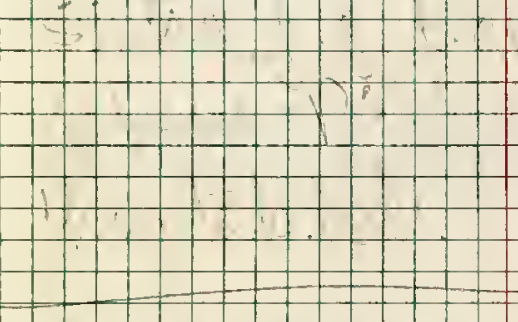

1

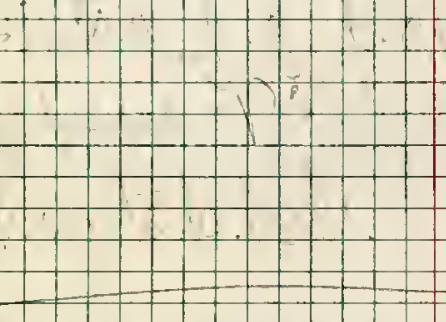

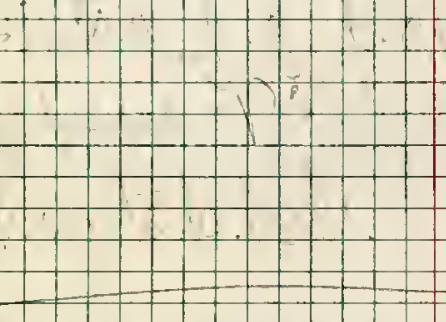

$412+13$

\begin{tabular}{|l|l|l|l|l|l|l|l}
\hline & 1 & & & & & & \\
\hline
\end{tabular}

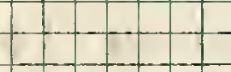

$1-1 ! i$

$+1$

1

1

.

$-1+$

$\left.-1 \frac{1}{1}\right\}$

$+19+\pi$

$1+5+10$. 
Total for sateridal $=152.8 \mathrm{~m}$

TOTAL WEST GREEK SECTION =757H 


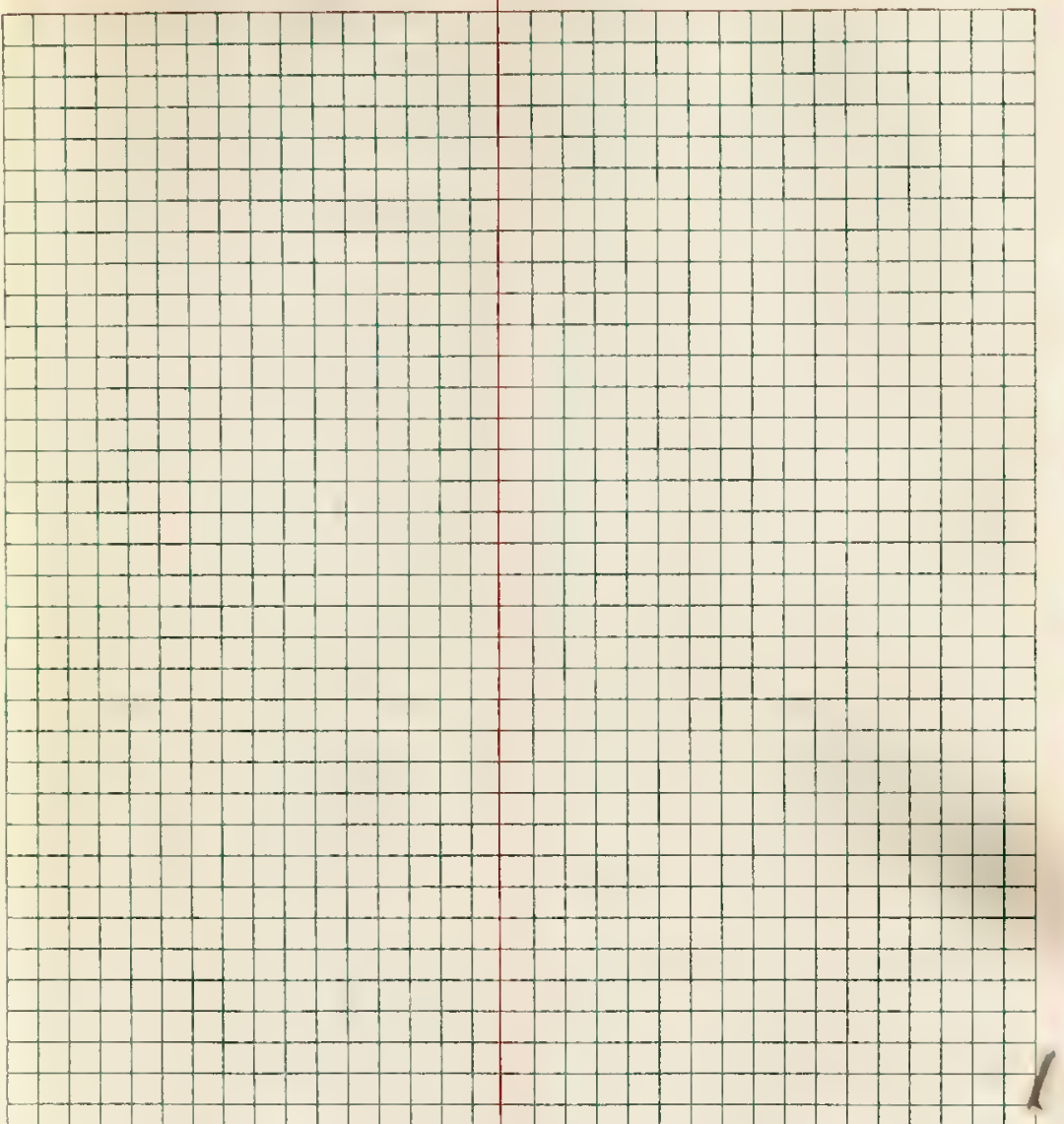


Mendeng 7129190.

up Easl Greete to horate

SITES-

ECS -24
Fect

ECs -26

ECS -42

$+25-60$

F⿻ 132 

Note: ever in locality number, q0zohas boanued ona Platan nobius piere of flat. Thes Horell nou be ve-uembened 9021. 9020 voill ventin the benterale taxilie abee the Strand Fionel Fenvidetion.

$$
\begin{aligned}
& 9022 \text { las hoen ured fer } \\
& \text { Torvefloppice, llut \#146,p.102 }
\end{aligned}
$$


SITE VISIT to mes?modps

Dexeloparat sife; sw. df intensec.

of TodA SA, Whined Ane

North antanct

vitTh MEN MN

mandine?

Whlkell to bidy twe stope

3!! Soff Antoric is, Congl.

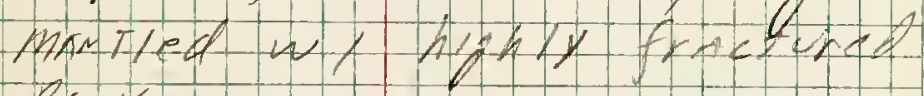

Roek.

- PrNAY

Collected G SAMples

LVA 9022 . 


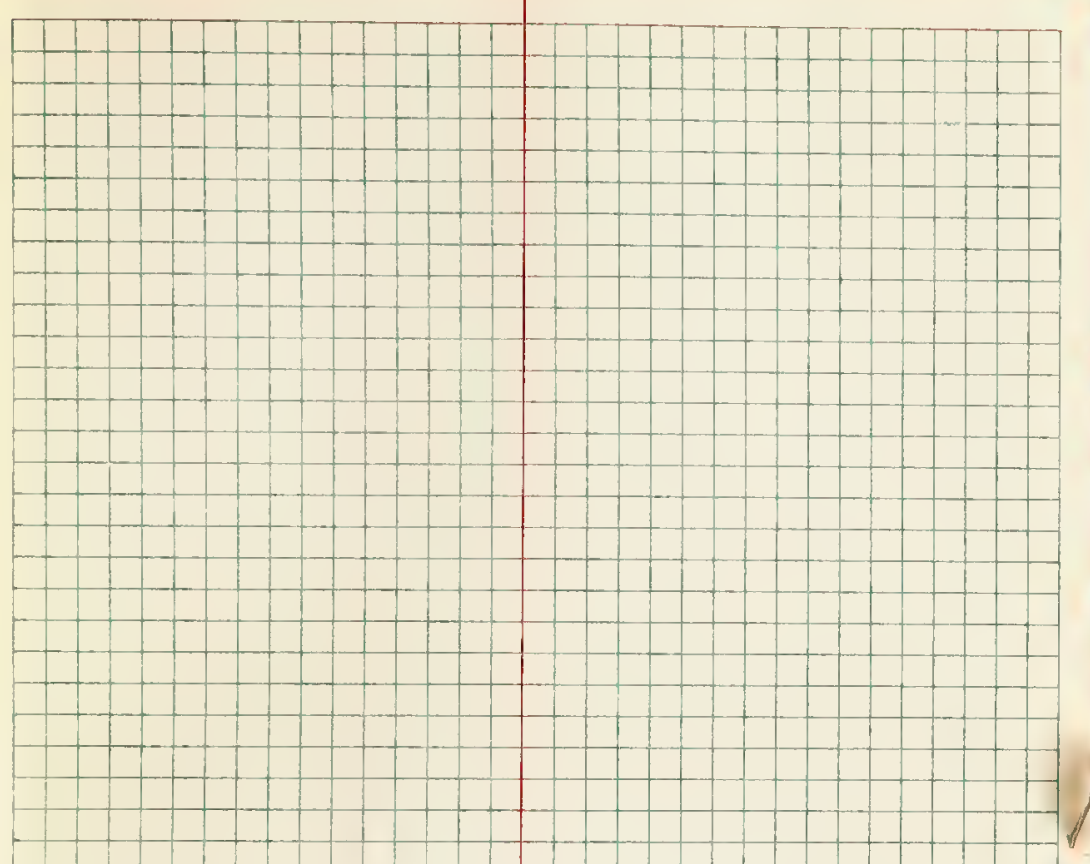




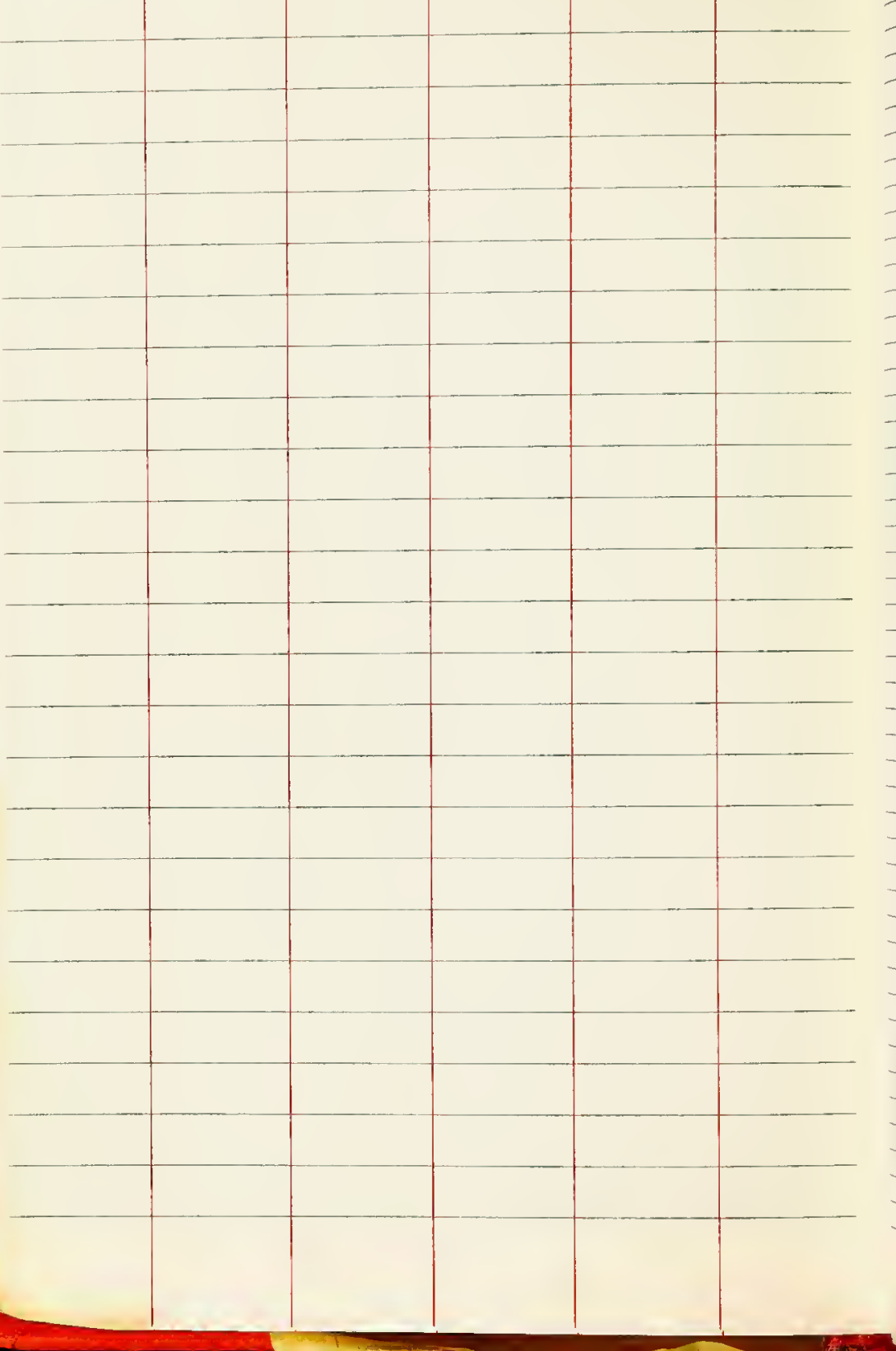





\section{CURVE AND REDUCTION TABLES}

\section{Published by Eugene Dietzgen Co.}

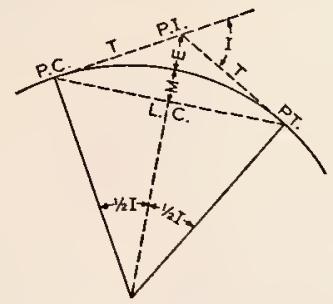

\section{CURVE FORMULAS}

1. Radius

$$
\text { : } \mathrm{R}=\frac{50}{\sin \mathrm{D} / 2}
$$

2. Degree of Curve: $\mathrm{D}=100 \frac{\mathrm{I}}{\mathrm{L}} \quad$ Also, $\sin \mathrm{D} / 2=\frac{50}{\mathrm{R}}$

3. Tangent : $\mathrm{T}=\mathrm{R} \tan 1 / 2 \mathrm{I} . \quad$ Also, $\mathrm{T}=\frac{\mathrm{T} \text { for } 1^{\circ} \text { curve }}{\mathrm{D}}+\mathrm{C}$.

4. Length of Curve: $\mathrm{L}=100 \frac{\mathrm{I}}{\mathrm{D}}$

5. Long Chord : L. C. $=2 \mathrm{R} \sin 1 / 2 \mathrm{I}$.

6. Middle Ordinate: $M=R(1-\cos 1 / 2 \mathrm{I})$

7. External : $\mathrm{E}=\frac{\mathrm{R}}{\cos 1 / 2 \mathrm{I}}-\mathrm{R}$. Also, $\mathrm{E}=\mathrm{T} \tan 1 / 4 \mathrm{I}$.

\section{EXPLANATION AND USE OF TABLES}

Given P.I. Sta. $83+40.7, I=45^{\circ} 20^{\prime}$ and $\mathrm{D}=6^{\circ} 30^{\prime}$ find:

Stations - P. C. $=$ P.I. - T. $T=\frac{T \text { for } 1^{\circ} \text { Curve }}{D}+$ C. From Tables V and VI $\mathrm{T}=\frac{2392.8}{6.5}+.197=368.32=3+68.32 . \quad$ Sta. P. C. $=83+40.7-(3+68.32)=79+72.38$ P. T. $=$ P. C. + L, and $\mathrm{L}=100 \frac{\mathrm{I}}{\mathrm{D}}=100 \frac{45.33}{6.5}=697.38$ Therefore, P. T. $=(79+72.38)$ $+(6+97.38)=86+69.76$.

Offsets-Tangent offsets vary (approximately) directly with $D$ and with the square of the distance. From Table III Tangent Offet for 100 feet $=5.669$ feet. Distance $=80-$ Sta. P. C. $=27.62$. Hence offset $=5.66 \times\left(\frac{27.62}{100}\right)^{2}=.432 \mathrm{ft}$. Also, square of any distance, divided by twice the radius equals (approximately) the distance from tangent to curve. Thus $(27.62)^{2} \div(2 \times 881.95)=.432 \mathrm{ft}$.

Deflectiong-Deflection angle $=1 / 2 \mathrm{D}$ for $100 \mathrm{ft}$., 1/4 D for $50 \mathrm{ft}$., etc. For " $\mathrm{X}$ " ft." Deflection Angle (in minutes) $=.3 \times X \times D$. For Sta. 80 of above curve Deflection Angle $=.3 \times 27.62 \times 6.5=53.86^{\prime}$. Also Deflection Angle $=d$ fll. for $1 \mathrm{ft}$. from Table III $\times \mathrm{X}=1.95$ $\times 27.62=53.86^{\prime}$. For Sta. 181 Deflection Angle $=53.86^{\prime}+\frac{6^{\circ} 30^{\prime}}{2}=4^{\circ} 8.86^{\prime}$.

Externals-From Table $\mathrm{V}$ for $1^{\circ}$ curve, with central angle of $45^{\circ} 20^{\prime}, E=479.6$. Therefore, for $6^{\circ} 30^{\prime}$ curve, $E=\frac{479.6}{6.5}+$ Correction from Table VI $=7.378+.039=7.417$. 


\section{General Equations and Useful Data}

\begin{tabular}{|c|c|c|}
\hline & $\begin{array}{c}\text { Symbol } \\
\text { on Equation }\end{array}$ & $\begin{array}{c}\text { Numerical } \\
\text { Value }\end{array}$ \\
\hline Area of circle $\ldots \ldots \ldots \ldots \ldots \ldots$ & $\frac{\pi \mathrm{d}^{2}}{4}$ & \\
\hline $\begin{array}{l}\text { Area of Sector of Circle (Length of } \\
\operatorname{arc}=1) \ldots \ldots \ldots\end{array}$ & $1 / 2 \mathrm{lr}$ & \\
\hline $\begin{array}{l}\text { Area of Segment of Parabola (app.) } \\
\quad(c=\text { chord; } m=\text { mid. ord. }) . . . .\end{array}$ & $2 / 3 \mathrm{~cm}$ & \\
\hline Area of Segment of Circle........ & $2 / 3 \mathrm{~cm}$ & \\
\hline Cubic Feet in 1 Cubic Meter..... & & 35.3145 \\
\hline Cubic Inches in 1 Imperial Gallon. & & 277.274 \\
\hline Cubic Inches in 1 U.S. Gallon.... & & 231 \\
\hline Feet in 1 Meter.............. & & 3.2808 \\
\hline Feet in 1 Mile $\ldots \ldots \ldots \ldots \ldots \ldots$ & & 5280 \\
\hline Gallons (Imperial) in 1 Cubic Foot. & & 6.2321 \\
\hline Gallons (U.S.) in 1 Cubie Foot.... & & 7.48 \\
\hline Miles in 1 Kilometer........... & & 0.6213 \\
\hline Pounds (Av.) in 1 Kilogram...... & & 2.2046 \\
\hline Ratio of Circumference to Diameter & $\pi$ & 3.14159 \\
\hline Reciprocal of $\pi \ldots \ldots \ldots \ldots \ldots$ & & 0.31831 \\
\hline Square Feet in 1 Acre.......... & & 43,560 \\
\hline Volume of Sphere.............. & $4 / 3 \pi r^{3}$ & \\
\hline
\end{tabular}

\section{Table I.-Minutes in Decimals of a Degree.}

\begin{tabular}{|c|c|c|c|c|c|c|c|c|c|c|c|}
\hline $\begin{array}{r}1^{\prime} \\
2 \\
3 \\
4 \\
5 \\
6 \\
7 \\
8 \\
9 \\
10\end{array}$ & $\begin{array}{l}.0167 \\
.0333 \\
.0500 \\
.0667 \\
.0833 \\
.1000 \\
.1167 \\
.1333 \\
.1500 \\
.1667\end{array}$ & $\begin{array}{l}11^{\prime} \\
12 \\
13 \\
14 \\
15 \\
16 \\
17 \\
18 \\
19 \\
20\end{array}$ & $\begin{array}{l}1533 \\
2000 \\
2167 \\
2333 \\
2500 \\
2667 \\
2933 \\
3000 \\
3167 \\
3333\end{array}$ & $\begin{array}{l}21 \\
22 \\
23 \\
24 \\
25 \\
26 \\
27 \\
28 \\
29 \\
30\end{array}$ & $\begin{array}{l}.3500 \\
.3667 \\
.3833 \\
4000 \\
4167 \\
4333 \\
4500 \\
4667 \\
4833 \\
5000\end{array}$ & $\begin{array}{l}31^{\prime} \\
32 \\
33 \\
34 \\
35 \\
36 \\
37 \\
38 \\
39 \\
40\end{array}$ & $\begin{array}{l}.5167 \\
.5333 \\
5500 \\
5667 \\
.5333 \\
6000 \\
.6167 \\
.6333 \\
.6500 \\
6667\end{array}$ & $\begin{array}{l}41^{\prime} \\
42 \\
43 \\
44 \\
45 \\
46 \\
47 \\
48 \\
49 \\
50\end{array}$ & $\begin{array}{l}.6833 \\
7000 \\
7167 \\
7333 \\
.7500 \\
.7667 \\
.7833 \\
.8000 \\
8167 \\
8333\end{array}$ & $\begin{array}{l}51^{\prime} \\
\mathbf{5 2} \\
\mathbf{5 3} \\
54 \\
55 \\
56 \\
57 \\
58 \\
59 \\
60\end{array}$ & $\begin{array}{r}.8500 \\
.8667 \\
.8833 \\
.9000 \\
.9167 \\
.9333 \\
.9500 \\
.9667 \\
.9833 \\
1.0000\end{array}$ \\
\hline
\end{tabular}

\section{Table II.-Inches in Decimals of a Foot.}

\begin{tabular}{|c|c|c|c|c|c|c|c|c|c|c|}
\hline $1 / 16$ & $3 / 2$ & $1 / 3$ & $3 / 60$ & $1 / 4$ & $3 / 6$ & $3 / 8$ & $1 / 2$ & $8 / 8$ & $3 / 4$ & $7 / 6$ \\
0052 & 0078 & 0104 & 0156 & 0208 & 0260 & 0313 & 0417 & .0521 & 0625 & .0729 \\
\hline \hline 1 & 2 & 3 & 4 & 5 & 6 & 7 & 8 & 9 & 10 & 11 \\
\hline 0833 & 1667 & 2500 & 3333 & 4167 & 5000 & 5833 & 6667 & 7500 & .8333 & 9167 \\
\hline
\end{tabular}


Table III.-Radii, Ordinates and Deflections

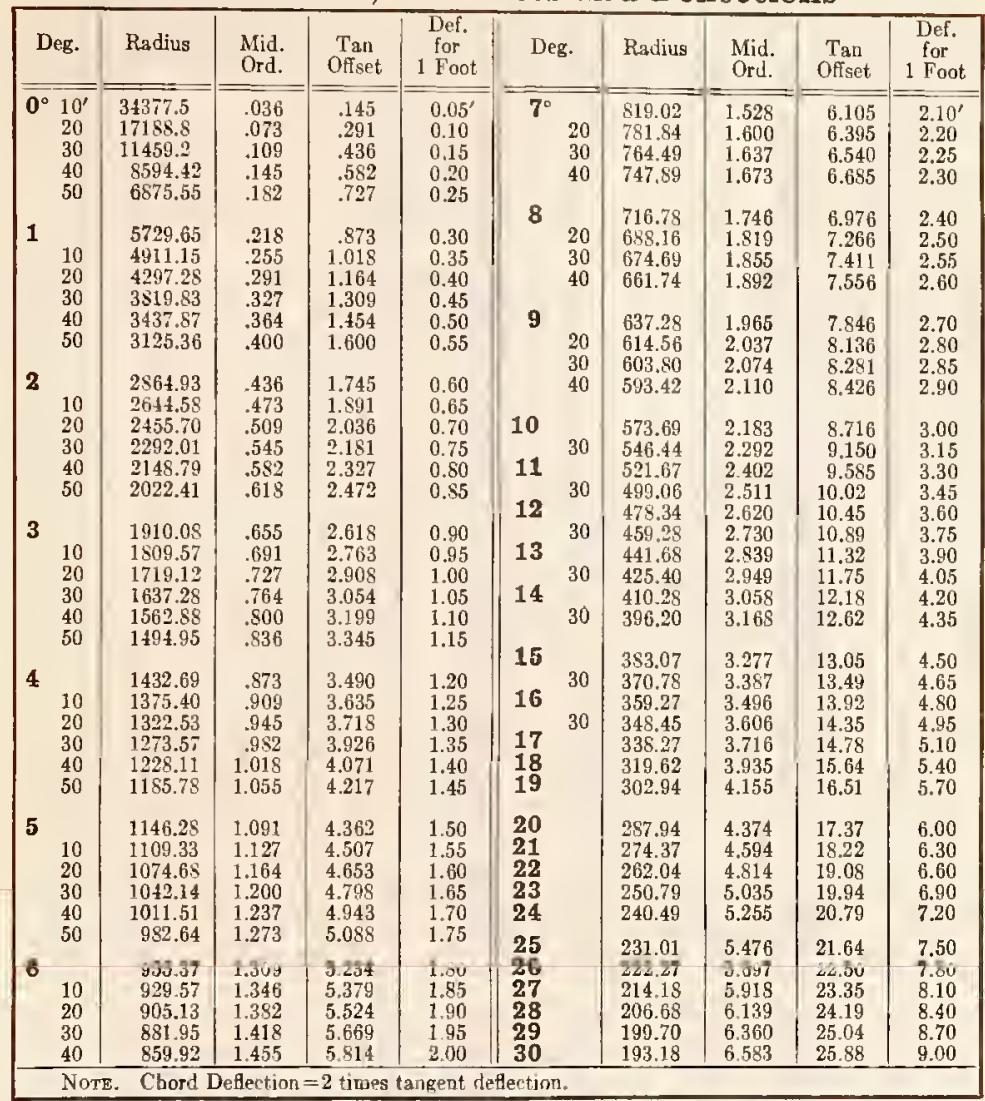

\section{Table IV.-Deflections for Sub Chords for Short Radius Curves}

\begin{tabular}{|c|c|c|c|c|c|c|}
\hline \multirow{2}{*}{$\begin{array}{l}\text { Degree } \\
\text { of } \\
\text { Curve }\end{array}$} & \multirow{2}{*}{ Radius } & \multicolumn{4}{|c|}{$\begin{array}{l}\text { Deflection Angles for } \\
\text { Sub Chords of Various Lengths }\end{array}$} & \multirow{2}{*}{$\begin{array}{c}\text { Length of } \\
\text { Arc for } \\
100 \mathrm{Ft} \text {. Chord }\end{array}$} \\
\hline & & $12.5 \mathrm{Ft}$. & $15 \mathrm{Ft}$. & $20 \mathrm{Ft}$. & $25 \mathrm{Ft}$. & \\
\hline $\begin{array}{l}30^{\circ} \\
32^{\circ} \\
34^{\circ} \\
36^{\circ} \\
38^{\circ} \\
40^{\circ} \\
42^{\circ} \\
44^{\circ} \\
46^{\circ} \\
48^{\circ} \\
50^{\circ} \\
52^{\circ} \\
54^{\circ} \\
56^{\circ} \\
58^{\circ} \\
\mathbf{6} 0^{\circ}\end{array}$ & $\begin{array}{l}193.18 \\
181.39 \\
171.01 \\
161.80 \\
153.58 \\
146.19 \\
139.52 \\
133.47 \\
127.97 \\
122.92 \\
118.31 \\
114.06 \\
110.11 \\
106.50 \\
103.14 \\
100.00\end{array}$ & $\begin{array}{ll}1^{\circ} & 51^{\prime} \\
1^{\circ} & 59^{\prime} \\
2^{\circ} & 06^{\prime} \\
2^{\circ} & 13^{\prime} \\
2^{\circ} & 20^{\prime} \\
2^{\circ} & 27^{\prime} \\
2^{\circ} & 34^{\prime} \\
2^{\circ} & 41^{\prime} \\
2^{\circ} & 48^{\prime} \\
2^{\circ} & 55^{\prime} \\
3^{\circ} & 02^{\prime} \\
3^{\circ} & 09^{\prime} \\
3^{\circ} & 16^{\prime} \\
3^{\circ} & 22^{\prime} \\
3^{\circ} & 29^{\prime} \\
3^{\circ} & 35^{\prime}\end{array}$ & $\begin{array}{l}2^{\circ} 17^{\prime} \\
2^{\circ} 25^{\prime} \\
2^{\circ} 33^{\prime} \\
2^{\circ} 41^{\prime} \\
2^{\circ} 49^{\prime} \\
2^{\circ} 57^{\prime} \\
3^{\circ} 05^{\prime} \\
3^{\circ} 13^{\prime} \\
3^{\circ} 21^{\prime} \\
3^{\circ} 29^{\prime} \\
3^{\circ} 38^{\prime} \\
3^{\circ} 46^{\prime} \\
3^{\circ} 54^{\prime} \\
4^{\circ} 02^{\prime} \\
4^{\circ} 10^{\prime} \\
4^{\circ} 18^{\prime}\end{array}$ & $\begin{array}{l}2^{\circ} 58^{\prime} \\
3^{\circ} 10^{\prime} \\
3^{\circ} 21^{\prime} \\
3^{\circ} 33^{\prime} \\
3^{\circ} 44^{\prime} \\
3^{\circ} 55^{\prime} \\
4^{\circ} 07^{\prime} \\
4^{\circ} 18^{\prime} \\
4^{\circ} 29^{\prime} \\
4^{\circ} 40^{\prime} \\
4^{\circ} 51^{\prime} \\
6^{\circ} 02^{\prime} \\
5^{\circ} 13^{\prime} \\
5^{\circ} 23^{\prime} \\
5^{\circ} 34^{\prime} \\
5^{\circ} 44^{\prime}\end{array}$ & $\begin{array}{l}3^{\circ} 43^{\prime} \\
3^{\circ} 58^{\prime} \\
4^{\circ} 12^{\prime} \\
4^{\circ} 26^{\prime} \\
4^{\circ} 40^{\prime} \\
4^{\circ} 54^{\prime} \\
5^{\circ} 08^{\prime} \\
5^{\circ} 22^{\prime} \\
5^{\circ} 36^{\prime} \\
5^{\circ} 50^{\prime} \\
6^{\circ} 04^{\prime} \\
6^{\circ} 17^{\prime} \\
6^{\circ} 31^{\prime} \\
6^{\circ} 44^{\prime} \\
6^{\circ} 57^{\prime} \\
7^{\circ} 11^{\prime}\end{array}$ & $\begin{array}{l}101.15 \\
101.33 \\
101.48 \\
101.66 \\
101.85 \\
102.06 \\
102.29 \\
102.53 \\
102.76 \\
103.00 \\
103.24 \\
103.54 \\
103.84 \\
104.14 \\
104.43 \\
104.72\end{array}$ \\
\hline
\end{tabular}


Table V.-Tangents and Externals to a $1^{\circ}$ Curve.

\begin{tabular}{|c|c|c|c|c|c|c|c|c|}
\hline $\begin{array}{l}\text { Central } \\
\text { Angle }\end{array}$ & Tangent & External & $\begin{array}{c}\text { Central } \\
\text { Angle }\end{array}$ & Tangent & External & $\begin{array}{c}\text { Central } \\
\text { Angle }\end{array}$ & Tangent & External \\
\hline $\begin{array}{c}1^{\circ} \\
10^{\prime} \\
20 \\
30 \\
40 \\
50\end{array}$ & $\begin{array}{l}50.00 \\
58.34 \\
66.67 \\
75.01 \\
83.34 \\
91.68\end{array}$ & $\begin{array}{l}.22 \\
.30 \\
.39 \\
.49 \\
.61 \\
.73\end{array}$ & $\begin{array}{r}11^{\circ} \\
10 \\
20 \\
30 \\
40 \\
50\end{array}$ & $\begin{array}{l}551.70 \\
560.11 \\
568.53 \\
576.95 \\
585.36 \\
593.79\end{array}$ & $\begin{array}{l}26.50 \\
27.31 \\
28.14 \\
28.97 \\
29.82 \\
30.68\end{array}$ & $\begin{array}{c}21^{\circ} \\
10^{\prime} \\
20 \\
30 \\
40 \\
50\end{array}$ & $\begin{array}{l}1061.9 \\
1070.6 \\
1079.2 \\
1087.8 \\
1096.4 \\
1105.1\end{array}$ & $\begin{array}{r}97.57 \\
99.16 \\
100.75 \\
102.35 \\
103.97 \\
105.60\end{array}$ \\
\hline $\begin{array}{l}10 \\
20 \\
30 \\
40 \\
50\end{array}$ & $\begin{array}{l}100.01 \\
108.35 \\
116.68 \\
125.02 \\
133.36 \\
141.70\end{array}$ & $\begin{array}{l}.87 \\
1.02 \\
1.19 \\
1.36 \\
1.55 \\
1.75\end{array}$ & $12 \begin{array}{r}10 \\
20 \\
30 \\
40 \\
50\end{array}$ & $\begin{array}{l}602.21 \\
610.64 \\
619.07 \\
627.50 \\
635.93 \\
644.37\end{array}$ & $\begin{array}{l}31.56 \\
32.45 \\
33.35 \\
34.26 \\
35.18 \\
36.12\end{array}$ & $22 \begin{array}{r}10 \\
20 \\
30 \\
40 \\
50\end{array}$ & $\begin{array}{l}1113.7 \\
1122.4 \\
1131.0 \\
1139.7 \\
1148.4 \\
1157.0\end{array}$ & $\begin{array}{l}107.24 \\
108.90 \\
110.57 \\
112.25 \\
113.95 \\
115.66\end{array}$ \\
\hline $\begin{array}{l}10 \\
20 \\
30 \\
40 \\
50\end{array}$ & $\begin{array}{l}150.04 \\
158.38 \\
166.72 \\
175.06 \\
183.40 \\
191.74\end{array}$ & $\begin{array}{l}1.96 \\
2.19 \\
2.43 \\
2.67 \\
2.93 \\
3.21\end{array}$ & $13 \begin{array}{r}10 \\
20 \\
30 \\
40 \\
50\end{array}$ & $\begin{array}{l}652.81 \\
661.25 \\
669.70 \\
678.15 \\
686.60 \\
695.06\end{array}$ & $\begin{array}{l}37.07 \\
38.03 \\
39.01 \\
39.99 \\
40.99 \\
42.00\end{array}$ & $\begin{array}{r}23 \\
10 \\
20 \\
30 \\
40 \\
50\end{array}$ & $\begin{array}{l}1165.7 \\
1174.4 \\
1183.1 \\
1191.8 \\
1200.5 \\
1209.2\end{array}$ & $\begin{array}{l}117.38 \\
119.12 \\
120.87 \\
122.63 \\
124.41 \\
126.20\end{array}$ \\
\hline $\begin{array}{l}10 \\
20 \\
30 \\
40 \\
50\end{array}$ & $\begin{array}{l}200.08 \\
208.43 \\
216.77 \\
225.12 \\
233.47 \\
241.81\end{array}$ & $\begin{array}{l}3.49 \\
3.79 \\
4.10 \\
4.42 \\
4.76 \\
5.10\end{array}$ & $14 \begin{array}{r}10 \\
20 \\
30 \\
40 \\
50\end{array}$ & $\begin{array}{l}703.51 \\
711.97 \\
720.44 \\
728.90 \\
737.37 \\
745.85\end{array}$ & $\begin{array}{l}43.03 \\
44.07 \\
45.12 \\
46.18 \\
47.25 \\
48.34\end{array}$ & $24 \begin{array}{r} \\
10 \\
20 \\
30 \\
40 \\
50\end{array}$ & $\begin{array}{l}1217.9 \\
1226.6 \\
1235.3 \\
1244.0 \\
1252.8 \\
1261.5\end{array}$ & $\begin{array}{l}128.00 \\
129.82 \\
131.65 \\
133.50 \\
135.35 \\
137.23\end{array}$ \\
\hline $\begin{array}{l}10 \\
20 \\
30 \\
40 \\
50\end{array}$ & $\begin{array}{l}250.16 \\
258.51 \\
266.86 \\
275.21 \\
283.57 \\
291.92\end{array}$ & $\begin{array}{l}5.46 \\
5.83 \\
6.21 \\
6.61 \\
7.01 \\
7.43\end{array}$ & $15 \begin{array}{r}10 \\
20 \\
30 \\
40 \\
50\end{array}$ & $\begin{array}{l}754.32 \\
762.80 \\
771.29 \\
779.77 \\
788.26 \\
796.75\end{array}$ & $\begin{array}{l}49.44 \\
50.55 \\
51.68 \\
52.89 \\
53.97 \\
55.13\end{array}$ & $\begin{array}{l}25 \\
10 \\
20 \\
30 \\
40 \\
50\end{array}$ & $\begin{array}{l}1270.2 \\
1279.0 \\
1287.7 \\
1296.5 \\
1305.3 \\
1314.0\end{array}$ & $\begin{array}{l}139.11 \\
141.01 \\
142.93 \\
144.85 \\
146.79 \\
148.75\end{array}$ \\
\hline $\begin{array}{l}10 \\
20 \\
30 \\
40 \\
50\end{array}$ & $\begin{array}{l}300.28 \\
308.64 \\
316.99 \\
325.35 \\
333.71 \\
342.08\end{array}$ & $\begin{array}{r}7.86 \\
8.31 \\
8.76 \\
9.23 \\
9.71 \\
10.20\end{array}$ & $16 \begin{array}{r}10 \\
10 \\
20 \\
30 \\
40 \\
50\end{array}$ & $\begin{array}{l}805.25 \\
813.75 \\
822.25 \\
830.76 \\
839.27 \\
847.78\end{array}$ & $\begin{array}{l}56.31 \\
57.50 \\
58.70 \\
59.91 \\
61.14 \\
62.38\end{array}$ & $26 \begin{array}{l}10 \\
20 \\
30 \\
40 \\
50\end{array}$ & $\begin{array}{l}1322.8 \\
1331.6 \\
1340.4 \\
1349.2 \\
1358.0 \\
1366.8\end{array}$ & $\begin{array}{l}150.71 \\
152.69 \\
154.69 \\
156.70 \\
158.72 \\
160.76\end{array}$ \\
\hline $\begin{array}{l}10 \\
20 \\
30 \\
40 \\
50\end{array}$ & $\begin{array}{l}350.44 \\
358.81 \\
367.17 \\
375.54 \\
383.91 \\
392.28\end{array}$ & $\begin{array}{l}10.71 \\
11.22 \\
11.75 \\
12.29 \\
12.85 \\
13.41\end{array}$ & $\begin{array}{r}17 \\
10 \\
20 \\
30 \\
40 \\
50\end{array}$ & $\begin{array}{l}856.30 \\
864.82 \\
873.35 \\
881.88 \\
890.41 \\
898.95\end{array}$ & $\begin{array}{l}63.63 \\
64.90 \\
66.18 \\
67.47 \\
68.77 \\
70.09\end{array}$ & $\begin{array}{r}27 \\
10 \\
20 \\
30 \\
40 \\
50\end{array}$ & $\begin{array}{l}1375.6 \\
1384.4 \\
1393.2 \\
1402.0 \\
1410.9 \\
1419.7\end{array}$ & $\begin{array}{l}162.81 \\
164.86 \\
166.95 \\
169.04 \\
171.15 \\
173.27\end{array}$ \\
\hline $\begin{array}{l}10 \\
20 \\
30 \\
40 \\
50\end{array}$ & $\begin{array}{l}400.66 \\
409.03 \\
417.41 \\
425.79 \\
434.17 \\
442.55\end{array}$ & $\begin{array}{l}13.99 \\
14.58 \\
15.18 \\
15.80 \\
16.43 \\
17.07\end{array}$ & $\begin{array}{l}18 \\
10 \\
20 \\
30 \\
40 \\
50\end{array}$ & $\begin{array}{l}907.49 \\
916.03 \\
924.58 \\
933.13 \\
941.69 \\
950.25\end{array}$ & $\begin{array}{l}71.42 \\
72.76 \\
74.12 \\
75.49 \\
76.86 \\
78.26\end{array}$ & $\begin{array}{r}28 \\
10 \\
20 \\
30 \\
40 \\
50\end{array}$ & $\begin{array}{l}1428.6 \\
1437.4 \\
1446.3 \\
1455.1 \\
1464.0 \\
1472.9\end{array}$ & $\begin{array}{l}175.41 \\
177.55 \\
179.72 \\
181.89 \\
184.08 \\
186.29\end{array}$ \\
\hline $\begin{array}{l}10 \\
20 \\
30 \\
40 \\
50\end{array}$ & $\begin{array}{l}450.93 \\
459.32 \\
467.71 \\
476.10 \\
484.49 \\
492.88\end{array}$ & $\begin{array}{l}17.72 \\
18.38 \\
19.06 \\
19.75 \\
20.45 \\
21.16\end{array}$ & $19 \begin{array}{r}10 \\
20 \\
30 \\
40 \\
50\end{array}$ & $\begin{array}{r}958.81 \\
967.38 \\
975.96 \\
984.53 \\
993.12 \\
1001.7\end{array}$ & $\begin{array}{l}79.67 \\
81.09 \\
82.53 \\
83.97 \\
85.43 \\
86.90\end{array}$ & $29 \begin{array}{r}10 \\
20 \\
30 \\
40 \\
50\end{array}$ & $\begin{array}{l}1481.8 \\
1490.7 \\
1499.6 \\
1508.5 \\
1517.4 \\
1526.3\end{array}$ & $\begin{array}{l}188.51 \\
190.74 \\
192.99 \\
195.25 \\
197.53 \\
199.82\end{array}$ \\
\hline $\begin{array}{r}10 \\
10 \\
20 \\
30 \\
40 \\
50\end{array}$ & $\begin{array}{l}501.28 \\
509.68 \\
51808 \\
526.48 \\
534.89 \\
543.29\end{array}$ & $\begin{array}{l}21.89 \\
22.62 \\
23.38 \\
24.14 \\
24.91 \\
25.70\end{array}$ & $\begin{array}{r}20 \\
10 \\
20 \\
30 \\
40 \\
50\end{array}$ & $\begin{array}{l}1010.3 \\
1018.9 \\
1027.5 \\
1036.1 \\
1044.7 \\
1053.3\end{array}$ & $\begin{array}{l}88.39 \\
89.89 \\
91.40 \\
92.92 \\
94.46 \\
96.01\end{array}$ & $\begin{array}{r}30 \\
10 \\
20 \\
30 \\
40 \\
50\end{array}$ & $\begin{array}{l}1535.3 \\
1544.2 \\
1553.1 \\
1562.1 \\
1571.0 \\
1580.0\end{array}$ & $\begin{array}{l}202.12 \\
204.44 \\
206.77 \\
209.12 \\
211.48 \\
213.86\end{array}$ \\
\hline
\end{tabular}


Table V.-Tangents and Externals to a $1^{\circ}$ Curve.

\begin{tabular}{|c|c|c|c|c|c|c|c|c|}
\hline $\begin{array}{c}\text { Central } \\
\text { Angle }\end{array}$ & Tangent & External & $\begin{array}{c}\text { Central } \\
\text { Ansle }\end{array}$ & Tangent & External & $\begin{array}{c}\text { Central } \\
\text { Angle }\end{array}$ & Tangent & External \\
\hline $\begin{array}{c}31^{\circ} \\
10^{\prime} \\
20 \\
30 \\
40 \\
50\end{array}$ & $\begin{array}{l}1589.0 \\
1598.0 \\
1606.9 \\
1615.9 \\
1624.9 \\
1633.9\end{array}$ & $\begin{array}{l}216.3 \\
218.7 \\
221.1 \\
223.5 \\
226.0 \\
228.4\end{array}$ & $\begin{array}{r}41^{\circ} \\
10 \\
20 \\
30 \\
40 \\
50\end{array}$ & $\begin{array}{l}2142.2 \\
2151.7 \\
2161.2 \\
2170.8 \\
2180.3 \\
2189.9\end{array}$ & $\begin{array}{l}387.4 \\
390.7 \\
394.1 \\
397.4 \\
400.8 \\
404.2\end{array}$ & $\begin{array}{r}51^{\circ} \\
10 \\
20 \\
30 \\
40 \\
50\end{array}$ & $\begin{array}{l}2732.9 \\
2743.1 \\
2753.4 \\
2763.7 \\
2773.9 \\
2784.2\end{array}$ & $\begin{array}{l}618.4 \\
622.8 \\
627.2 \\
631.7 \\
636.2 \\
640.7\end{array}$ \\
\hline $\begin{array}{l}32 \\
10 \\
20 \\
30 \\
40 \\
50\end{array}$ & $\begin{array}{l}1643.0 \\
1652.0 \\
1661.0 \\
1670.0 \\
1679.1 \\
1688.1\end{array}$ & $\begin{array}{l}230.9 \\
233.4 \\
235.9 \\
238.4 \\
241.0 \\
243.5\end{array}$ & $\begin{array}{r}42 \\
10 \\
20 \\
30 \\
40 \\
50\end{array}$ & $\begin{array}{l}2199.4 \\
2209.0 \\
2218.6 \\
2228.1 \\
2237.7 \\
2247.3\end{array}$ & $\begin{array}{l}407.6 \\
411.1 \\
414.5 \\
418.0 \\
421.4 \\
425.0\end{array}$ & $\begin{array}{ll}52 & \\
10 \\
20 \\
30 \\
40 \\
50\end{array}$ & $\begin{array}{l}2794.5 \\
2804.9 \\
2815.2 \\
2825.6 \\
2835.9 \\
2846.3\end{array}$ & $\begin{array}{l}645.2 \\
649.7 \\
654.3 \\
658.8 \\
663.4 \\
668.0\end{array}$ \\
\hline $\begin{array}{r}33 \\
10 \\
20 \\
30 \\
40 \\
50\end{array}$ & $\begin{array}{l}1697.2 \\
1706.3 \\
1715.3 \\
1724.4 \\
1733.5 \\
1742.6\end{array}$ & $\begin{array}{l}246.1 \\
248.7 \\
251.3 \\
253.9 \\
256.5 \\
259.1\end{array}$ & $\begin{array}{r}43 \\
10 \\
20 \\
30 \\
40 \\
50\end{array}$ & $\begin{array}{l}2257.0 \\
2266.6 \\
2276.2 \\
2285.9 \\
2295.6 \\
2305.2\end{array}$ & $\begin{array}{l}428.5 \\
432.0 \\
435.6 \\
439.2 \\
442.8 \\
446.4\end{array}$ & $\begin{array}{r}53 \\
10 \\
20 \\
30 \\
40 \\
50\end{array}$ & $\begin{array}{l}2856.7 \\
2867.1 \\
2877.5 \\
2888.0 \\
2898.4 \\
2908.9\end{array}$ & $\begin{array}{l}672.7 \\
677.3 \\
682.0 \\
686.7 \\
691.4 \\
696.1\end{array}$ \\
\hline $\begin{array}{l}34 \\
10 \\
20 \\
30 \\
40 \\
50\end{array}$ & $\begin{array}{l}1751.7 \\
1760.8 \\
1770.0 \\
1779.1 \\
1788.2 \\
1797.4\end{array}$ & $\begin{array}{l}261.8 \\
264.5 \\
267.2 \\
269.9 \\
272.6 \\
275.3\end{array}$ & $\begin{array}{r}44 \\
10 \\
20 \\
30 \\
40 \\
50\end{array}$ & $\begin{array}{l}2314.9 \\
2324.6 \\
2334.3 \\
2344.1 \\
2353.8 \\
2363.5\end{array}$ & $\begin{array}{l}450.0 \\
453.6 \\
457.3 \\
461.0 \\
464.6 \\
468.4\end{array}$ & $\begin{array}{rr}54 & \\
10 \\
20 \\
30 \\
40 \\
50\end{array}$ & $\begin{array}{l}2919.4 \\
2929.9 \\
2940.4 \\
2951.0 \\
2961.5 \\
2972.1\end{array}$ & $\begin{array}{l}700.9 \\
705.7 \\
710.5 \\
715.3 \\
720.1 \\
725.0\end{array}$ \\
\hline $\begin{array}{l}35 \\
10 \\
20 \\
30 \\
40 \\
50\end{array}$ & $\begin{array}{l}1806.6 \\
1815.7 \\
1824.9 \\
1834.1 \\
1843.3 \\
1852.5\end{array}$ & $\begin{array}{l}278.1 \\
280.8 \\
283.6 \\
286.4 \\
289.2 \\
292.0\end{array}$ & $45 \begin{array}{r} \\
10 \\
20 \\
30 \\
40 \\
50\end{array}$ & $\begin{array}{l}2373.3 \\
2383.1 \\
2392.8 \\
2402.6 \\
2412.4 \\
2422.3\end{array}$ & $\begin{array}{l}472.1 \\
475.8 \\
479.6 \\
483.8 \\
487.2 \\
491.0\end{array}$ & $\begin{array}{ll}55 & \\
10 \\
20 \\
30 \\
40 \\
50\end{array}$ & $\begin{array}{l}2982.7 \\
2993.3 \\
3003.9 \\
3014.5 \\
3025.2 \\
3035.8\end{array}$ & $\begin{array}{l}729.9 \\
734.8 \\
739.7 \\
744.6 \\
749.6 \\
754.6\end{array}$ \\
\hline $\begin{array}{r}36 \\
10 \\
20 \\
30 \\
40 \\
50\end{array}$ & $\begin{array}{l}1861.7 \\
1870.9 \\
1880.1 \\
1889.4 \\
1898.6 \\
1907.9\end{array}$ & $\begin{array}{l}294.9 \\
297.7 \\
300.6 \\
303.5 \\
306.4 \\
309.3\end{array}$ & $\begin{array}{r}46 \\
10 \\
20 \\
30 \\
40 \\
50\end{array}$ & $\begin{array}{l}2432.1 \\
2441.9 \\
2451.8 \\
2461.7 \\
2471.5 \\
2481.4\end{array}$ & $\begin{array}{l}494.8 \\
498.7 \\
502.5 \\
506.4 \\
510.3 \\
514.3\end{array}$ & $\begin{array}{r}56 \\
10 \\
20 \\
30 \\
40 \\
50\end{array}$ & $\begin{array}{l}3046.5 \\
3057.2 \\
3067.9 \\
3078.7 \\
3089.4 \\
3100.2\end{array}$ & $\begin{array}{l}759.6 \\
764.6 \\
769.7 \\
774.7 \\
779.8 \\
784.9\end{array}$ \\
\hline $\begin{array}{r}37 \\
10 \\
20 \\
30 \\
40 \\
50\end{array}$ & $\begin{array}{l}1917.1 \\
1926.4 \\
1935.7 \\
1945.0 \\
1954.3 \\
1963.6\end{array}$ & $\begin{array}{l}312.2 \\
315.2 \\
318.1 \\
321.1 \\
324.1 \\
327.1\end{array}$ & $\begin{array}{r}47 \\
10 \\
20 \\
30 \\
40 \\
50\end{array}$ & $\begin{array}{l}2491.3 \\
2501.2 \\
2511.2 \\
2521.1 \\
2531.1 \\
2541.0\end{array}$ & $\begin{array}{l}518.2 \\
522.2 \\
526.1 \\
530.1 \\
534.2 \\
538.2\end{array}$ & $\begin{array}{r}57 \\
10 \\
20 \\
30 \\
40 \\
50\end{array}$ & $\begin{array}{l}3110.9 \\
3121.7 \\
3132.6 \\
3143.4 \\
3154.2 \\
3165.1\end{array}$ & $\begin{array}{l}790.1 \\
795.2 \\
800.4 \\
805.6 \\
810.9 \\
816.1\end{array}$ \\
\hline $\begin{array}{r}38 \\
10 \\
20 \\
30 \\
40 \\
50\end{array}$ & $\begin{array}{l}1972.9 \\
1982.2 \\
1991.5 \\
2000.9 \\
2010.2 \\
2019.6\end{array}$ & $\begin{array}{l}330.2 \\
333.2 \\
336.3 \\
339.3 \\
342.4 \\
345.5\end{array}$ & $\begin{array}{r}48 \\
10 \\
20 \\
30 \\
40 \\
50\end{array}$ & $\begin{array}{l}2551.0 \\
2561.0 \\
2571.0 \\
2581.0 \\
2591.0 \\
2601.1\end{array}$ & $\begin{array}{l}542.2 \\
546.3 \\
550.4 \\
554.5 \\
558.6 \\
562.8\end{array}$ & $\begin{array}{r}58 \\
10 \\
20 \\
30 \\
40 \\
50\end{array}$ & $\begin{array}{l}3176.0 \\
3186.9 \\
3197.8 \\
3208.8 \\
3219.7 \\
3230.7\end{array}$ & $\begin{array}{l}821.4 \\
826.7 \\
832.0 \\
837.3 \\
842.7 \\
848.1\end{array}$ \\
\hline $\begin{array}{r}39 \\
10 \\
20 \\
30 \\
40 \\
50\end{array}$ & $\begin{array}{l}2029.0 \\
2038.4 \\
2047.8 \\
2057.2 \\
2066.6 \\
2076.0\end{array}$ & $\begin{array}{l}348.6 \\
351.8 \\
354.9 \\
358.1 \\
361.3 \\
364.5\end{array}$ & $\begin{array}{r}49 \\
10 \\
20 \\
30 \\
40 \\
50\end{array}$ & $\begin{array}{l}2611.2 \\
2621.2 \\
2631.3 \\
2641.4 \\
2651.5 \\
2661.6\end{array}$ & $\begin{array}{l}566.9 \\
571.1 \\
575.3 \\
579.5 \\
583.8 \\
588.0\end{array}$ & $\begin{array}{r}59 \\
10 \\
20 \\
30 \\
40 \\
50\end{array}$ & $\begin{array}{l}3241.7 \\
3252.7 \\
3263.7 \\
3274.8 \\
3285.8 \\
3296.9\end{array}$ & $\begin{array}{l}853.5 \\
858.9 \\
864.3 \\
869.8 \\
875.3 \\
880.8\end{array}$ \\
\hline $\begin{array}{r}40 \\
10 \\
20 \\
30 \\
40 \\
.50\end{array}$ & $\begin{array}{l}2085.4 \\
2094.9 \\
2104.3 \\
2113.8 \\
2123.3 \\
2132.7\end{array}$ & $\begin{array}{l}367.7 \\
371.0 \\
374.2 \\
377.5 \\
380.8 \\
384.1\end{array}$ & $\begin{array}{r}50 \\
10 \\
20 \\
30 \\
40 \\
50\end{array}$ & $\begin{array}{l}2671.8 \\
2681.9 \\
2692.1 \\
2702.3 \\
2712.5 \\
2722.7\end{array}$ & $\begin{array}{l}592.3 \\
596.6 \\
600.9 \\
605.3 \\
609.6 \\
614.0\end{array}$ & $\begin{array}{r}60 \\
10 \\
20 \\
30 \\
40 \\
50\end{array}$ & $\begin{array}{l}3308.0 \\
3319.1 \\
3330.3 \\
3341.4 \\
3352.6 \\
3363.8\end{array}$ & $\begin{array}{l}886.4 \\
892.0 \\
897.5 \\
903.2 \\
908.8 \\
914.5\end{array}$ \\
\hline
\end{tabular}


Table V.-Tangents and Externals to a $1^{\circ}$ Curve.

\begin{tabular}{|c|c|c|c|c|c|c|c|c|}
\hline $\begin{array}{c}\text { Central } \\
\text { Angle }\end{array}$ & Tangent & External & $\begin{array}{c}\text { Central } \\
\text { Angle }\end{array}$ & Tangent & External & $\begin{array}{c}\text { Central } \\
\text { Angle }\end{array}$ & Tangent & External \\
\hline $\begin{array}{c}61^{\circ} \\
10^{\prime} \\
20 \\
30 \\
40 \\
50\end{array}$ & $\begin{array}{l}3375.0 \\
3386.3 \\
3397.5 \\
3408.8 \\
3420.1 \\
3431.4\end{array}$ & $\begin{array}{l}920.2 \\
925.9 \\
931.6 \\
937.3 \\
943.1 \\
948.9\end{array}$ & $\begin{array}{c}71^{\circ} \\
10^{\prime} \\
20 \\
30 \\
40 \\
50\end{array}$ & $\begin{array}{l}4086.9 \\
4099.5 \\
4112.1 \\
4124.8 \\
4137.4 \\
4150.1\end{array}$ & $\begin{array}{l}1308.2 \\
1315.6 \\
1322.9 \\
1330.3 \\
1337.7 \\
1345.1\end{array}$ & $\begin{array}{c}81^{\circ} \\
10^{\prime} \\
20 \\
30 \\
40 \\
50\end{array}$ & $\begin{array}{l}4893.6 \\
4908.0 \\
4922.5 \\
4937.0 \\
4951.5 \\
4966.1\end{array}$ & $\begin{array}{l}1805.3 \\
1814.7 \\
1824.1 \\
1833.6 \\
1843.1 \\
1852.6\end{array}$ \\
\hline $\begin{array}{r}62 \\
10 \\
20 \\
30 \\
40 \\
50\end{array}$ & $\begin{array}{l}3442.7 \\
3454.1 \\
3465.4 \\
3476.8 \\
3488.3 \\
3499.7\end{array}$ & $\begin{array}{l}954.8 \\
960.6 \\
966.5 \\
972.4 \\
978.3 \\
984.3\end{array}$ & $\begin{array}{r}72 \\
10 \\
20 \\
30 \\
40 \\
50\end{array}$ & $\begin{array}{l}4162.8 \\
4175.6 \\
4188.5 \\
4201.2 \\
4214.0 \\
4226.8\end{array}$ & $\begin{array}{l}1352.6 \\
1360.1 \\
1367.6 \\
1375.2 \\
1382.8 \\
1390.4\end{array}$ & $\begin{array}{l}82 \\
10 \\
20 \\
30 \\
40 \\
50\end{array}$ & $\begin{array}{l}4980.7 \\
4995.4 \\
5010.0 \\
5024.8 \\
5039.5 \\
5054.3\end{array}$ & $\begin{array}{l}1862.2 \\
1871.8 \\
1881.5 \\
1891.2 \\
1900.9 \\
1910.7\end{array}$ \\
\hline $\begin{array}{r}63 \\
10 \\
20 \\
30 \\
40 \\
50\end{array}$ & $\begin{array}{l}3511.1 \\
3522.6 \\
3534.1 \\
3545.6 \\
3557.2 \\
3568.7\end{array}$ & $\begin{array}{r}990.2 \\
996.2 \\
1002.3 \\
1008.3 \\
1014.4 \\
1020.5\end{array}$ & $\begin{array}{r}73 \\
10 \\
20 \\
30 \\
40 \\
50\end{array}$ & $\begin{array}{l}4239.7 \\
4252.6 \\
4265.6 \\
4278.5 \\
4291.5 \\
4304.6\end{array}$ & $\begin{array}{l}1398.0 \\
1405.7 \\
1413.5 \\
1421.2 \\
1429.0 \\
1436.8\end{array}$ & $\begin{array}{r}83 \\
10 \\
20 \\
30 \\
40 \\
50\end{array}$ & $\begin{array}{l}5069.2 \\
5084.0 \\
5099.0 \\
5113.9 \\
5128.9 \\
5143.9\end{array}$ & $\begin{array}{l}1920.5 \\
1930.4 \\
1940.3 \\
1950.3 \\
1960.2 \\
1970.3\end{array}$ \\
\hline $\begin{array}{r}64 \\
10 \\
20 \\
30 \\
40 \\
50\end{array}$ & $\begin{array}{l}3580.3 \\
3591.9 \\
3603.5 \\
3615.1 \\
3626.8 \\
3638.5\end{array}$ & $\begin{array}{l}1026.6 \\
1032.8 \\
1039.0 \\
1045.2 \\
1051.4 \\
1057.7\end{array}$ & $74 \begin{array}{r}10 \\
20 \\
30 \\
40 \\
50\end{array}$ & $\begin{array}{l}4317.6 \\
4330.7 \\
4343.8 \\
4356.9 \\
4370.1 \\
4383.3\end{array}$ & $\begin{array}{l}1444.6 \\
1452.5 \\
1460.4 \\
1468.4 \\
1476.4 \\
1484.4\end{array}$ & $84 \begin{array}{r}10 \\
20 \\
30 \\
40 \\
50\end{array}$ & $\begin{array}{l}5159.0 \\
5174.1 \\
5189.3 \\
5204.4 \\
5219.7 \\
5234.9\end{array}$ & $\begin{array}{l}1980.4 \\
1990.5 \\
2000.6 \\
2010.8 \\
2021.1 \\
2031.4\end{array}$ \\
\hline $\begin{array}{r}65 \\
10 \\
20 \\
30 \\
40 \\
50\end{array}$ & $\begin{array}{l}3650.2 \\
3661.9 \\
3673.7 \\
3685.4 \\
3697.2 \\
3709.0\end{array}$ & $\begin{array}{l}1063.9 \\
1070.2 \\
1076.6 \\
1082.9 \\
1089.3 \\
1095.7\end{array}$ & $\begin{array}{r}75 \\
10 \\
20 \\
30 \\
40 \\
50\end{array}$ & $\begin{array}{l}4396.5 \\
4409.8 \\
4423.1 \\
4436.4 \\
4449.7 \\
4463.1\end{array}$ & $\begin{array}{l}1492.4 \\
1500.5 \\
1508.6 \\
1516.7 \\
1524.9 \\
1533.1\end{array}$ & $85 \begin{array}{r}10 \\
10 \\
20 \\
30 \\
40 \\
50\end{array}$ & $\begin{array}{l}5250.3 \\
5265.6 \\
5281.0 \\
5296.4 \\
5311.9 \\
5327.4\end{array}$ & $\begin{array}{l}2041.7 \\
2052.1 \\
2062.5 \\
2073.0 \\
2083.5 \\
2094.1\end{array}$ \\
\hline $\begin{array}{r}66 \\
10 \\
20 \\
30 \\
40 \\
50\end{array}$ & $\begin{array}{l}3720.9 \\
3732.7 \\
3744.6 \\
3756.5 \\
3768.5 \\
3780.4\end{array}$ & $\begin{array}{l}1102.2 \\
1108.6 \\
1115.1 \\
1121.7 \\
1128.2 \\
1134.8\end{array}$ & $\begin{array}{r}76 \\
10 \\
20 \\
30 \\
40 \\
50\end{array}$ & $\begin{array}{l}4476.5 \\
4489.9 \\
4503.4 \\
4516.9 \\
4530.4 \\
4544.0\end{array}$ & $\begin{array}{l}1541.4 \\
1549.7 \\
1558.0 \\
1566.3 \\
1574.7 \\
1583.1\end{array}$ & $86 \begin{array}{r}10 \\
10 \\
20 \\
30 \\
40 \\
50\end{array}$ & $\begin{array}{l}5343.0 \\
5358.6 \\
5374.2 \\
5389.9 \\
5405.6 \\
5421.4\end{array}$ & $\begin{array}{l}2104.7 \\
2115.3 \\
2126.0 \\
2136.7 \\
2147.5 \\
2158.4\end{array}$ \\
\hline $\begin{array}{r}67 \\
10 \\
20 \\
30 \\
40 \\
50\end{array}$ & $\begin{array}{l}3792.4 \\
3804.4 \\
3816.4 \\
3828.4 \\
3840.5 \\
3852.6\end{array}$ & $\begin{array}{l}1141.4 \\
1148.0 \\
1154.7 \\
1161.3 \\
1168.1 \\
1174.8\end{array}$ & $\begin{array}{r}77 \\
10 \\
20 \\
30 \\
40 \\
50\end{array}$ & $\begin{array}{l}4557.6 \\
4571.2 \\
4584.8 \\
4598.5 \\
4612.2 \\
4626.0\end{array}$ & $\begin{array}{l}1591.6 \\
1600.1 \\
1608.6 \\
1617.1 \\
1625.7 \\
1634.4\end{array}$ & $\begin{array}{r}87 \\
10 \\
20 \\
30 \\
40 \\
50\end{array}$ & $\begin{array}{l}5437.2 \\
5453.1 \\
5469.0 \\
5484.9 \\
5500.9 \\
5517.0\end{array}$ & $\begin{array}{l}2169.2 \\
2180.2 \\
2191.1 \\
2202.2 \\
2213.2 \\
2224.3\end{array}$ \\
\hline $68 \begin{array}{r}10 \\
20 \\
30 \\
40 \\
50 \\
\end{array}$ & $\begin{array}{l}3864.7 \\
3876.8 \\
3889.0 \\
3901.2 \\
3913.4 \\
3925.6\end{array}$ & $\begin{array}{l}1181.6 \\
1188.4 \\
1195.2 \\
1202.0 \\
1208.9 \\
1215.8\end{array}$ & $\begin{array}{r}78 \\
10 \\
20 \\
30 \\
40 \\
50\end{array}$ & $\begin{array}{l}4639.8 \\
4653.6 \\
4667.4 \\
4681.3 \\
4695.2 \\
4709.2\end{array}$ & $\begin{array}{l}1643.0 \\
1651.7 \\
1660.5 \\
1669.2 \\
1678.1 \\
1686.9\end{array}$ & $\begin{array}{r}88 \\
10 \\
20 \\
30 \\
40 \\
50\end{array}$ & $\begin{array}{l}5533.1 \\
5549.2 \\
5565.4 \\
5581.6 \\
5597.8 \\
5614.2\end{array}$ & $\begin{array}{l}2235.5 \\
2246.7 \\
2258.0 \\
2269.3 \\
2280.6 \\
2292.0\end{array}$ \\
\hline $\begin{array}{r}69 \\
10 \\
20 \\
30 \\
40 \\
50\end{array}$ & $\begin{array}{l}3937.9 \\
3950.2 \\
3962.5 \\
3974.8 \\
3987.2 \\
3999.5\end{array}$ & $\begin{array}{l}1222.7 \\
1229.7 \\
1236.7 \\
1243.7 \\
1250.8 \\
1257.9\end{array}$ & $\begin{array}{r}79 \\
10 \\
20 \\
30 \\
40 \\
50\end{array}$ & $\begin{array}{l}4723.2 \\
4737.2 \\
4751.2 \\
4765.3 \\
4779.4 \\
4793.6\end{array}$ & $\begin{array}{l}1695.8 \\
1704.7 \\
1713.7 \\
1722.7 \\
1731.7 \\
1740.8\end{array}$ & $\begin{array}{r}89 \\
10 \\
20 \\
30 \\
40 \\
50\end{array}$ & $\begin{array}{l}5630.5 \\
5646.9 \\
5663.4 \\
5679.9 \\
5696.4 \\
5713.0\end{array}$ & $\begin{array}{l}2303.5 \\
2315.0 \\
2326.6 \\
2338.2 \\
2349.8 \\
2361.5\end{array}$ \\
\hline $\begin{array}{r}70 \\
10 \\
20 \\
30 \\
40 \\
50\end{array}$ & $\begin{array}{l}4011.9 \\
4024.4 \\
4036.8 \\
4049.3 \\
4061.8 \\
4074.4\end{array}$ & $\begin{array}{l}1265.0 \\
1272.1 \\
1279.3 \\
1286.5 \\
1293.6 \\
1300.9\end{array}$ & $\begin{array}{r}80 \\
10 \\
20 \\
30 \\
40 \\
50\end{array}$ & $\begin{array}{l}4807.7 \\
4822.0 \\
4836.2 \\
4850.5 \\
4864.8 \\
4879.2\end{array}$ & $\begin{array}{l}1749.9 \\
1759.0 \\
1768.2 \\
1777.4 \\
1786.7 \\
1796.0\end{array}$ & $\begin{array}{r}90 \\
10 \\
20 \\
30 \\
40 \\
50\end{array}$ & $\begin{array}{l}5729.7 \\
5746.3 \\
5763.1 \\
5779.9 \\
5796.7 \\
5813.6\end{array}$ & $\begin{array}{l}2373.3 \\
2385.1 \\
2397.0 \\
2408.9 \\
2420.9 \\
2432.9\end{array}$ \\
\hline
\end{tabular}


Table V.-Tangents and Externals to a $1^{\circ}$ Curve.

\begin{tabular}{|c|c|c|c|c|c|c|c|c|}
\hline $\begin{array}{c}\text { Central } \\
\text { Angle }\end{array}$ & Tangent & External & $\begin{array}{c}\text { Central } \\
\text { Angle }\end{array}$ & Tangent & External & $\begin{array}{c}\text { Central } \\
\text { Angle }\end{array}$ & Tangent & External \\
\hline $\begin{array}{c}91^{\circ} \\
10^{\prime} \\
20 \\
30 \\
40 \\
50\end{array}$ & $\begin{array}{l}5830.5 \\
5847.5 \\
5864.6 \\
5881.7 \\
5898.8 \\
5916.0\end{array}$ & $\begin{array}{l}2444.9 \\
2457.1 \\
2469.3 \\
2481.5 \\
2493.8 \\
2506.1\end{array}$ & $\begin{array}{c}101^{\circ} \\
10^{\prime} \\
20 \\
30 \\
40 \\
50\end{array}$ & $\begin{array}{l}6950.6 \\
6971.3 \\
6992.0 \\
7012.7 \\
7033.6 \\
7054.5\end{array}$ & $\begin{array}{l}3278.1 \\
3294.1 \\
3310.1 \\
3326.1 \\
3342.3 \\
3358.5\end{array}$ & $\begin{array}{c}111^{\circ} \\
10^{\prime} \\
20 \\
30 \\
40 \\
50\end{array}$ & $\begin{array}{l}8336.7 \\
8362.7 \\
8388.9 \\
8415.1 \\
8441.5 \\
8468.0\end{array}$ & $\begin{array}{l}4386.1 \\
4407.6 \\
4429.2 \\
4450.9 \\
4472.7 \\
4494.6\end{array}$ \\
\hline $\begin{array}{l}92 \\
10 \\
20 \\
30 \\
40 \\
50\end{array}$ & $\begin{array}{l}5933.2 \\
5950.5 \\
5967.9 \\
5985.3 \\
6002.7 \\
6020.2\end{array}$ & $\begin{array}{l}2518.5 \\
2531.0 \\
2543.5 \\
2556.0 \\
2568.6 \\
2581.3\end{array}$ & $102 \begin{array}{r}10 \\
20 \\
30 \\
40 \\
50\end{array}$ & $\begin{array}{l}7075.5 \\
7096.6 \\
7117.8 \\
7139.0 \\
7160.3 \\
7181.7\end{array}$ & $\begin{array}{l}3374.9 \\
3391.2 \\
3407.7 \\
3424.3 \\
3440.9 \\
3457.6\end{array}$ & $\begin{array}{r}112 \\
10 \\
20 \\
30 \\
40 \\
50\end{array}$ & $\begin{array}{l}8494.6 \\
8521.3 \\
8548.1 \\
8575.0 \\
8602.1 \\
8629.3\end{array}$ & $\begin{array}{l}4516.6 \\
4538.8 \\
4561.1 \\
4583.4 \\
4606.0 \\
4628.6\end{array}$ \\
\hline $93 \begin{array}{r} \\
10 \\
20 \\
30 \\
40 \\
50\end{array}$ & $\begin{array}{l}6037.8 \\
6055.4 \\
6073.1 \\
6090.8 \\
6108.6 \\
6126.4\end{array}$ & $\begin{array}{l}2594.0 \\
2606.8 \\
2619.7 \\
2632.6 \\
2645.5 \\
2658.5\end{array}$ & 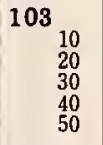 & $\begin{array}{l}7203.2 \\
7224.7 \\
7246.3 \\
7268.0 \\
7289.8 \\
7311.7\end{array}$ & $\begin{array}{l}3474.4 \\
3491.3 \\
3508.2 \\
3525.2 \\
3542.4 \\
3559.6\end{array}$ & $113 \begin{array}{r}10 \\
20 \\
30 \\
40 \\
50\end{array}$ & $\begin{array}{l}8656.6 \\
8684.0 \\
8711.5 \\
8739.2 \\
8767.0 \\
8794.9\end{array}$ & $\begin{array}{l}4651.3 \\
4674.2 \\
4697.2 \\
4720.3 \\
4743.6 \\
4766.9\end{array}$ \\
\hline $94 \begin{array}{r} \\
10 \\
20 \\
30 \\
40 \\
50\end{array}$ & $\begin{array}{l}6144.3 \\
6162.6 \\
6180.2 \\
6198.3 \\
6216.4 \\
6234.6\end{array}$ & $\begin{array}{l}2671.6 \\
2684.7 \\
2697.9 \\
2711.2 \\
2724.5 \\
2737.9\end{array}$ & $\begin{array}{r}104 \\
10 \\
20 \\
30 \\
40 \\
50\end{array}$ & $\begin{array}{l}7333.6 \\
7355.6 \\
7377.8 \\
7399.9 \\
7422.2 \\
7444.6\end{array}$ & $\begin{array}{l}3576.8 \\
3594.2 \\
3611.7 \\
3629.2 \\
3646.8 \\
3664.5\end{array}$ & 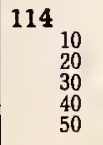 & $\begin{array}{l}8822.9 \\
8851.0 \\
8879.3 \\
8907.7 \\
8936.3 \\
8965.0\end{array}$ & $\begin{array}{l}4790.4 \\
4814.1 \\
4837.8 \\
4861.7 \\
4885.7 \\
4909.9\end{array}$ \\
\hline $95 \begin{array}{r} \\
10 \\
20 \\
30 \\
40 \\
50\end{array}$ & $\begin{array}{l}6252.8 \\
6271.1 \\
6289.4 \\
6307.9 \\
6326.3 \\
6344.8\end{array}$ & $\begin{array}{l}2751.3 \\
2764.8 \\
2778.3 \\
2792.0 \\
2805.6 \\
2819.4\end{array}$ & $\begin{array}{r}105 \\
10 \\
20 \\
30 \\
40 \\
50\end{array}$ & $\begin{array}{l}7467.0 \\
7489.6 \\
7512.2 \\
7534.9 \\
7557.7 \\
7580.5\end{array}$ & $\begin{array}{l}3682.3 \\
3700.2 \\
3718.2 \\
3736.2 \\
3754.4 \\
3772.6\end{array}$ & $\begin{array}{r}115 \\
10 \\
20 \\
30 \\
40 \\
50\end{array}$ & $\begin{array}{l}8993.8 \\
9022.7 \\
9051.7 \\
9080.9 \\
9110.3 \\
9139.8\end{array}$ & $\begin{array}{l}4934.1 \\
4958.6 \\
4983.1 \\
5007.8 \\
5032.6 \\
5057.6\end{array}$ \\
\hline $96 \begin{array}{r}10 \\
20 \\
30 \\
40 \\
50\end{array}$ & $\begin{array}{l}6363.4 \\
6382.1 \\
6400.8 \\
6419.5 \\
6438.4 \\
6457.3\end{array}$ & $\begin{array}{l}2833.2 \\
2847.0 \\
2861.0 \\
2875.0 \\
2889.0 \\
2903.1\end{array}$ & $106 \begin{array}{r}10 \\
20 \\
30 \\
40 \\
50\end{array}$ & $\begin{array}{l}7603.5 \\
7626.6 \\
7649.7 \\
7672.9 \\
7696.3 \\
7719.7\end{array}$ & $\begin{array}{l}3791.0 \\
3809.4 \\
3827.9 \\
3846.5 \\
3865.2 \\
3884.0\end{array}$ & $116 \begin{array}{r}10 \\
20 \\
30 \\
40 \\
50\end{array}$ & $\begin{array}{l}9169.4 \\
9199.1 \\
9229.0 \\
9259.0 \\
9289.2 \\
9319.5\end{array}$ & $\begin{array}{l}5082.7 \\
5107.9 \\
5133.3 \\
5158.8 \\
5184.5 \\
5210.3\end{array}$ \\
\hline $97 \begin{array}{r}10 \\
20 \\
30 \\
40 \\
50\end{array}$ & $\begin{array}{l}6476.2 \\
6495.2 \\
6514.3 \\
6533.4 \\
6552.6 \\
6571.9\end{array}$ & $\begin{array}{l}2917.3 \\
2931.6 \\
2945.9 \\
2960.3 \\
2974.7 \\
2989.2\end{array}$ & $\begin{array}{r}107 \\
10 \\
20 \\
30 \\
40 \\
50\end{array}$ & $\begin{array}{l}7743.2 \\
7766.8 \\
7790.5 \\
7814.3 \\
7838.1 \\
7862.1\end{array}$ & $\begin{array}{l}3902.9 \\
3921.9 \\
3940.9 \\
3960.1 \\
3979.4 \\
3998.7\end{array}$ & $117 \begin{array}{r}10 \\
20 \\
30 \\
40 \\
50\end{array}$ & $\begin{array}{l}9349.9 \\
9380.5 \\
9411.3 \\
9442.2 \\
9473.2 \\
9504.4\end{array}$ & $\begin{array}{l}5236.2 \\
5262.3 \\
5288.6 \\
5315.0 \\
5341.5 \\
5368.2\end{array}$ \\
\hline $98 \begin{array}{r} \\
10 \\
20 \\
30 \\
40 \\
50\end{array}$ & $\begin{array}{l}6591.2 \\
6610.6 \\
6630.1 \\
6649.6 \\
6669.2 \\
6688.8\end{array}$ & $\begin{array}{l}3003.8 \\
3018.4 \\
3033.1 \\
3047.9 \\
3062.8 \\
3077.7\end{array}$ & $\begin{array}{r}108 \\
10 \\
20 \\
30 \\
40 \\
50\end{array}$ & $\begin{array}{l}7886.2 \\
7910.4 \\
7934.6 \\
7959.0 \\
7983.5 \\
8008.0\end{array}$ & $\begin{array}{l}4018.2 \\
4037.8 \\
4057.4 \\
4077.2 \\
4097.1 \\
4117.0\end{array}$ & $\begin{array}{r}118 \\
10 \\
20 \\
30 \\
40 \\
50\end{array}$ & $\begin{array}{l}9535.7 \\
9567.2 \\
9598.9 \\
9630.7 \\
9662.6 \\
9694.7\end{array}$ & $\begin{array}{l}5395.1 \\
5422.1 \\
5449.2 \\
5476.5 \\
5504.0 \\
5531.7\end{array}$ \\
\hline $\begin{array}{l}99 \\
10 \\
20 \\
30 \\
40 \\
50\end{array}$ & $\begin{array}{l}6708.6 \\
6728.4 \\
6748.2 \\
6768.1 \\
6788.1 \\
6808.2\end{array}$ & $\begin{array}{l}3092.7 \\
3107.7 \\
3122.9 \\
3138.1 \\
3153.3 \\
3168.7\end{array}$ & $109 \begin{array}{r}10 \\
20 \\
30 \\
40 \\
50\end{array}$ & $\begin{array}{l}8032.7 \\
8057.4 \\
8082.3 \\
8107.3 \\
8132.3 \\
8157.5\end{array}$ & $\begin{array}{l}4137.1 \\
4157.3 \\
4177.5 \\
4197.9 \\
4218.4 \\
4239.0\end{array}$ & $119 \begin{array}{r}10 \\
20 \\
30 \\
40 \\
50\end{array}$ & $\begin{array}{l}9727.0 \\
9759.4 \\
9792.0 \\
9824.8 \\
9857.7 \\
9890.8\end{array}$ & $\begin{array}{l}\mathbf{5 5 5 9 . 4} \\
5587.4 \\
5615.5 \\
5643.8 \\
5672.3 \\
5700.9\end{array}$ \\
\hline $\begin{array}{r}100 \\
10 \\
20 \\
30 \\
40 \\
50\end{array}$ & $\begin{array}{l}6828.3 \\
6848.5 \\
6868.8 \\
6889.2 \\
6909.6 \\
6930.1\end{array}$ & $\begin{array}{l}3184.1 \\
3199.6 \\
3215.1 \\
3230.8 \\
3246.5 \\
3262.3\end{array}$ & $\begin{array}{r}110 \\
10 \\
20 \\
30 \\
40 \\
50\end{array}$ & $\begin{array}{l}8182.8 \\
8208.2 \\
8233.7 \\
8259.3 \\
8285.0 \\
8310.8\end{array}$ & $\begin{array}{l}4259.7 \\
4280.5 \\
4301.4 \\
4322.4 \\
4343.6 \\
4364.8\end{array}$ & $\begin{array}{r}120 \\
10 \\
20 \\
30 \\
40 \\
50\end{array}$ & $\begin{array}{r}9924.0 \\
9957.5 \\
9991.0 \\
10025.0 \\
10059.0 \\
10093.0\end{array}$ & $\begin{array}{l}5729.7 \\
5758.6 \\
5787.7 \\
5817.0 \\
5846.5 \\
5876.1\end{array}$ \\
\hline
\end{tabular}


Table VI.-Corrections for Tangents and Externals.

These corrections are to be added to the approximate values, found by dividing the tangent, or external, for a $1^{\circ}$ curve (Table IV) by the degree of curve, in order to obtain the true tangents, or externals. Intermediate values may be obtained by interpolation.

FOR TANGENTS ADD

\begin{tabular}{|c|c|c|c|c|c|c|c|c|c|c|c|c|c|c|c|}
\hline \multirow{2}{*}{\multicolumn{2}{|c|}{$\begin{array}{c}\text { Central } \\
\text { Angle }\end{array}$}} & \multicolumn{14}{|c|}{ DEgREE OF CuRVE } \\
\hline & & $5^{\circ}$ & $10^{\circ} 1$ & $15^{\circ} 2$ & $20^{\circ}$ & $25^{\circ}$ & $30^{\circ}$ & $35^{\circ}$ & $40^{\circ}$ & $45^{\circ}$ & $50^{\circ}$ & $55^{\circ}$ & $60^{\circ}$ & $65^{\circ}$ & $70^{\circ}$ \\
\hline \multicolumn{2}{|l|}{$\begin{array}{l}10^{\circ} \\
15^{\circ} \\
20^{\circ} \\
25^{\circ}\end{array}$} & $\begin{array}{l}.03 \\
.04 \\
.06 \\
.08\end{array}$ & \begin{tabular}{l|}
.06 \\
.10 \\
.13 \\
.16
\end{tabular} & $\begin{array}{r}.09 \\
.14 \\
.19 \\
.24\end{array}$ & $\begin{array}{r}.13 \\
.19 \\
+26 \\
.33\end{array}$ & $\begin{array}{l}.16 \\
.24 \\
.32 \\
.40\end{array}$ & $\begin{array}{l}.19 \\
.29 \\
.39 \\
.49\end{array}$ & $\begin{array}{l}.22 \\
.34 \\
.45 \\
.58\end{array}$ & \begin{tabular}{l|l}
2 & .25 \\
4 & .39 \\
5 & .51 \\
8 & .67
\end{tabular} & $\begin{array}{l}.28 \\
.45 \\
.58 \\
.75\end{array}$ & $\begin{array}{l}.31 \\
.51 \\
.65 \\
.83\end{array}$ & $\begin{array}{l}.34 \\
.53 \\
.72 \\
.90\end{array}$ & $\begin{array}{l}.38 \\
.58 \\
.79 \\
.99\end{array}$ & $\begin{array}{r}.42 \\
.63 \\
.84 \\
1.06\end{array}$ & $\begin{array}{r}.46 \\
.68 \\
.90 \\
1.14\end{array}$ \\
\hline \multicolumn{2}{|l|}{$\begin{array}{l}30^{\circ} \\
35^{\circ} \\
40^{\circ} \\
45^{\circ}\end{array}$} & $\begin{array}{l}.10 \\
.11 \\
.13 \\
.15\end{array}$ & $\begin{array}{l}.19 \\
.22 \\
.26 \\
.30\end{array}$ & $\begin{array}{l}.29 \\
.34 \\
.40 \\
.44\end{array}$ & $\begin{array}{l}.39 \\
.47 \\
.53 \\
.60\end{array}$ & $\begin{array}{l}.49 \\
.58 \\
.67 \\
.76\end{array}$ & $\begin{array}{l}.59 \\
.69 \\
.80 \\
.91\end{array}$ & $\begin{array}{r}.69 \\
.70 \\
.93 \\
1.06\end{array}$ & \begin{tabular}{|r|r}
9 & .79 \\
.81 \\
1.06 \\
1.21
\end{tabular} & \begin{tabular}{l|r}
9 & .89 \\
1 & .92 \\
6 & 1.20 \\
1 & 1.37
\end{tabular} & \begin{tabular}{r|r}
9 & .99 \\
2 & 1.04 \\
0 & 1.34 \\
7 & 1.52
\end{tabular} & $\begin{array}{l}1.09 \\
1.29 \\
1.49 \\
1.70\end{array}$ & $\begin{array}{l}1.20 \\
1.42 \\
1.64 \\
1.87\end{array}$ & $\left\{\begin{array}{l}1.29 \\
1.54 \\
1.79 \\
2.04\end{array}\right.$ & $\begin{array}{l}1.39 \\
1.66 \\
1.94 \\
2.21\end{array}$ \\
\hline \multicolumn{2}{|l|}{$\begin{array}{l}50^{\circ} \\
55^{\circ} \\
60^{\circ} \\
65^{\circ}\end{array}$} & $\begin{array}{l}.17 \\
.19 \\
.21 \\
.23\end{array}$ & $\begin{array}{l}.34 \\
.38 \\
.42 \\
.46\end{array}$ & $\begin{array}{l}.51 \\
.57 \\
.63 \\
.69\end{array}$ & \begin{tabular}{l|l}
.68 \\
.76 \\
.84 \\
.93 & 1 \\
.93
\end{tabular} & \begin{tabular}{r|l}
.85 & 1 \\
.95 & 1 \\
1.05 & 1 \\
1.16 & 1
\end{tabular} & $\begin{array}{l}1.02 \\
1.14 \\
1.27 \\
1.40\end{array}$ & $\begin{array}{l}1.19 \\
1.32 \\
1.49 \\
1.64\end{array}$ & $\begin{array}{l}1.36 \\
1.52 \\
1.71 \\
1.88\end{array}$ & \begin{tabular}{l|l}
6 & 1.54 \\
2 & 1.72 \\
& 1.94 \\
& 2.13
\end{tabular} & \begin{tabular}{l|l}
4 & 1.72 \\
2 & 1.92 \\
4 & 2.17 \\
3 & 2.38
\end{tabular} & $\begin{array}{l}1.91 \\
2.14 \\
2.38 \\
2.63\end{array}$ & $\begin{array}{l}2.10 \\
2.35 \\
2.60 \\
2.88\end{array}$ & $\begin{array}{l}2.29 \\
2.56 \\
2.83 \\
3.13\end{array}$ & $\begin{array}{l}2.48 \\
2.77 \\
3.07 \\
3.39\end{array}$ \\
\hline \multicolumn{2}{|l|}{$\begin{array}{l}70^{\circ} \\
75^{\circ} \\
80^{\circ} \\
85^{\circ}\end{array}$} & $\begin{array}{l}.25 \\
.27 \\
.30 \\
.33\end{array}$ & \begin{tabular}{l|l}
.51 & \\
.56 & \\
.61 & \\
.66 & 1
\end{tabular} & $\begin{array}{rl}.76 & 1 . \\
.83 & 1 \\
.91 & 1 . \\
1.00 & 1 .\end{array}$ & $\begin{array}{ll}.02 & 1 \\
.12 & 1 \\
.22 & 1 \\
.33 & 1\end{array}$ & \begin{tabular}{l|l}
1.28 & 1 \\
1.40 & 1 \\
1.53 & 1 \\
1.68 & 2
\end{tabular} & $\begin{array}{l}1.54 \\
1.69 \\
1.84 \\
2.02\end{array}$ & $\begin{array}{l}1.80 \\
1.98 \\
2.15 \\
2.36\end{array}$ & $\begin{array}{l}2.06 \\
2.27 \\
2.46 \\
2.70\end{array}$ & \begin{tabular}{l|l}
6 & 2.33 \\
7 & 2.37 \\
6 & 2.78 \\
0 & 3.05
\end{tabular} & \begin{tabular}{l|l}
3 & 2.60 \\
7 & 2.87 \\
8 & 3.10 \\
5 & 3.40
\end{tabular} & $\begin{array}{l}2.88 \\
3.16 \\
3.44 \\
3.77\end{array}$ & $\begin{array}{l}3.16 \\
3.47 \\
3.78 \\
4.14\end{array}$ & $\begin{array}{l}3.44 \\
3.78 \\
4.12 \\
4.55\end{array}$ & $\begin{array}{l}3.72 \\
4.09 \\
4.46 \\
4.89\end{array}$ \\
\hline \multicolumn{2}{|l|}{$\begin{array}{r}90^{\circ} \\
95^{\circ} \\
100^{\circ} \\
110^{\circ}\end{array}$} & \begin{tabular}{l|l}
.36 & \\
.39 & .43 \\
.51 & 1
\end{tabular} & \begin{tabular}{r|r}
.72 & 1. \\
.79 & 1. \\
.86 & 1. \\
1.03 & 1.
\end{tabular} & $\begin{array}{ll}1.09 & 1 . \\
1.19 & 1 . \\
1.30 & 1 . \\
1.56 & 2 .\end{array}$ & \begin{tabular}{l|l}
.45 & 1 \\
.55 & 2 \\
.74 & 2 \\
08 & 2 \\
.08
\end{tabular} & \begin{tabular}{l|l}
1.83 & 2 \\
2.00 & 2 \\
2.18 & 2 \\
2.61 & 3
\end{tabular} & $\begin{array}{l}2.20 \\
2.40 \\
2.62 \\
3.14\end{array}$ & $\begin{array}{l}2.57 \\
2.80 \\
3.06 \\
3.67\end{array}$ & \begin{tabular}{l|l}
7 & 2.94 \\
0 & 3.20 \\
6 & 3.50 \\
7 & 4.21
\end{tabular} & \begin{tabular}{l|l}
4 & 3.32 \\
0 & 3.61 \\
0 & 3.95 \\
1.95 & 4.76
\end{tabular} & \begin{tabular}{l|l}
2 & 3.70 \\
1 & 4.02 \\
5 & 4.40 \\
6 & 5.31
\end{tabular} & $\begin{array}{l}4.10 \\
4.40 \\
4.88 \\
5.86\end{array}$ & $\begin{array}{l}4.50 \\
4.98 \\
5.37 \\
6.43\end{array}$ & $\left|\begin{array}{l}4.91 \\
5.38 \\
5.85 \\
7.01\end{array}\right|$ & $\begin{array}{l}5.32 \\
5.83 \\
6.34 \\
7.60\end{array}$ \\
\hline \multicolumn{2}{|l|}{$120^{\circ}$} & .62 & \begin{tabular}{l|l}
1.25 & 1.
\end{tabular} & $1.93 \mid 2$. & \begin{tabular}{l|l}
.52 & 3
\end{tabular} & 3.163 & 3.81 & 4.45 & 5.11 & 15.77 & 76.44 & 17.12 & 7.80 & 8.50 & 9.22 \\
\hline \multicolumn{16}{|c|}{ FOR EXTERNALS ADD } \\
\hline \multirow{2}{*}{$\begin{array}{c}\text { Central } \\
\text { Angle }\end{array}$} & \multicolumn{15}{|c|}{ DEGREE OF CURVE } \\
\hline & $5^{\circ}$ & $10^{\circ}$ & $15^{\circ}$ & $20^{\circ}$ & $25^{\circ}$ & $30^{\circ}$ & $0^{\circ}$ & $35^{\circ}$ & $40^{\circ}$ & $45^{\circ}$ & $50^{\circ}$ & $55^{\circ}$ & $60^{\circ}$ & $65^{\circ}$ & $70^{\circ}$ \\
\hline $\begin{array}{l}10^{\circ} \\
15^{\circ} \\
20^{\circ} \\
25^{\circ}\end{array}$ & $\begin{array}{l}.001 \\
.003 \\
.006 \\
.009\end{array}$ & $\begin{array}{l}.003 \\
.007 \\
.011 \\
.018\end{array}$ & $\begin{array}{l}.004 \\
.010 \\
.017 \\
.027\end{array}$ & $\begin{array}{l}.006 \\
.014 \\
.022 \\
.036\end{array}$ & $\begin{array}{l}.00 \\
.01 \\
.02 \\
.04\end{array}$ & $\begin{array}{ll}78 & .00 \\
28 & .02 \\
16 & .05 \\
\end{array}$ & 008 & $\begin{array}{l}009 \\
027 \\
038 \\
065\end{array}$ & $\begin{array}{l}.011 \\
.029 \\
.045 \\
.074\end{array}$ & $\begin{array}{l}.012 \\
.032 \\
.051 \\
.083\end{array}$ & $\begin{array}{l}.014 \\
.035 \\
.057 \\
.093\end{array}$ & $\begin{array}{l}.015 \\
.039 \\
.063 \\
.106\end{array}$ & $\begin{array}{l}.017 \\
.043 \\
.070 \\
.120\end{array}$ & \begin{tabular}{|l|}
.018 \\
.047 \\
.076 \\
.127
\end{tabular} & \begin{tabular}{|l|l}
7 & .020 \\
.051 \\
.083 \\
.135
\end{tabular} \\
\hline $\begin{array}{l}30^{\circ} \\
35^{\circ} \\
40^{\circ} \\
45^{\circ}\end{array}$ & $\begin{array}{l}.013 \\
.018 \\
.023 \\
.030\end{array}$ & $\begin{array}{l}.025 \\
.035 \\
.046 \\
.060\end{array}$ & $\begin{array}{l}.038 \\
.054 \\
.070 \\
.093\end{array}$ & $\begin{array}{l}.051 \\
.072 \\
.093 \\
.119\end{array}$ & $\begin{array}{l}.06 \\
.08 \\
.11 \\
.15\end{array}$ & $\begin{array}{ll}55 & .07 \\
176 & .10 \\
53 & .14 \\
53\end{array}$ & $\begin{array}{l}788 \\
109 \\
141 \\
184\end{array}$ & $\begin{array}{l}.090 \\
.31 \\
.172 \\
216\end{array}$ & $\begin{array}{l}.103 \\
.153 \\
.203 \\
.254\end{array}$ & $\begin{array}{l}.116 \\
.175 \\
.234 \\
.289\end{array}$ & $\begin{array}{l}.129 \\
.197 \\
.265 \\
.325\end{array}$ & $\begin{array}{l}.149 \\
.213 \\
.277 \\
.351\end{array}$ & $\begin{array}{l}.170 \\
.230 \\
.290 \\
-378\end{array}$ & $\begin{array}{l}.179 \\
.247 \\
.315 \\
.411\end{array}$ & $\begin{array}{l}.188 \\
.264 \\
.341 \\
.445\end{array}$ \\
\hline $\begin{array}{l}50^{\circ} \\
55^{\circ} \\
60^{\circ} \\
65^{\circ}\end{array}$ & $\begin{array}{l}.037 \\
.046 \\
.056 \\
.067\end{array}$ & $\begin{array}{l}.075 \\
.093 \\
.112 \\
.135\end{array}$ & $\begin{array}{l}.116 \\
.142 \\
.168 \\
.204\end{array}$ & $\begin{array}{l}.151 \\
.188 \\
.225 \\
.273\end{array}$ & $\begin{array}{l}.18 \\
.23 \\
.28 \\
.34\end{array}$ & $\begin{array}{lll}39 & .22 \\
36 & .28 \\
33 & .34 \\
13 & 41\end{array}$ & $\begin{array}{r}227 \\
340 \\
412\end{array}$ & $\begin{array}{l}266 \\
332 \\
398 \\
483\end{array}$ & $\begin{array}{l}.305 \\
.381 \\
.457 \\
.554\end{array}$ & $\begin{array}{r}.345 \\
.420 \\
.516 \\
.625\end{array}$ & $\begin{array}{l}.384 \\
.479 \\
.575 \\
.697\end{array}$ & $\begin{array}{r}.425 \\
.530 \\
.636 \\
.711\end{array}$ & $\begin{array}{l}.467 \\
.582 \\
.697 \\
.845\end{array}$ & $\begin{array}{l}.508 \\
.641 \\
.774 \\
.922\end{array}$ & $\begin{array}{r}.550 \\
.700 \\
.851 \\
1.01\end{array}$ \\
\hline $\begin{array}{l}70^{\circ} \\
75^{\circ} \\
80^{\circ} \\
85^{\circ}\end{array}$ & $\begin{array}{l}.080 \\
.095 \\
.110 \\
.128\end{array}$ & $\begin{array}{l}.159 \\
.182 \\
.220 \\
.259\end{array}$ & $\begin{array}{l}.240 \\
.286 \\
.332 \\
.391\end{array}$ & $\begin{array}{l}.321 \\
.383 \\
.445 \\
.524\end{array}$ & $\begin{array}{l}.40 \\
.48 \\
.55 \\
.65\end{array}$ & $\begin{array}{ll}33 & .48 \\
50 & .57 \\
77 & .67 \\
7\end{array}$ & $\begin{array}{r}485 \\
578 \\
590 \\
790\end{array}$ & \begin{tabular}{l|l}
568 \\
678 \\
787 \\
926
\end{tabular} & $\begin{array}{r}-652 \\
.777 \\
.903 \\
1.06\end{array}$ & $\begin{array}{r}.735 \\
.877 \\
1.02 \\
1.20\end{array}$ & $\begin{array}{c}.819 \\
.977 \\
1.13 \\
1.34\end{array}$ & $\begin{array}{l}.906 \\
1.07 \\
1.25 \\
1.47\end{array}$ & $\begin{array}{l}.994 \\
1.18 \\
1.38 \\
1.62\end{array}$ & $\begin{array}{l}1.08 \\
1.29 \\
1.50 \\
1.76\end{array}$ & $\begin{array}{l}1.17 \\
1.39 \\
1.62 \\
1.91\end{array}$ \\
\hline $\begin{array}{r}90^{\circ} \\
95^{\circ} \\
10^{\circ} \\
110^{\circ}\end{array}$ & $\begin{array}{l}.149 \\
.174 \\
.200 \\
.268\end{array}$ & \begin{tabular}{|l}
.299 \\
.350 \\
401 \\
.536
\end{tabular} & \begin{tabular}{|l|}
-450 \\
.522 \\
.604 \\
.806 \\
1
\end{tabular} & \begin{tabular}{|c|}
-603 \\
.706 \\
.809 \\
1.08
\end{tabular} & $\begin{array}{r}.75 \\
.98 \\
1.01 \\
1.35\end{array}$ & $\begin{array}{l}66.91 \\
351.06 \\
1.22 \\
1.6\end{array}$ & $\begin{array}{ll}910 & 1 . \\
26 & 1 . \\
23 & 1 . \\
1 .\end{array}$ & \begin{tabular}{l|l}
.07 & 1 \\
.25 & 1 \\
43 & 1 \\
91 & 2
\end{tabular} & $\begin{array}{l}1.22 \\
1.43 \\
1.64 \\
2.20\end{array}$ & $\begin{array}{l}1.38 \\
1.62 \\
1.85 \\
2.48\end{array}$ & $\begin{array}{l}1.54 \\
1.80 \\
2.06 \\
2.76\end{array}$ & $\begin{array}{l}1.70 \\
1.99 \\
2.28 \\
3.05\end{array}$ & $\begin{array}{l}1.87 \\
2.18 \\
2.50 \\
3.35\end{array}$ & $\begin{array}{l}2.03 \\
2.38 \\
2.73 \\
3.66\end{array}$ & $\begin{array}{l}2.20 \\
2.53 \\
2.96 \\
3.96\end{array}$ \\
\hline $120^{\circ}$ & .360 & .721 & 1.08 & 1.45 & 1.82 & 2.1 & & 572 & 2.953 & 3.33 & 3.72 & 4.11 & 4.50 & 4.91 & 5.32 \\
\hline
\end{tabular}




\section{Table VII._Corrections for Sub-Chords}

and Long Chords.

\begin{tabular}{|c|c|c|c|c|c|c|c|c|c|c|c|c|c|c|c|}
\hline \multicolumn{10}{|c|}{ FOR SUB-CHORDS ADD } & \multirow{2}{*}{$\begin{array}{c}\text { Excess } \\
\text { of arc } \\
\text { per } \\
100 \mathrm{ft} .\end{array}$} & \multicolumn{5}{|c|}{ LONG CHORDS } \\
\hline D & 10 & 20 & 30 & 40 & 50 & 60 & 70 & 80 & 90 & & D & 200 & 300 & 400 & 500 \\
\hline $\begin{array}{r}4 \\
6 \\
8 \\
10\end{array}$ & $\begin{array}{l}.00 \\
.00 \\
.01 \\
.01\end{array}$ & $\begin{array}{l}.00 \\
.01 \\
.02 \\
.02\end{array}$ & $\begin{array}{l}.01 \\
.01 \\
.02 \\
.03\end{array}$ & $\begin{array}{l}.01 \\
.02 \\
.03 \\
.04\end{array}$ & $\begin{array}{l}.01 \\
.02 \\
.03 \\
.05\end{array}$ & $\begin{array}{l}.01 \\
.02 \\
.03 \\
.05\end{array}$ & $\begin{array}{l}.01 \\
.02 \\
.03 \\
.05\end{array}$ & $\begin{array}{l}.01 \\
.01 \\
.02 \\
.04\end{array}$ & $\begin{array}{l}.00 \\
.01 \\
.01 \\
.02\end{array}$ & $\begin{array}{l}.02 \\
.05 \\
.08 \\
.13\end{array}$ & $\begin{array}{l}1 \\
\mathbf{2} \\
3 \\
4\end{array}$ & $\begin{array}{l}199.99 \\
199.97 \\
199.93 \\
199.88\end{array}$ & & & $\begin{array}{l}499.85 \\
499.39 \\
498.63 \\
497.57\end{array}$ \\
\hline $\begin{array}{l}12 \\
14 \\
16 \\
18 \\
20\end{array}$ & $\begin{array}{l}.02 \\
.02 \\
.03 \\
.04 \\
.05\end{array}$ & $\begin{array}{l}.04 \\
.05 \\
.06 \\
.08 \\
.10\end{array}$ & $\begin{array}{l}.05 \\
.07 \\
.09 \\
.11 \\
.14\end{array}$ & $\begin{array}{l}.06 \\
.08 \\
.11 \\
.14 \\
.17\end{array}$ & $\begin{array}{l}.07 \\
.09 \\
.12 \\
.15 \\
.19\end{array}$ & $\begin{array}{l}.07 \\
.10 \\
12 \\
.16 \\
.20\end{array}$ & $\begin{array}{l}.07 \\
.09 \\
.12 \\
.15 \\
.18\end{array}$ & $\begin{array}{l}.05 \\
.07 \\
.09 \\
.12 \\
.15\end{array}$ & $\begin{array}{l}.03 \\
.04 \\
.05 \\
.07 \\
.09\end{array}$ & $\begin{array}{l}.18 \\
.25 \\
.33 \\
.41 \\
.51\end{array}$ & $\begin{array}{l}5 \\
6 \\
7 \\
8 \\
\mathbf{9}\end{array}$ & $\begin{array}{l}199.81 \\
199.73 \\
199.63 \\
199.51 \\
199.38\end{array}$ & & & $\begin{array}{l}496.20 \\
494.53 \\
492.57 \\
490.31 \\
487.75\end{array}$ \\
\hline $\begin{array}{l}22 \\
24 \\
26 \\
28 \\
30\end{array}$ & $\begin{array}{l}.06 \\
.07 \\
.09 \\
.10 \\
.11\end{array}$ & $\begin{array}{r}.12 \\
.14 \\
.17 \\
.19 \\
22\end{array}$ & $\begin{array}{l}.17 \\
.20 \\
.24 \\
.27 \\
.31\end{array}$ & $\begin{array}{l}.21 \\
.25 \\
.29 \\
.34 \\
.39\end{array}$ & $\begin{array}{l}.23 \\
.28 \\
.32 \\
.37 \\
.43\end{array}$ & $\begin{array}{l}.24 \\
.28 \\
.33 \\
.38 \\
.44\end{array}$ & $\begin{array}{l}.22 \\
.26 \\
.31 \\
.36 \\
.41\end{array}$ & $\begin{array}{l}.18 \\
.21 \\
.25 \\
.29 \\
.33\end{array}$ & $\begin{array}{l}.10 \\
.12 \\
.15 \\
.17 \\
.19\end{array}$ & $\begin{array}{r}.62 \\
.74 \\
.86 \\
1.00 \\
1.15\end{array}$ & $\begin{array}{l}10 \\
12 \\
14 \\
16 \\
18\end{array}$ & $\begin{array}{l}199.24 \\
198.90 \\
198.51 \\
198.05 \\
197.54\end{array}$ & $\begin{array}{l}296.96 \\
295.63 \\
294.06 \\
292.25 \\
290.21\end{array}$ & $\begin{array}{l}392.42 \\
389.12 \\
385.22 \\
380.76 \\
375.74\end{array}$ & $\begin{array}{l}484.90 \\
478.34 \\
470.65 \\
461.86 \\
452.02\end{array}$ \\
\hline $\begin{array}{l}32 \\
34 \\
36 \\
38 \\
40\end{array}$ & $\begin{array}{l}.13 \\
.15 \\
.17 \\
.18 \\
.21\end{array}$ & $\begin{array}{l}.25 \\
.28 \\
.32 \\
.36 \\
40\end{array}$ & $\begin{array}{l}.36 \\
.40 \\
.45 \\
.51 \\
.56\end{array}$ & $\begin{array}{l}.44 \\
.50 \\
.56 \\
.62 \\
.69\end{array}$ & $\begin{array}{l}.49 \\
.55 \\
.62 \\
.70 \\
.77\end{array}$ & $\begin{array}{l}.50 \\
.57 \\
.64 \\
.71 \\
.79\end{array}$ & $\begin{array}{l}.47 \\
.53 \\
.59 \\
.66 \\
.73\end{array}$ & $\begin{array}{l}.38 \\
.43 \\
.48 \\
.53 \\
.59\end{array}$ & $\begin{array}{l}.22 \\
.25 \\
-28 \\
.31 \\
.35\end{array}$ & $\begin{array}{l}1.31 \\
1.48 \\
1.66 \\
1.86 \\
2.06\end{array}$ & $\begin{array}{l}20 \\
22 \\
24 \\
26 \\
28\end{array}$ & $\begin{array}{l}196.90 \\
196.32 \\
195.63 \\
194.87 \\
194.06\end{array}$ & & $\begin{array}{l}370.17 \\
364.06 \\
357.43 \\
350.30 \\
342.69\end{array}$ & $\begin{array}{l}441.15 \\
429.30 \\
416.53 \\
402.89 \\
388.42\end{array}$ \\
\hline $\begin{array}{l}42 \\
44 \\
46 \\
48 \\
50\end{array}$ & $\begin{array}{l}.23 \\
.25 \\
.27 \\
.30 \\
.32\end{array}$ & $\begin{array}{l}.44 \\
.48 \\
.52 \\
.57 \\
.62\end{array}$ & $\begin{array}{l}.62 \\
.68 \\
.75 \\
.81 \\
.89\end{array}$ & $\begin{array}{r}.76 \\
.84 \\
.92 \\
1.00 \\
1.09\end{array}$ & $\begin{array}{r}.85 \\
.94 \\
1.02 \\
1.12 \\
1.21\end{array}$ & $\begin{array}{r}.87 \\
.96 \\
1.05 \\
1.14 \\
1.24\end{array}$ & $\begin{array}{r}.81 \\
.89 \\
.98 \\
1.06 \\
1.15\end{array}$ & $\begin{array}{l}.65 \\
.72 \\
.78 \\
.86 \\
.93\end{array}$ & $\begin{array}{r}.38 \\
.42 \\
.46 \\
.50 \\
.55\end{array}$ & $\begin{array}{l}2.28 \\
2.50 \\
2.74 \\
2.99 \\
3.24\end{array}$ & $\begin{array}{l}30 \\
32 \\
34 \\
36 \\
38\end{array}$ & $\begin{array}{l}193.18 \\
192.25 \\
191.26 \\
190.21 \\
189.10\end{array}$ & $\begin{array}{l}273.20 \\
269.61 \\
265.81 \\
261.80 \\
257.60\end{array}$ & $\begin{array}{l}334.61 \\
326.08 \\
317.12 \\
307.77 \\
298.03\end{array}$ & $\begin{array}{l}373.20 \\
357.28 \\
340.73 \\
323.61 \\
305.99\end{array}$ \\
\hline $\begin{array}{l}52 \\
54 \\
56 \\
58 \\
60\end{array}$ & $\begin{array}{l}.35 \\
.38 \\
.41 \\
.44 \\
.47\end{array}$ & $\begin{array}{r}.67 \\
.73 \\
.78 \\
.84 \\
.91\end{array}$ & $\begin{array}{l}.96 \\
1.04 \\
1.12 \\
1.20 \\
1.29\end{array}$ & $\begin{array}{l}1.18 \\
1.28 \\
1.38 \\
1.48 \\
1.59\end{array}$ & $\begin{array}{l}1.31 \\
1.42 \\
1.53 \\
1.65 \\
1.76\end{array}$ & $\begin{array}{l}1.35 \\
1.46 \\
1.67 \\
1.81\end{array}$ & $\begin{array}{l}1.25 \\
1.35 \\
1.46 \\
1.57 \\
1.68\end{array}$ & $\begin{array}{l}1.01 \\
1.09 \\
1.17 \\
1.20 \\
1.35\end{array}$ & $\begin{array}{l}.59 \\
.64 \\
.69 \\
.74 \\
.80\end{array}$ & $\begin{array}{l}3.52 \\
3.80 \\
4.09 \\
4.40 \\
4.72\end{array}$ & $\begin{array}{l}40 \\
42 \\
44 \\
46 \\
48\end{array}$ & $\begin{array}{l}187.94 \\
186.72 \\
185.44 \\
184.10 \\
182.71\end{array}$ & $\begin{array}{l}253.21 \\
248.63 \\
243.87 \\
239.93 \\
233.83\end{array}$ & \begin{tabular}{|}
287.94 \\
277.51 \\
266.78 \\
255.78 \\
244.51
\end{tabular} & $\begin{array}{l}287.94 \\
269.54 \\
250.85 \\
231.95 \\
212.92\end{array}$ \\
\hline & & & & & & & & & & & & & & & \\
\hline
\end{tabular}

Table VIII._Middle Ordinates for Rails _n Feet.

\begin{tabular}{|c|c|c|c|c|c|c|c|c|c|c|c|c|c|c|c|}
\hline \multirow{2}{*}{$\begin{array}{c}\text { Deg. } \\
\text { of } \\
\text { Curve }\end{array}$} & \multicolumn{7}{|c|}{ LENGTH OF RAILS } & \multirow{2}{*}{$\begin{array}{c}\text { Deg. } \\
\text { of } \\
\text { Curve }\end{array}$} & \multicolumn{7}{|c|}{ LENGTH OF RA1LS } \\
\hline & 32 & 30 & 28 & 26 & 24 & 22 & 20 & & 32 & 30 & 28 & 26 & 24 & 22 & 20 \\
\hline $\begin{array}{r}1^{\circ} \\
2 \\
3 \\
4 \\
5 \\
6 \\
7 \\
8 \\
9 \\
10 \\
11 \\
12 \\
13 \\
14 \\
15\end{array}$ & $\begin{array}{l}.022 \\
.045 \\
.067 \\
.089 \\
.112 \\
.134 \\
.156 \\
.179 \\
.201 \\
.223 \\
.245 \\
.268 \\
.290 \\
.312 \\
.334\end{array}$ & $\begin{array}{l}.020 \\
.038 \\
.058 \\
.079 \\
.099 \\
.117 \\
.137 \\
.158 \\
.175 \\
.196 \\
.216 \\
.236 \\
.254 \\
.275 \\
.295\end{array}$ & $\begin{array}{l}.016 \\
.034 \\
.051 \\
.069 \\
.086 \\
.102 \\
.120 \\
.137 \\
.153 \\
.171 \\
.188 \\
.206 \\
.222 \\
.239 \\
.257\end{array}$ & $\begin{array}{l}.013 \\
.029 \\
.044 \\
.060 \\
.074 \\
.088 \\
.104 \\
.119 \\
.133 \\
.148 \\
.163 \\
.179 \\
.192 \\
.207 \\
.223\end{array}$ & $\begin{array}{l}.011 \\
.025 \\
.037 \\
.050 \\
.063 \\
.076 \\
.088 \\
.100 \\
.112 \\
125 \\
.139 \\
.151 \\
.163 \\
.175 \\
.188\end{array}$ & $\begin{array}{l}.009 \\
.021 \\
.031 \\
.042 \\
.053 \\
.064 \\
.074 \\
.085 \\
.095 \\
.106 \\
.117 \\
.128 \\
.138 \\
.148 \\
.159\end{array}$ & $\begin{array}{l}.008 \\
.017 \\
.026 \\
.035 \\
.044 \\
.052 \\
.061 \\
.070 \\
.078 \\
.087 \\
.096 \\
.105 \\
.113 \\
.122 \\
.131\end{array}$ & $\begin{array}{l}16^{\circ} \\
17 \\
18 \\
19 \\
20 \\
21 \\
22 \\
23 \\
24 \\
25 \\
26 \\
27 \\
28 \\
29 \\
30\end{array}$ & $\begin{array}{l}.356 \\
.378 \\
.400 \\
.423 \\
.445 \\
.466 \\
.487 \\
.509 \\
.531 \\
.552 \\
.573 \\
.594 \\
.618 \\
.638 \\
.660\end{array}$ & $\begin{array}{l}.313 \\
.333 \\
.351 \\
.371 \\
.392 \\
.410 \\
.430 \\
.450 \\
.469 \\
.486 \\
.506 \\
.524 \\
.545 \\
.564 \\
.583\end{array}$ & $\begin{array}{l}.273 \\
.290 \\
.306 \\
.324 \\
.341 \\
.357 \\
.375 \\
.390 \\
.408 \\
.424 \\
.441 \\
.457 \\
.475 \\
.491 \\
.508\end{array}$ & $\begin{array}{l}.236 \\
.252 \\
.265 \\
.280 \\
.296 \\
.309 \\
.325 \\
.338 \\
.354 \\
.367 \\
.382 \\
.396 \\
.411 \\
.424 \\
.438\end{array}$ & $\begin{array}{l}.200 \\
.213 \\
.225 \\
.238 \\
.250 \\
.262 \\
.275 \\
.287 \\
.299 \\
.311 \\
.323 \\
.335 \\
.348 \\
.361 \\
.374\end{array}$ & $\begin{array}{l}.170 \\
.180 \\
.190 \\
.201 \\
.212 \\
.222 \\
.233 \\
.243 \\
.253 \\
.263 \\
.274 \\
.284 \\
.303 \\
.313\end{array}$ & $\begin{array}{r}.139 \\
.148 \\
.156 \\
.165 \\
.174 \\
.182 \\
.191 \\
.199 \\
.208 \\
.216 \\
.225 \\
.233 \\
.242 \\
.250 \\
.259\end{array}$ \\
\hline
\end{tabular}


TRIGONOMETRIC FORMULE

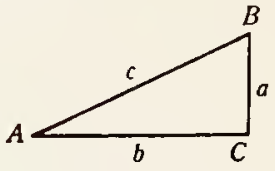

Right Triangle

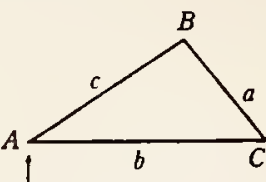

Oblique Triangles

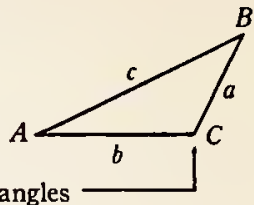

\section{Solution of Right Triangles}

For Angle $A \cdot \sin =\frac{a}{c}, \cos =\frac{b}{c}, \tan =\frac{a}{b}, \cot =\frac{b}{a}, \sec =\frac{c}{b}, \operatorname{cosec}=\frac{c}{a}$

Given

$a, c$

$A, a$

$A, b$

$A, c$

Given

$A, B, a$

$A, a, b$

$a, b, c$

$A, B, c$

$a, b, c$

$A, B, C$

$\begin{aligned} & \text { Required } \\ & A, B, c \\ & A, B, b\end{aligned}$

$B, b, c$

$B, a, c$

$B, a, b$

$B, c, C$

$\tan A=\frac{a}{b}=\cot B, c=\sqrt{a^{2}+b^{2}}=a \sqrt{1+\frac{b^{2}}{a^{2}}}$

$\sin A=\frac{a}{c}=\cos B, b=\sqrt{(c+a)(c-a)}=c \sqrt{1-\frac{a^{2}}{c^{2}}}$

$B=90^{\circ}-A, b=a \cot A, c=\frac{a}{\sin A}$.

$B=90^{\circ}-A, a=b \tan A, c=\frac{b}{\cos A}$.

$B=90^{\circ}-A, a=c \sin A, b=c \cos A$.

\section{Solution of Oblique Triangles}

$b, c, C \quad b=\frac{a \sin B}{\sin A}, C=180^{\circ}-(A+B), c=\frac{a \sin C}{\sin A}$

$\sin B=\frac{b \sin A}{a}, C=180^{\circ}-(A+B), c=\frac{a \sin C}{\sin A}$

$A+B=180^{\circ}-C, \tan \frac{1}{2}(A-B)=\frac{(a-b) \tan \frac{1}{2}(A+B)}{a+b}$

$$
c=\frac{a \sin C}{\sin A}
$$

$s=\frac{a+b+c}{2}, \sin \frac{1}{2} A=\sqrt{\frac{(s-b)(s-c)}{b c}}$

$$
\sin \frac{1}{2} B=\sqrt{\frac{(s-a)(s-c)}{a c}}, C=180^{\circ}-(A+B)
$$

$a, b, c$

Area

$s=\frac{a+b+c}{2}$, area $=\sqrt{s(s-a)(s-b)(s-c)}$

$A, b, c$

Area

area $=\frac{b c \sin A}{2}$

$A, B, C, a$

area $=\frac{a^{2} \sin B \sin C}{2 \sin A}$

\section{REDUCTION TO HORIZONTAL}

Horizontal distance $=$ slope distance multiplied by the cosine of the vertical angle. Thus, for a slope distance of $403.6 \mathrm{ft}$. and a vertical angle of $4^{\circ} 40^{\prime}$ - the cosine of $4^{\circ} 40^{\circ}$, taker from a table of natural trigonometrical functions, $=.9967$, and honzontal distance $=403.6 \times .9967$ $-402.27 \mathrm{re}$

Horizontal distance also $=5 l o p e$ distance minus slope dis*

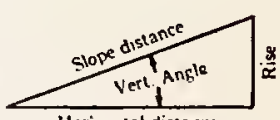

Horizontal distance

tance times $(1-$ cosine of vertucal angle), Using the same figures as in the preceding example $\operatorname{Cos} .4^{*} 40^{2}=.9967 .1-9967=.0033 .403 .6 \times .0033=1.33$ ft. Horizonts] dist, $=403.6-1.33=402.27$ [t

When the nse is known. the horizontal distance may be found by the following approximate rule:- the slope distance less the square of the rise divided by twice the slope distance. Thus. for a slope distance of $372.5 \mathrm{ft}$., and a nse of $15 \mathrm{ft}$. the horizonlal distance $=$

$$
3725-\frac{15 \times 15}{2 \times 372.5}=372.5-.30=372.2 \mathrm{ft} \text {. }
$$


Table IX.-Natural Trigonometrical Functions.

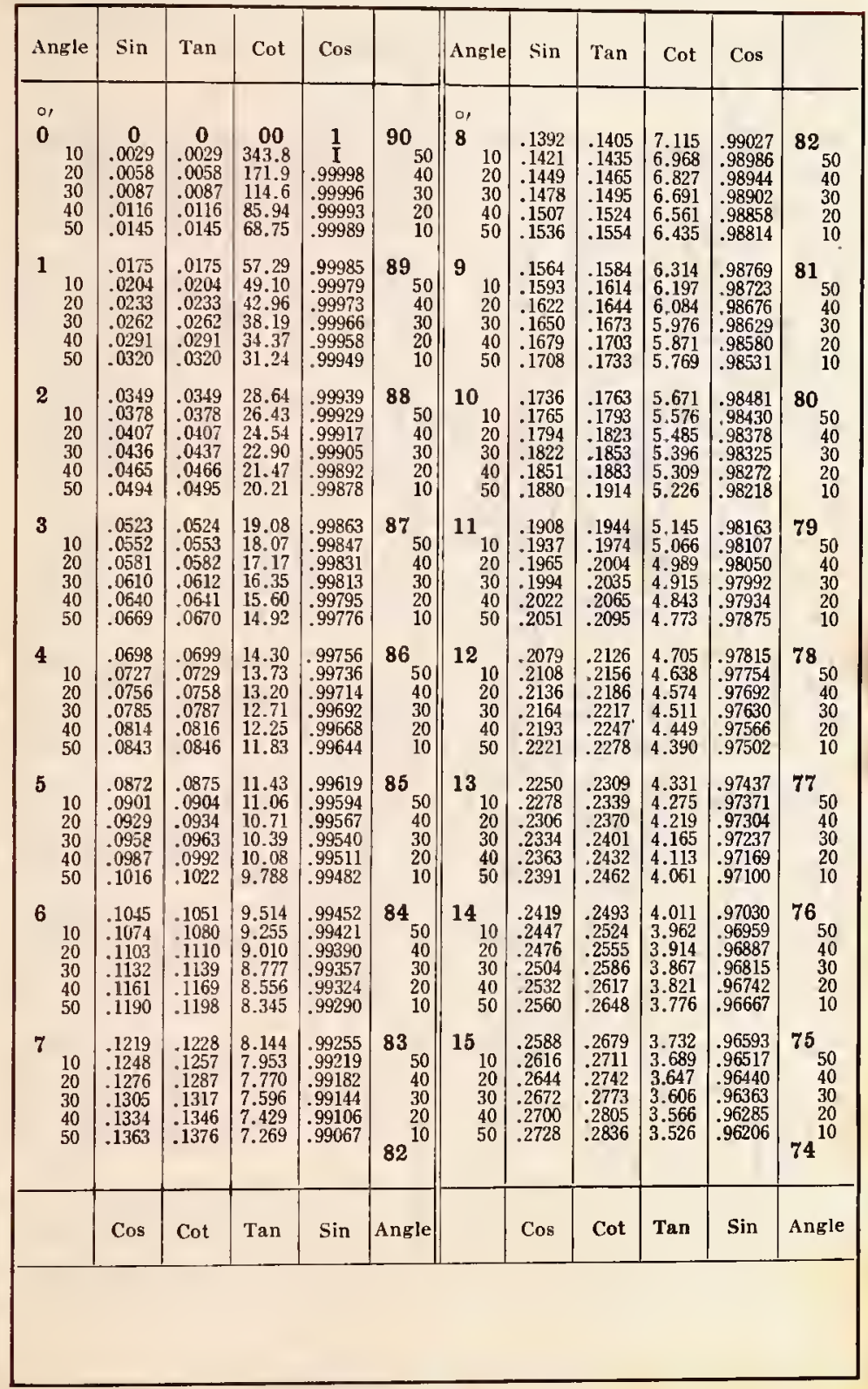


Table IX.-Natural Trigonometrical Functions.

\begin{tabular}{|c|c|c|c|c|c|c|c|c|c|c|c|}
\hline Angle & $\sin$ & Tan & Cot & $\operatorname{Cos}$ & & Angle & $\operatorname{Sin}$ & Tan. & Cot & $\operatorname{Cos}$ & \\
\hline $\begin{array}{l}16 \\
16 \\
10 \\
20 \\
30 \\
40 \\
50\end{array}$ & $\begin{array}{l}.2756 \\
.2784 \\
.2812 \\
.2840 \\
.2868 \\
.2896\end{array}$ & $\begin{array}{l}.2867 \\
.2899 \\
.2931 \\
.2962 \\
.2994 \\
.3026\end{array}$ & $\begin{array}{l}3.487 \\
3.450 \\
3.412 \\
3.376 \\
3.340 \\
3.305\end{array}$ & $\begin{array}{l}.96126 \\
.96046 \\
.95964 \\
.95882 \\
.95799 \\
.95715\end{array}$ & $\begin{array}{r}74 \\
50 \\
40 \\
30 \\
20 \\
10\end{array}$ & $\begin{array}{r}24 \\
24 \\
10 \\
20 \\
30 \\
40 \\
50\end{array}$ & $\begin{array}{l}.4067 \\
.4094 \\
.4120 \\
.4147 \\
.4173 \\
.4200\end{array}$ & $\begin{array}{l}.4452 \\
.4487 \\
.4522 \\
.4557 \\
.4592 \\
.4628\end{array}$ & $\begin{array}{l}2.246 \\
2.229 \\
2.211 \\
2.194 \\
2.177 \\
2.161\end{array}$ & $\begin{array}{l}.91355 \\
.91236 \\
.91116 \\
.90996 \\
.90875 \\
.90753\end{array}$ & $\begin{array}{r}66 \\
50 \\
40 \\
30 \\
20 \\
10\end{array}$ \\
\hline $\begin{array}{r}17 . \\
10 \\
20 \\
30 \\
40 \\
50\end{array}$ & $\begin{array}{l}.2924 \\
.2952 \\
.2979 \\
.3007 \\
.3035 \\
.3062\end{array}$ & $\begin{array}{r}.3057 \\
.3089 \\
.3121 \\
.3153 \\
.3185 \\
+3217\end{array}$ & $\begin{array}{l}3.271 \\
3.237 \\
3.204 \\
3.172 \\
3.140 \\
3.108\end{array}$ & $\begin{array}{r}.95630 \\
.95545 \\
.95459 \\
.95372 \\
.95284 \\
.95195\end{array}$ & $\begin{array}{r}73 \\
50 \\
40 \\
30 \\
20 \\
10\end{array}$ & $\begin{array}{r}25 \\
10 \\
20 \\
30 \\
40 \\
50\end{array}$ & $\begin{array}{l}.4226 \\
.4253 \\
.4279 \\
.4305 \\
.4331 \\
.4358\end{array}$ & $\begin{array}{l}.4663 \\
.4699 \\
.4734 \\
.4770 \\
.4806 \\
.4841\end{array}$ & $\begin{array}{l}2.145 \\
2.128 \\
2.112 \\
2.097 \\
2.081 \\
2.066\end{array}$ & $\begin{array}{l}.90631 \\
.90507 \\
.90383 \\
.90259 \\
.90133 \\
.90007\end{array}$ & $\begin{array}{r}65 \\
50 \\
40 \\
30 \\
20 \\
10\end{array}$ \\
\hline $\begin{array}{r}18 \\
10 \\
20 \\
30 \\
40 \\
50\end{array}$ & $\begin{array}{l}.3090 \\
.3118 \\
.3145 \\
.3173 \\
.3201 \\
.3228\end{array}$ & $\begin{array}{l}.3249 \\
.3281 \\
.3314 \\
.3346 \\
.3378 \\
.3411\end{array}$ & $\begin{array}{l}3.078 \\
3.048 \\
3.018 \\
2.989 \\
2.960 \\
2.932\end{array}$ & $\begin{array}{l}.95106 \\
.95015 \\
.94924 \\
.94832 \\
.94740 \\
.94646\end{array}$ & \begin{tabular}{r|}
72 \\
50 \\
40 \\
30 \\
20 \\
10
\end{tabular} & $\begin{array}{r}26 \\
10 \\
20 \\
30 \\
40 \\
50\end{array}$ & $\begin{array}{l}.4384 \\
.4410 \\
.4436 \\
.4462 \\
.4488 \\
.4514\end{array}$ & $\begin{array}{l}.4877 \\
.4913 \\
.4950 \\
.4986 \\
.5022 \\
.5059\end{array}$ & $\begin{array}{l}2.050 \\
2.035 \\
2.020 \\
2.006 \\
1.991 \\
1.977\end{array}$ & $\begin{array}{l}.89879 \\
.89752 \\
.89623 \\
.89493 \\
.89363 \\
.89232\end{array}$ & $\begin{array}{r}64 \\
50 \\
40 \\
30 \\
20 \\
10\end{array}$ \\
\hline $\begin{array}{r}19 \\
10 \\
20 \\
30 \\
40 \\
50\end{array}$ & $\begin{array}{l}.3256 \\
.3283 \\
.3311 \\
.3338 \\
.3365 \\
.3393\end{array}$ & $\begin{array}{l}.3443 \\
.3476 \\
.3508 \\
.3541 \\
.3574 \\
.3607\end{array}$ & $\begin{array}{l}2.904 \\
2.877 \\
2.850 \\
2.824 \\
2.798 \\
2.773\end{array}$ & $\begin{array}{r}.94552 \\
.94457 \\
.94361 \\
.94264 \\
.94167 \\
.94068\end{array}$ & $\begin{array}{r}71 \\
50 \\
40 \\
30 \\
20 \\
10\end{array}$ & $\begin{array}{r}27 \\
10 \\
20 \\
30 \\
40 \\
50\end{array}$ & $\begin{array}{l}.4540 \\
.4566 \\
.4592 \\
.4617 \\
.4643 \\
.4669\end{array}$ & $\begin{array}{l}.5095 \\
.5132 \\
.5169 \\
.5206 \\
.5243 \\
.5280\end{array}$ & $\begin{array}{l}1.963 \\
1.949 \\
1.935 \\
1.921 \\
1.907 \\
1.894\end{array}$ & $\begin{array}{l}.89101 \\
.88968 \\
.88835 \\
.88701 \\
.88565 \\
.88431\end{array}$ & $\begin{array}{r}63 \\
50 \\
40 \\
30 \\
20 \\
10\end{array}$ \\
\hline $\begin{array}{r}20 \\
10 \\
20 \\
30 \\
40 \\
50\end{array}$ & $\begin{array}{l}.3420 \\
.3448 \\
.3475 \\
.3502 \\
.3529 \\
.3557\end{array}$ & $\begin{array}{l}.3640 \\
.3673 \\
.3706 \\
.3739 \\
.3772 \\
.3805\end{array}$ & $\begin{array}{l}2.747 \\
2.723 \\
2.699 \\
2.675 \\
2.651 \\
2.628\end{array}$ & $\begin{array}{l}.93969 \\
.93869 \\
.93769 \\
.93667 \\
.93565 \\
.93462\end{array}$ & \begin{tabular}{r|}
70 \\
50 \\
40 \\
30 \\
20 \\
10
\end{tabular} & $\begin{array}{r}28 \\
10 \\
20 \\
30 \\
40 \\
50\end{array}$ & $\begin{array}{l}.4695 \\
.4720 \\
.4746 \\
.4772 \\
.4797 \\
.4823\end{array}$ & $\begin{array}{l}.5317 \\
.5354 \\
.5392 \\
.5430 \\
.5467 \\
.5505\end{array}$ & $\begin{array}{l}1.881 \\
1.868 \\
1.855 \\
1.842 \\
1.829 \\
1.816\end{array}$ & $\begin{array}{l}.88295 \\
.88158 \\
.88020 \\
.87882 \\
.87743 \\
.87603\end{array}$ & $\begin{array}{r}62 \\
50 \\
40 \\
30 \\
20 \\
10\end{array}$ \\
\hline $\begin{array}{r}21 \\
10 \\
20 \\
30 \\
40 \\
50\end{array}$ & $\begin{array}{l}.3584 \\
.3611 \\
.3638 \\
.3665 \\
.3692 \\
.3719\end{array}$ & $\begin{array}{l}.3839 \\
.3872 \\
.3906 \\
.3939 \\
.3973 \\
.4006\end{array}$ & $\begin{array}{l}2.605 \\
2.583 \\
2.560 \\
2.539 \\
2.517 \\
2.496\end{array}$ & $\begin{array}{l}.93358 \\
.93253 \\
.93148 \\
.93042 \\
.92935 \\
.92827\end{array}$ & \begin{tabular}{r||}
69 \\
50 \\
40 \\
30 \\
20 \\
10
\end{tabular} & $\begin{array}{r}29 \\
10 \\
20 \\
30 \\
40 \\
50\end{array}$ & $\begin{array}{l}.4848 \\
.4874 \\
.4899 \\
.4924 \\
.4950 \\
.4975\end{array}$ & $\begin{array}{l}5543 \\
.5581 \\
.5619 \\
.5658 \\
.5696 \\
.5735\end{array}$ & $\begin{array}{l}1.804 \\
1.792 \\
1.780 \\
1.767 \\
1.756 \\
1.744\end{array}$ & $\begin{array}{l}.87462 \\
.87321 \\
.87178 \\
.87036 \\
.86892 \\
.86748\end{array}$ & $\begin{array}{r}61 \\
50 \\
40 \\
30 \\
20 \\
10\end{array}$ \\
\hline $\begin{array}{r}22 \\
10 \\
20 \\
30 \\
40 \\
50\end{array}$ & $\begin{array}{l}.3746 \\
.3773 \\
.3800 \\
.3827 \\
.3854 \\
.3881\end{array}$ & $\begin{array}{l}.4040 \\
.4074 \\
.4108 \\
.4142 \\
.4176 \\
.4210\end{array}$ & $\begin{array}{l}2.475 \\
2.455 \\
2.434 \\
2.414 \\
2.394 \\
2.375\end{array}$ & $\begin{array}{r}.92718 \\
.92609 \\
.92499 \\
.92388 \\
.92276 \\
.92164\end{array}$ & $\begin{array}{r}68 \\
50 \\
40 \\
30 \\
20 \\
10\end{array}$ & $\begin{array}{r}30 \\
10 \\
20 \\
30 \\
40 \\
50\end{array}$ & $\begin{array}{l}.5000 \\
.5025 \\
.5050 \\
.5075 \\
.5100 \\
.5125\end{array}$ & $\begin{array}{l}.5774 \\
.5812 \\
.5851 \\
.5890 \\
.5930 \\
.5969\end{array}$ & $\begin{array}{l}1.732 \\
1.720 \\
1.709 \\
1.698 \\
1.686 \\
1.675\end{array}$ & $\begin{array}{l}.86603 \\
.86457 \\
.86310 \\
.86163 \\
.86015 \\
.85866\end{array}$ & $\begin{array}{r}60 \\
50 \\
40 \\
30 \\
20 \\
10\end{array}$ \\
\hline $\begin{array}{r}23 \\
10 \\
20 \\
30 \\
40 \\
50\end{array}$ & $\begin{array}{l}.3907 \\
.3934 \\
.3961 \\
.3987 \\
.4014 \\
.4041\end{array}$ & $\begin{array}{l}.4245 \\
.4279 \\
.4314 \\
.4348 \\
.4383 \\
.4417\end{array}$ & $\begin{array}{l}2.356 \\
2.337 \\
2.318 \\
2.300 \\
2.282 \\
2.264\end{array}$ & $\begin{array}{l}92050 \\
.91936 \\
.91822 \\
.91706 \\
.91590 \\
.91472\end{array}$ & $\begin{array}{r}67 \\
50 \\
40 \\
30 \\
20 \\
10 \\
66\end{array}$ & $\begin{array}{r}31 \\
10 \\
20 \\
30 \\
40 \\
50\end{array}$ & $\begin{array}{l}.5150 \\
.5175 \\
.5200 \\
.5225 \\
.5250 \\
.5275\end{array}$ & $\begin{array}{l}.6009 \\
.6048 \\
.6088 \\
.6128 \\
.6168 \\
.6208\end{array}$ & $\begin{array}{l}1.664 \\
1.653 \\
1.643 \\
1.632 \\
1.621 \\
1.611\end{array}$ & $\begin{array}{l}.85717 \\
.85567 \\
.85416 \\
.85264 \\
.85112 \\
.84959\end{array}$ & $\begin{array}{r}59 \\
50 \\
40 \\
30 \\
20 \\
10 \\
58\end{array}$ \\
\hline & Cos & Cot & Tan. & $\operatorname{Sin}$ & Angle & & Cos & Cot & Tan. & Sin & Angle \\
\hline
\end{tabular}




\section{Table IX.-Natural Trigonometrical Functions.}

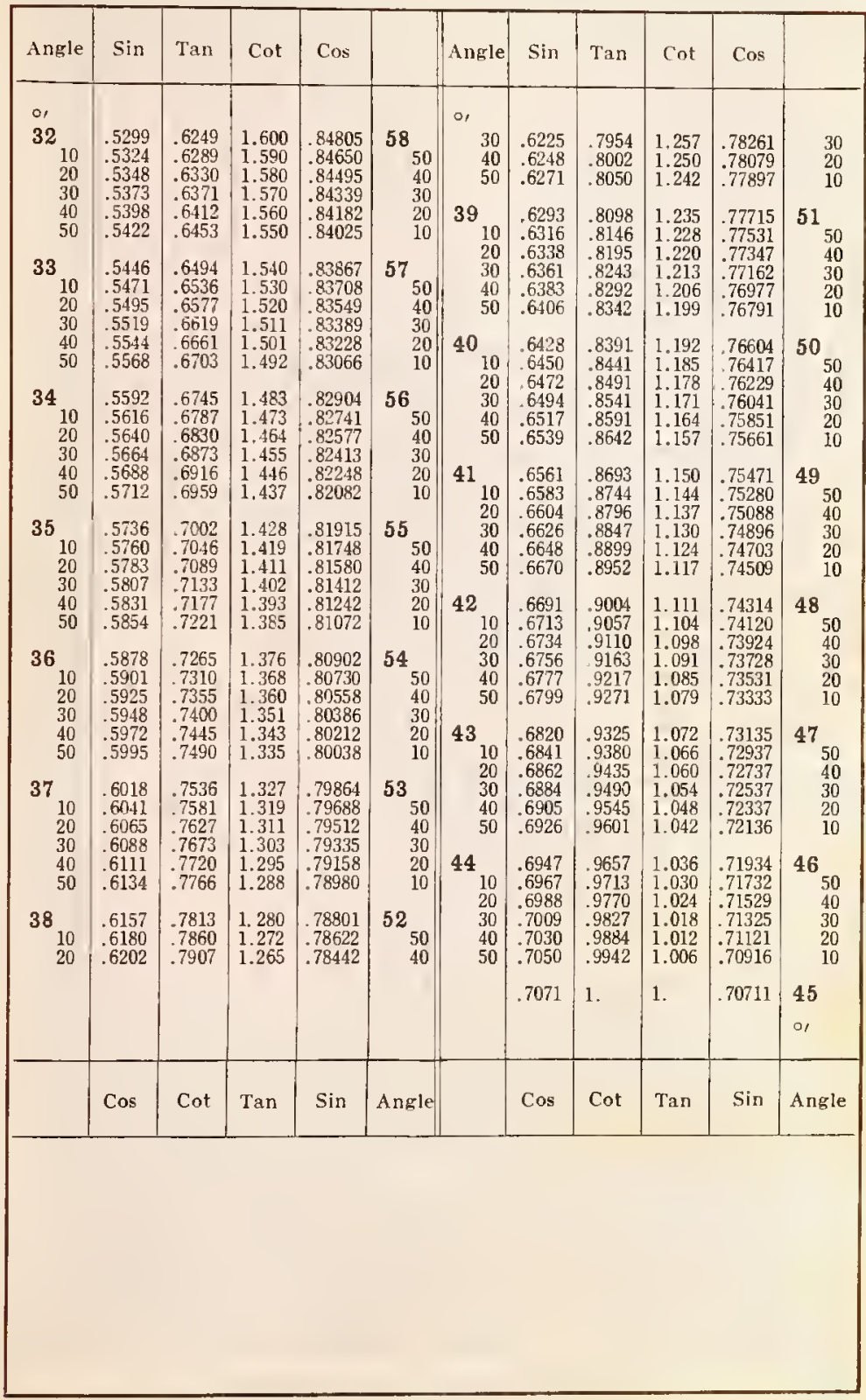




\section{STADIA REDUCTION DIAGRAM}

Enter on the horizontal scale with the value of the atadia rearling (the interval times the stedia constant) and run vertically upward to intersection with liae representing the vertical angle. The location of this point with reference to the dotted lines marked "ONE" etc. gives the correction to be subtracted from the entering value, and to which "i $+c^{\prime \prime}$ (usually about 1 foot) must be added to obtain the true horizontal distance. The reading on the vertical scale plus about $0.1 \mathrm{ft}$. for each $5^{\circ}$ of vertical angle $[(f+c) \sin \alpha]$ is the true vertical distance.

If the vertical angle is beyond the range of diagram, use $H=R \cos ^{2} \alpha+1$ to find true horizontal distance and $V=R \times 1 / 2 \sin 2 \alpha$ for true vertical d istane e wh t r e $R$

equals obs e r y e d s t a d i a d istance and $\alpha$ equals vertical angle.
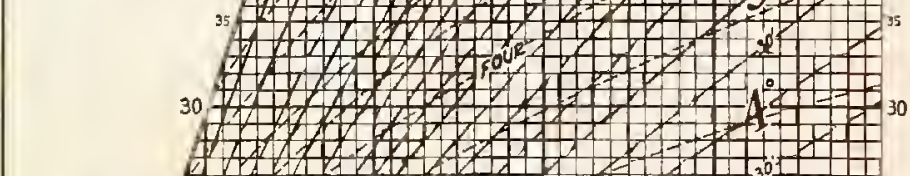
4726
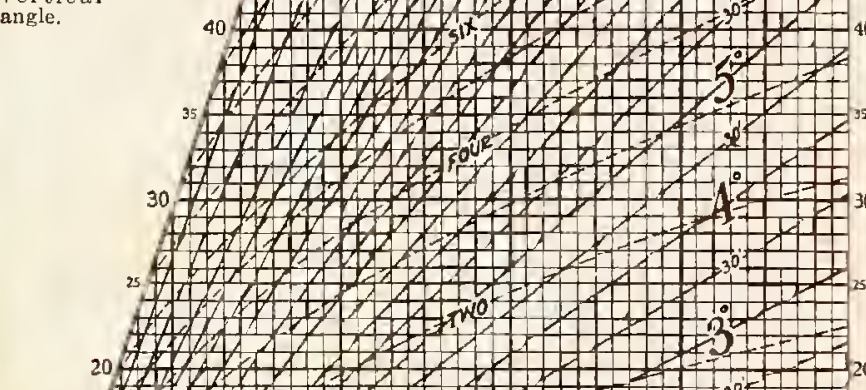


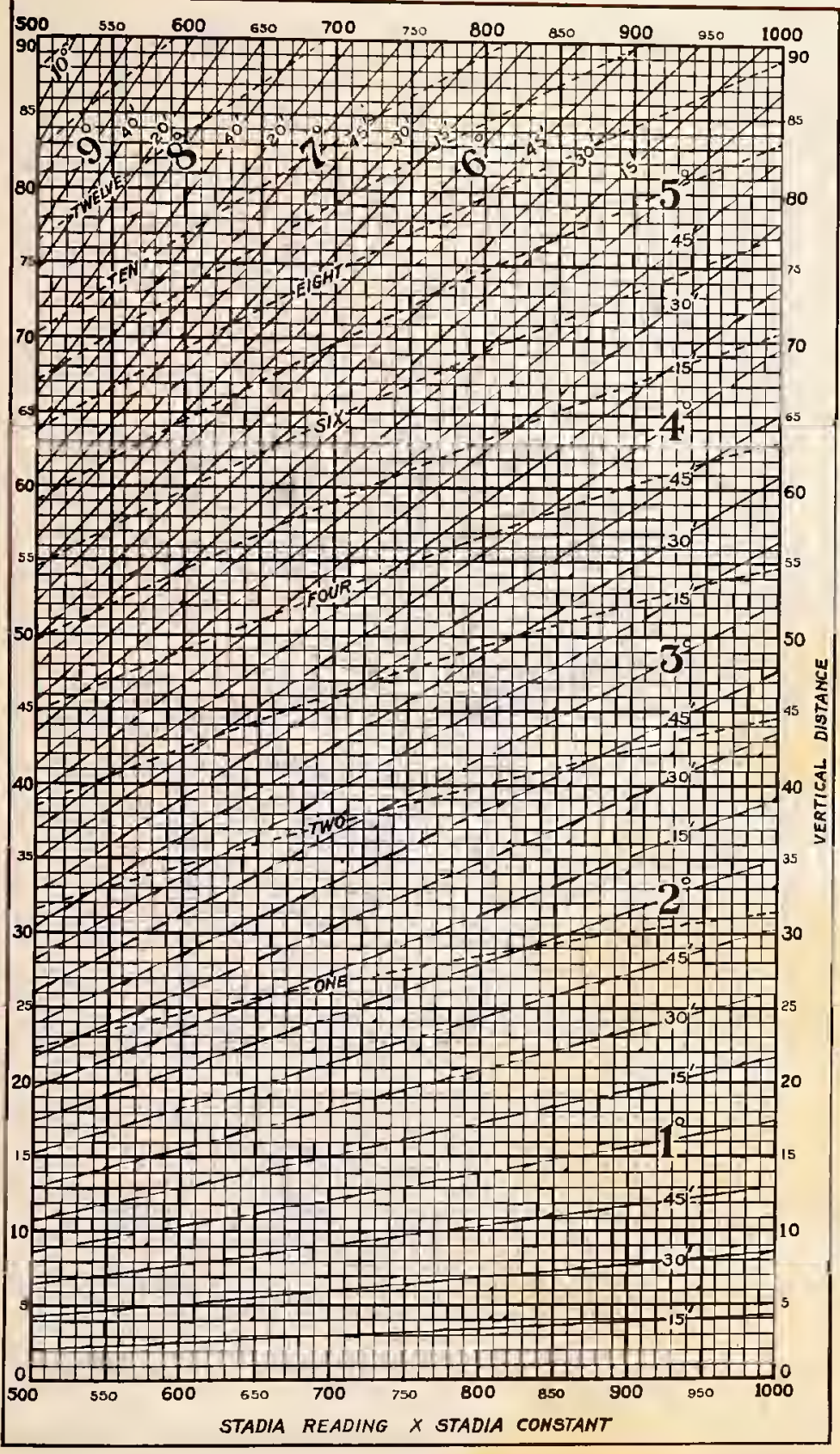




\section{Table X.-Calculation of Earthwork.}

\begin{tabular}{|c|c|c|c|c|c|c|c|c|c|c|c|c|c|c|c|}
\hline \multirow{2}{*}{$\frac{5}{0}$} & \multicolumn{15}{|c|}{ HEIGH' } \\
\hline & 1 & 2 & 3 & 4 & 5 & 6 & 7 & 8 & 9 & 10 & 11 & 12 & 13 & 14 & 15 \\
\hline $\begin{array}{l}\mathbf{1} \\
\mathbf{2} \\
3 \\
\mathbf{4} \\
5 \\
5\end{array}$ & $\begin{array}{l}.02 \\
.04 \\
.06 \\
.07 \\
.09\end{array}$ & $\begin{array}{l}.04 \\
.07 \\
.11 \\
.15 \\
.19\end{array}$ & $\begin{array}{l}.06 \\
.11 \\
.17 \\
.22 \\
.28\end{array}$ & $\begin{array}{l}.07 \\
.15 \\
.22 \\
.30 \\
.37\end{array}$ & $\begin{array}{l}.09 \\
.18 \\
.28 \\
.37 \\
.46\end{array}$ & $\begin{array}{l}.11 \\
.22 \\
.33 \\
.44 \\
.56\end{array}$ & $\begin{array}{l}.13 \\
.26 \\
.39 \\
.52 \\
.65\end{array}$ & $\begin{array}{l}.15 \\
.30 \\
.44 \\
.59 \\
.74\end{array}$ & $\begin{array}{l}.17 \\
.33 \\
.50 \\
.67 \\
.83\end{array}$ & $\begin{array}{l}.18 \\
.37 \\
.56 \\
.74 \\
.93\end{array}$ & $\begin{array}{r}.20 \\
.41 \\
.61 \\
.81 \\
1.02\end{array}$ & $\begin{array}{r}.22 \\
.44 \\
.67 \\
.89 \\
1.11\end{array}$ & $\begin{array}{r}.24 \\
.48 \\
.72 \\
.96 \\
1.20\end{array}$ & $\begin{array}{r}.26 \\
.52 \\
.78 \\
1.04 \\
1.30\end{array}$ & $\begin{array}{r}.28 \\
.56 \\
.83 \\
1.11 \\
1.39\end{array}$ \\
\hline $\begin{array}{r}6 \\
7 \\
8 \\
10\end{array}$ & $\begin{array}{l}.11 \\
.19 \\
.15 \\
.17 \\
.18\end{array}$ & $\begin{array}{l}.22 \\
.26 \\
.30 \\
.33 \\
.37\end{array}$ & $\begin{array}{l}.33 \\
.39 \\
.44 \\
.50 \\
.56\end{array}$ & $\begin{array}{l}.44 \\
.52 \\
.59 \\
.67 \\
.84\end{array}$ & $\begin{array}{l}.56 \\
.65 \\
.74 \\
.83 \\
.93\end{array}$ & $\begin{array}{r}.67 \\
.78 \\
.89 \\
1.00 \\
1.11\end{array}$ & $\begin{array}{r}.78 \\
.91 \\
1.04 \\
1.17 \\
1.30\end{array}$ & $\begin{array}{r}.89 \\
1.04 \\
1.19 \\
1.33 \\
1.48\end{array}$ & $\begin{array}{l}1.00 \\
1.16 \\
1.33 \\
1.50 \\
1.67\end{array}$ & $\begin{array}{l}1.11 \\
1.30 \\
1.48 \\
1.67 \\
1.85\end{array}$ & $\begin{array}{l}1.22 \\
1.42 \\
1.63 \\
1.83 \\
2.04\end{array}$ & $\begin{array}{l}\text { 1. } 33 \\
1.55 \\
1.78 \\
\text { 2. } 00 \\
2.22\end{array}$ & $\begin{array}{l}1.44 \\
1.68 \\
1.92 \\
2.17 \\
2.41\end{array}$ & $\begin{array}{l}1.55 \\
1.81 \\
2.08 \\
2.33 \\
2.59\end{array}$ & $\begin{array}{l}1.67 \\
1.94 \\
2.22 \\
2.50 \\
2.78\end{array}$ \\
\hline $\begin{array}{l}11 \\
12 \\
13 \\
14 \\
15 .\end{array}$ & $\begin{array}{l}.20 \\
.22 \\
.24 \\
.26 \\
.28\end{array}$ & $\begin{array}{l}.41 \\
.44 \\
.48 \\
.52 \\
.56\end{array}$ & $\begin{array}{l}.61 \\
.67 \\
.72 \\
.78 \\
.83\end{array}$ & $\begin{array}{r}.82 \\
.89 \\
.96 \\
1.04 \\
1.11\end{array}$ & $\begin{array}{l}1.02 \\
1.11 \\
1.20 \\
1.30 \\
1.39\end{array}$ & $\begin{array}{l}1.22 \\
1.33 \\
1.44 \\
1.55 \\
1.67\end{array}$ & $\begin{array}{l}1.43 \\
1.56 \\
1.68 \\
1.81 \\
1.94\end{array}$ & $\begin{array}{l}1.63 \\
1.78 \\
1.92 \\
2.08 \\
2.22\end{array}$ & $\begin{array}{l}1.83 \\
2.00 \\
2.16 \\
2.33 \\
2.50\end{array}$ & $\begin{array}{l}2.04 \\
2.22 \\
2.41 \\
2.59 \\
2.78\end{array}$ & $\begin{array}{l}2.24 \\
2.44 \\
2.65 \\
2.85 \\
3.06\end{array}$ & $\begin{array}{l}\text { 2. } 44 \\
2.67 \\
2.89 \\
3.11 \\
3.33\end{array}$ & $\begin{array}{l}2.65 \\
2.89 \\
3.13 \\
3.37 \\
3.61\end{array}$ & $\begin{array}{l}2.85 \\
3.11 \\
3.37 \\
3.63 \\
3.89\end{array}$ & $\begin{array}{l}3.06 \\
3.33 \\
3.61 \\
3.89 \\
4.17\end{array}$ \\
\hline $\begin{array}{l}16 \\
17 \\
18 \\
19 \\
20\end{array}$ & $\begin{array}{l}.30 \\
.31 \\
.33 \\
.35 \\
.37\end{array}$ & $\begin{array}{l}.59 \\
.63 \\
.67 \\
.70 \\
.74\end{array}$ & $\begin{array}{r}.89 \\
.94 \\
1.00 \\
1.06 \\
1.11\end{array}$ & $\begin{array}{l}1.18 \\
1.26 \\
1.33 \\
1.41 \\
1.48\end{array}$ & $\begin{array}{l}1.48 \\
1.57 \\
1.67 \\
1.76 \\
1.85\end{array}$ & $\begin{array}{l}1.78 \\
1.89 \\
2.00 \\
2.11 \\
2.22\end{array}$ & $\begin{array}{l}2.07 \\
2.20 \\
2.33 \\
2.46 \\
2.59\end{array}$ & $\begin{array}{l}2.37 \\
2.52 \\
2.67 \\
2.82 \\
2.96\end{array}$ & $\begin{array}{l}2.67 \\
2.83 \\
3.00 \\
3.17 \\
3.33\end{array}$ & $\begin{array}{l}2.95 \\
3.15 \\
3.33 \\
3.52 \\
3.70\end{array}$ & $\begin{array}{l}3.26 \\
3.46 \\
3.67 \\
3.87 \\
4.07\end{array}$ & $\begin{array}{l}3.56 \\
3.78 \\
4.00 \\
4.22 \\
4.44\end{array}$ & $\begin{array}{l}3.85 \\
4.09 \\
4.33 \\
4.57 \\
4.81\end{array}$ & $\begin{array}{l}4.15 \\
4.41 \\
4.67 \\
4.92 \\
5.18\end{array}$ & $\begin{array}{l}4.44 \\
4.72 \\
5.00 \\
5.28 \\
5.56\end{array}$ \\
\hline $\begin{array}{l}21 \\
22 \\
23 \\
24 \\
24 \\
25\end{array}$ & $\begin{array}{l}.39 \\
.41 \\
.43 \\
.44 \\
.46\end{array}$ & $\begin{array}{l}.78 \\
.81 \\
.85 \\
.89 \\
.92\end{array}$ & $\begin{array}{l}1.17 \\
1.22 \\
1.28 \\
1.33 \\
1.39\end{array}$ & $\begin{array}{l}1.55 \\
1.63 \\
1.70 \\
1.78 \\
1.85\end{array}$ & $\begin{array}{l}1.94 \\
2.04 \\
2.13 \\
2.22 \\
2.31\end{array}$ & $\begin{array}{l}2.33 \\
2.44 \\
2.56 \\
2.67 \\
2.78\end{array}$ & $\left\{\begin{array}{l}2.72 \\
2.85 \\
2.98 \\
3.11 \\
3.24\end{array}\right.$ & $\begin{array}{l}3.11 \\
3.26 \\
3.41 \\
3.56 \\
3.70\end{array}$ & $\begin{array}{l}3.50 \\
3.67 \\
3.83 \\
4.00 \\
4.17\end{array}$ & $\begin{array}{l}3.89 \\
4.07 \\
4.26 \\
4.44 \\
4.63\end{array}$ & $\begin{array}{l}4.28 \\
4.48 \\
4.68 \\
4.89 \\
5.09\end{array}$ & $\begin{array}{l}4.67 \\
4.89 \\
5.11 \\
5.33 \\
5.56\end{array}$ & $\begin{array}{l}5.06 \\
5.30 \\
5.54 \\
5.78 \\
6.02\end{array}$ & $\begin{array}{l}5.44 \\
5.70 \\
5.96 \\
6.22 \\
6.48\end{array}$ & $\begin{array}{l}6.1 \\
6.3 \\
6.6 \\
6.9\end{array}$ \\
\hline $\begin{array}{l}26 \\
27 \\
28 \\
29 \\
89\end{array}$ & $\begin{array}{l}.48 \\
.50 \\
.52 \\
.54 \\
.56\end{array}$ & $\begin{array}{r}.96 \\
1.00 \\
1.04 \\
1.07 \\
1.11\end{array}$ & $\begin{array}{l}1.44 \\
1.50 \\
1.55 \\
1.61 \\
1.67\end{array}$ & $\begin{array}{l}1.92 \\
2.00 \\
2.07 \\
2.15 \\
2.22\end{array}$ & $\begin{array}{l}2.41 \\
2.50 \\
2.59 \\
2.68 \\
2.78\end{array}$ & $\begin{array}{l}2.89 \\
3.00 \\
3.11 \\
3.22 \\
3.33\end{array}$ & $\begin{array}{l}3.37 \\
3.50 \\
3.63 \\
3.76 \\
3.89\end{array}$ & $\begin{array}{l}3.85 \\
4.00 \\
4.15 \\
4.30 \\
4.44\end{array}$ & $\begin{array}{l}4.33 \\
4.50 \\
4.67 \\
4.83 \\
5.00\end{array}$ & $\begin{array}{l}4.82 \\
5.00 \\
5.18 \\
5.37 \\
5.55\end{array}$ & $\begin{array}{l}5.30 \\
5.50 \\
5.70 \\
5.91 \\
6.11\end{array}$ & $\begin{array}{l}5.78 \\
6.00 \\
6.22 \\
6.44 \\
6.67\end{array}$ & $\begin{array}{l}6.26 \\
6.50 \\
6.74 \\
6.98 \\
7.22\end{array}$ & $\begin{array}{l}6.74 \\
7.00 \\
7.26 \\
7.52 \\
7.78\end{array}$ & $\begin{array}{l}7.50 \\
7.78 \\
8.06 \\
8.30\end{array}$ \\
\hline $\begin{array}{l}31 \\
32 \\
33 \\
34 \\
35\end{array}$ & $\begin{array}{l}.57 \\
.59 \\
.61 \\
.63 \\
.65\end{array}$ & $\begin{array}{l}1.15 \\
1.18 \\
1.22 \\
1.26 \\
1.30\end{array}$ & $\begin{array}{l}1.72 \\
1.78 \\
1.83 \\
1.89 \\
1.94\end{array}$ & $\begin{array}{l}2.30 \\
2.37 \\
2.44 \\
2.52 \\
2.59\end{array}$ & $\begin{array}{l}2.87 \\
2.96 \\
3.05 \\
3.15 \\
3.24\end{array}$ & $\begin{array}{l}3.44 \\
3.56 \\
3.67 \\
3.78 \\
3.89\end{array}$ & $\begin{array}{l}4.02 \\
4.15 \\
4.28 \\
4.40 \\
4.53\end{array}$ & $\begin{array}{l}4.59 \\
4.74 \\
4.89 \\
5.04 \\
5.18\end{array}$ & $\begin{array}{l}5.17 \\
5.33 \\
5.50 \\
5.67 \\
5.83\end{array}$ & $\begin{array}{l}5.74 \\
5.92 \\
6.11 \\
6.29 \\
6.48\end{array}$ & $\begin{array}{l}6.32 \\
6.52 \\
6.72 \\
6.93 \\
7.13\end{array}$ & $\begin{array}{l}6.89 \\
7.11 \\
7.33 \\
7.56 \\
7.78\end{array}$ & $\begin{array}{l}7.46 \\
7.70 \\
7.94 \\
8.18 \\
8.42\end{array}$ & $\begin{array}{l}8.04 \\
8.30 \\
8.55 \\
8.81 \\
9.08\end{array}$ & $\begin{array}{l}8.8 \\
9.1 \\
9.4 \\
9.7\end{array}$ \\
\hline $\begin{array}{l}36 \\
87 \\
38 \\
\$ 99 \\
40\end{array}$ & $\begin{array}{l}.67 \\
.68 \\
.70 \\
.72 \\
.74\end{array}$ & $\begin{array}{l}1.33 \\
1.37 \\
1.41 \\
1.44 \\
1,48\end{array}$ & $\begin{array}{l}2.00 \\
2.06 \\
2.11 \\
2.17 \\
2.32\end{array}$ & $\begin{array}{l}2.67 \\
2.74 \\
2.82 \\
2.89 \\
2.96\end{array}$ & $\begin{array}{l}3.33 \\
3.42 \\
3.52 \\
3.61 \\
3.70\end{array}$ & $\begin{array}{l}4.00 \\
4.11 \\
4.22 \\
4.33 \\
4.44\end{array}$ & $\begin{array}{l}4.65 \\
4.79 \\
4.92 \\
5.05 \\
5.18\end{array}$ & $\begin{array}{l}5.33 \\
5.48 \\
5.63 \\
5.78 \\
5.92\end{array}$ & $\begin{array}{l}6.00 \\
6.17 \\
6.33 \\
6.50 \\
6.67\end{array}$ & $\begin{array}{l}6.67 \\
6.85 \\
7.03 \\
7.22 \\
7.41\end{array}$ & $\left\{\begin{array}{l}7.33 \\
7.54 \\
7.74 \\
7.95 \\
8.15\end{array}\right.$ & $\begin{array}{l}8.00 \\
8.22 \\
8.44 \\
8.67 \\
8.89\end{array}$ & $\begin{array}{l}8.67 \\
8.91 \\
9.15 \\
9.39 \\
9.63\end{array}$ & $\begin{array}{r}9.33 \\
9.59 \\
9.35 \\
10.11 \\
10.37\end{array}$ & $\begin{array}{l}10.28 \\
10.56 \\
10.89 \\
11.11\end{array}$ \\
\hline
\end{tabular}

Table $\mathrm{X}$ gives the number of cubic yards in a TRTANGLE 1 foot deep for a given wilth and height. CAUT1ON: Values obtained from the ahove tables are for only the one TRIANGLE of the cross-section under consideration.

Currections for tenths of feet in width and height may be made by considering the numbers on the table from 1 to 9 ss tenths and taking one tenth the values found in the tables. For example, to find the number of cubic yards when width $=\mathbb{W}=16.2$ and Height $=\mathrm{H}=\mathbf{5 . 3}$, opposite 16 in "Width" column and under 5 in the "Height" coluinn read 1.48. To correct for additional 0.2 foot of wid th, opposite 2 in the "Width" column and under 5 in the "Height" column read $0.1 \mathrm{~s}$ and correct to 018 . To correct for additional 0.3 foot in height, under 3 in "Height" column and opposite 16 in "Width" column read 0.89 and correct to .059 . Therefore, the total cubic yards in the given TRIANGLE for a depth of 1 foot $=1.4 S+.01 S+.089=1.587$ or approximately si cu. $y \mathrm{ds}$. per 100 feet.

If width exceeds $\$ 0$ feet, use one half real width and multiply resuit by 2 . If hoth width and heiglit are larger than values given on table use one half of each value and multiply result hy 4 . 




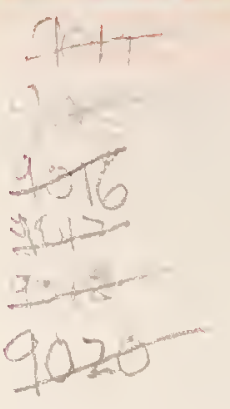


